\title{
Where genotype is not predictive of phenotype: towards an understanding of the molecular basis of reduced penetrance in human inherited disease
}

\author{
David N. Cooper • Michael Krawczak • \\ Constantin Polychronakos • Chris Tyler-Smith • \\ Hildegard Kehrer-Sawatzki
}

Received: 3 May 2013/Accepted: 15 June 2013/Published online: 3 July 2013

(C) The Author(s) 2013. This article is published with open access at Springerlink.com

\begin{abstract}
Some individuals with a particular diseasecausing mutation or genotype fail to express most if not all features of the disease in question, a phenomenon that is known as 'reduced (or incomplete) penetrance'. Reduced penetrance is not uncommon; indeed, there are many known examples of 'disease-causing mutations' that fail to cause disease in at least a proportion of the individuals who carry them. Reduced penetrance may therefore explain not only why genetic diseases are occasionally transmitted through unaffected parents, but also why healthy individuals can harbour quite large numbers of potentially disadvantageous variants in their genomes without suffering any obvious ill effects. Reduced penetrance can be a function of the specific mutation(s) involved or of allele dosage.
\end{abstract}

D. N. Cooper $(\bowtie)$

Institute of Medical Genetics, School of Medicine, Cardiff University, Heath Park, Cardiff CF14 4XN, UK

e-mail: cooperDN@cardiff.ac.uk

M. Krawczak

Institute of Medical Informatics and Statistics, Christian-Albrechts University, 24105 Kiel, Germany

e-mail:krawczak@medinfo.uni-kiel.de

C. Polychronakos

Department of Pediatrics/Human Genetics,

McGill University Health Center, Montreal, Canada

e-mail: constantin.polychronakos@mcgill.ca

C. Tyler-Smith

The Wellcome Trust Sanger Institute, Wellcome Trust Genome Campus, Hinxton, Cambridge CB10 1SA, UK

e-mail: cts@sanger.ac.uk

H. Kehrer-Sawatzki

Institute of Human Genetics, University of Ulm,

Albert-Einstein-Allee 11, $89081 \mathrm{Ulm}$, Germany

e-mail: hildegard.kehrer-sawatzki@uni-ulm.de
It may also result from differential allelic expression, copy number variation or the modulating influence of additional genetic variants in cis or in trans. The penetrance of some pathogenic genotypes is known to be age- and/or sexdependent. Variable penetrance may also reflect the action of unlinked modifier genes, epigenetic changes or environmental factors. At least in some cases, complete penetrance appears to require the presence of one or more genetic variants at other loci. In this review, we summarize the evidence for reduced penetrance being a widespread phenomenon in human genetics and explore some of the molecular mechanisms that may help to explain this enigmatic characteristic of human inherited disease.

\section{Introduction}

One old conundrum in human genetics is that not everyone with a given pathological mutation (or mutations) will eventually develop the disease in question. The proportion of those individuals harbouring a particular pathogenic mutation or genotype who exhibit clinical signs of the associated disorder within a specific and clearly defined time period is termed the penetrance of that disorder. If this proportion equals $100 \%$, the disease and/or disease genotype(s) are said to show complete penetrance. If not, they are said to exhibit reduced (or incomplete) penetrance. Reduced penetrance is likely to be a consequence of the combination of a variety of different genetic and environmental factors. A classic example is phenylketonuria, where inactivating mutations in the $P A H$ gene encoding the enzyme phenylalanine hydroxylase lead to severe intellectual disability in the context of a normal diet, whereas a life-long phenylalanine-restricted diet makes possible a relatively healthy life (Blau et al. 2010). Regrettably, few 
other examples of reduced penetrance are as simple, well understood or clinically manipulable as this one, and the precise mechanisms by which different factors give rise to reduced penetrance remain largely unknown (Zlotogora 2003).

In formal terms, penetrance measures the proportion of individuals in a given population with a specific diseaseassociated genotype who also express the corresponding disease phenotype. Large family/population studies have traditionally been considered necessary to measure penetrance, either for specific mutations/genotypes or as a disease average. 'Cascade genetic screening', whereby relatives of previously identified carriers are screened both for the mutation(s) in question and for the presence/ absence of clinical symptoms, is one means to determine the degree of penetrance for a given genotype (Berge et al. 2008). As we discuss below, large-scale sequencing and genotyping studies of apparently healthy individuals from the general population provide a powerful new approach to understanding the penetrance of pathological mutations/ genotypes. The outcomes of such studies should allow us to predict how likely it is that a given disease will manifest itself in an individual who carries a specific genotype.

Reduced penetrance is most obviously evident in disorders that follow an autosomal dominant mode of inheritance. In these instances, reduced penetrance is a characteristic of the underlying mutation, rather than a genotype. However, reduced penetrance can also occur in autosomal recessive disorders where one and the same mutation can have different phenotypic effects, depending at least in part upon the second disease allele present. Irrespective of the mode of inheritance, in most cases penetrance is likely to be a function of the specific mutation(s) involved. Thus, in some conditions normally characterized by an autosomal dominant mode of inheritance, two incompletely penetrant (or otherwise non-penetrant) alleles may act in recessive fashion while mimicking the normal dominant form of the disease (e.g. Grundy et al. 1991; Croxen et al. 2002; Kowalewski et al. 2007; Castaman et al. 2007; Rossetti et al. 2009; Vujic et al. 2010; Schaaf et al. 2011a). For a dominantly inherited condition, one consequence of reduced penetrance is that the clinical phenotype may not be evident in one generation, but can nevertheless still be transmitted (through an apparently unaffected parent) to subsequent generations where it again manifests itself; specimen examples of this from clinical practice include hereditary hyperekplexia (Kwok et al. 2001), cherubism (Preda et al. 2010), retinitis pigmentosa (Rio Frio et al. 2009), rhabdoid tumour predisposition syndrome (Ammerlaan et al. 2008), autism spectrum disorder (Fujita-Jimbo et al. 2012) and hypercholesterolaemia (Garcia-Garcia et al. 2011). For all the above reasons, reduced penetrance presents a major challenge to genetic counsellors attempting to interpret the medical history of a patient's family to quantify the disease risk to the patient's offspring (Emery 1986; Otto and Maestrelli 2000).

Reduced penetrance is not uncommon; indeed, there are many known examples of bona fide disease-causing variants or genotypes that fail to cause disease in at least a proportion of individuals who carry them (Zlotogora 2003; Waalen and Beutler 2009). By definition, penetrance refers to the black and white issue of whether the clinical phenotype associated with a certain genotype is present or not. We routinely distinguish it from variable expressivity which refers to the degree of variation of the clinical phenotype in those individuals with a particular genotype. Although, in principle, penetrance and expressivity are distinct terms with specific meanings (depending upon the way a given clinical phenotype is defined), in practice they are closely inter-related and likely to manifest via similar mechanisms. We also distinguish reduced penetrance from small effect size. Thus, most carriers of the risk alleles discovered by genome-wide association studies (GWAS) may never develop the disease in question; this is because these variants generally only make a small contribution to the multifactorial aetiology of the condition. To be able instead to say that the variant is non-penetrant in some individuals, we require the same variant in other individual(s) to make the crucial difference between the phenotype being manifested or not. In what follows, we shall focus specifically on the molecular mechanisms that could account for the phenomenon of reduced penetrance. This notwithstanding, the discussion of genetic variants that modulate the expressivity of a particular disease has sometimes also been deemed appropriate.

In this review, we present the evidence for reduced penetrance being a widespread phenomenon in human genetics, evidence that comes not only from a plethora of case studies of monogenic disorders but also more recently from the next generation sequencing of entire exomes or genomes of apparently normal healthy individuals from the general population. Here, we have attempted to explore the individual genetic components that contribute to the complexity of primarily monogenic disorders with reduced penetrance. This notwithstanding, the action of modifier genes is one of the mechanisms responsible for reduced penetrance, and one that has increasingly become recognized as blurring the distinction between monogenic conditions and complex disease (Nadeau 2003; Badano and Katsanis 2003; Sidransky 2006). Finally, we explore some of the molecular mechanisms which could account for the reduced penetrance of many human inherited disorders and provide evidence to support the view that, at least in some instances, complete penetrance requires the presence of one or more genetic variants at other loci. 


\section{Deleterious and disease alleles in the general population}

In the wake of the sequencing of multiple human genomes, it has become apparent that healthy individuals can harbour quite large numbers of potentially disadvantageous variants without suffering any obvious ill effects (The 1000 Genomes Project Consortium 2010; MacArthur et al. 2012; Xue et al. 2012; Shen et al. 2013a). The underlying reasons are likely to be many and varied: thus, the variants may damage the protein in question but the intact protein may not be necessary for the health of the carrier; individuals may be asymptomatic carriers of single-mutant alleles that could, in homozygosity or compound heterozygosity, cause recessive disease; the mutation may be dominant but the clinical phenotype might only be mild and classed as lying within the range of normal healthy variation; the disorder might be late in onset with expression being age- or sexdependent; or the disorder may require additional genetic and/or environmental factors for it to manifest clinically.

Assessing the magnitude of the 'genetic burden' imposed on the general population by the presence of deleterious alleles has been a key aim of medical and population genetics for many decades. With the advent of large-scale sequencing technologies, it has become possible to estimate the number of amino acid substitutions in the human exome that would be predicted in silico to be damaging (Kryukov et al. 2007; Lohmueller et al. 2008; Boyko et al. 2008; Goode et al. 2010). However, personal genome sequences have not only provided estimates of the number of disease variants carried by each subject (Asan et al. 2011), but have also given us a glimpse of the likely complexity of the functional interpretation of such data (Ashley et al. 2010; Strom and Gorin 2013). More recently, Tennessen et al. (2012) suggested that $2.3 \%$ of the 13,595 single nucleotide variants carried by the average person impact upon protein function, involving $\sim 313$ genes per human genome. Adopting an alternative approach, Bell et al. (2011) surveyed 437 genes known to be related to a recessive Mendelian disease and identified 2.8 mutations per individual (range 0-7). Taken together, these studies suggest that individuals typically carry hundreds of mildly disadvantageous variants and perhaps several tens of potentially severe disease alleles.

In a pilot study for the 1000 Genomes Project Consortium (2010), we reported the prevalence of disease alleles, defined by reference to the disease-causing mutations (DMs) listed in the Human Gene Mutation Database (HGMD; http://www.hgmd.org; Stenson et al. 2009), in population samples of African, European and East Asian origin. These numbers were surprisingly high: $57-80$ disease alleles per individual in a sample of 179 participants. Moreover, further examination of these data showed that 191 disease alleles were present in the homozygous state in at least one individual, and hence were not simply present because their phenotypic/clinical effects had been masked by a normal allele. Although little phenotypic information other than sex, ethnicity, place of origin and relationship to other participants is available for the 1000 Genomes Project donors, the Project's ethical framework requires that sample donors are non-vulnerable adults who are competent to consent to participation in the project and hence are likely to lack any obvious severe disease phenotype. Instead, we need to seek some other explanation for the high number of DMs present. One possibility is that the penetrance of disease alleles and genotypes could be much lower and more variable than previously realized. Conventionally, most studies of human inherited disease that have contributed mutation data to HGMD have sought to identify a disease genotype, given a clinical phenotype (Cooper et al. 2010). This is a very different scenario from identifying a phenotype given a (potentially disease associated) genotype. Indeed, the former strategy inevitably avoids the whole issue of penetrance because, by definition, it focuses exclusively upon those individuals in whom the mutation of interest has been penetrant. It follows that a given variant could be genuinely causative in a set of individuals manifesting a particular disease, yet may also be present in a set of healthy individuals who differ in any one of a number of ways to be discussed below.

Since the 1000 Genomes Project Consortium paper was published in 2010, two further reports have appeared that served to improve our knowledge of deleterious mutations in the genomes of apparently healthy individuals. The most readily recognized deleterious variants in the human genome are those that disrupt a protein-coding gene, either by leading to a loss of function (e.g. a nonsense or frameshift variant) or by altering an amino acid in the encoded protein (missense variants). The former category of mutation has been studied by MacArthur et al. (2012) who identified 1,285 putative loss-of-function variants (i.e. nonsense mutations, splice site-disrupting single nucleotide variants, micro-insertions/micro-deletions, etc.) in the genome sequences of 185 humans from the 1000 Genomes Project. From these data, they estimated that an average human genome typically contains $\sim 100$ genuine loss-of-function variants with $\sim 20$ genes having both copies inactivated. Following on from this study, Xue et al. (2012) focused on missense mutations, ascertaining the numbers of potentially deleterious missense variants in the genomes of apparently healthy individuals using low-coverage wholegenome sequence data from 179 individuals in the 1000 Genomes Pilot Project. Each individual was found to carry 281-515 missense substitutions predicted with a high degree of confidence to be damaging to the gene product, 40-85 of which were present in the homozygous state. Taken together, these studies suggest that a typical healthy 
individual has about 80 of their genes severely damaged or inactivated in both copies, further emphasizing the stark contrast between damage to gene and protein on the one hand, and damage to health on the other. The 1000 Genomes Project participants also carried 40-110 variants (3-24 homozygous) classified by HGMD as DMs. Whereas many of these DMs could conceivably represent disease attribution errors of some kind, between 0 and 8 DMs per individual ( $0-1$ homozygous) were predicted to be highly damaging.

Among the missense DMs, Xue et al. (2012) identified known pathological variants such as $H B B \quad$ (c.20A $>\mathrm{T}$; p.Glu7Val), which leads to increased resistance to malaria in heterozygotes but to sickle cell disease in homozygotes [confined to Africans (Yoruba, YRI) in whom there were
12 heterozygotes and 1 homozygote]. In addition, Xue et al. (2012) identified an USH2A variant (c.2138G $>$ C; p.Gly713Arg), previously reported as being causal for Usher syndrome type 2 , a recessive disorder characterized by combined deafness and blindness; three homozygotes were noted in the YRI. Manual curation of the HGMD1000GP overlap revealed the presence of three types of DM: (1) plausible severe disease-causing variants, (2) variants convincingly causative for pathological conditions, yet quite compatible with adult life and (3) variants probably incorrectly assigned as disease causing. After applying various filtering criteria designed to enrich for true disease alleles, the list was reduced to 45 candidates (Xue et al. 2012). Of these putative disease alleles, 34 were present in the heterozygous state and were deemed likely to

Table 1 Disease variants potentially capable of either causing dominant disease, or causing recessive disease and observed in the homozygous state, detected in 1000 Genomes Project participants (data from Xue et al. 2012)

\begin{tabular}{|c|c|c|c|c|c|c|c|}
\hline $\begin{array}{l}\text { Disease (MIM } \\
\text { number) }\end{array}$ & Inheritance & Gene & $\begin{array}{l}\text { HGVS cDNA } \\
\text { mutation }\end{array}$ & $\begin{array}{l}\text { Protein } \\
\text { alteration }\end{array}$ & $\begin{array}{l}\text { Total } \\
\text { homozygotes }\end{array}$ & $\begin{array}{l}\text { Total } \\
\text { heterozygotes }\end{array}$ & Comments \\
\hline $\begin{array}{l}\text { Ataxia } \\
\text { telangiectasia } \\
\text { (MIM\# 607585) }\end{array}$ & $\mathrm{AR}$ & ATM & $\begin{array}{l}\text { NM_000051.3: } \\
\text { c. } 4258 \mathrm{C}>\mathrm{T}\end{array}$ & p.Leu1420Phe & 1 & 3 & $\begin{array}{l}\text { Low-penetrance breast } \\
\text { cancer susceptibility } \\
\text { allele }\end{array}$ \\
\hline $\begin{array}{l}\text { Usher syndrome } \\
\text { type IIA (MIM\# } \\
\text { 276901) }\end{array}$ & AR & $U S H 2 A$ & $\begin{array}{l}\text { NM_206933.2: } \\
\text { c.2137G }>C\end{array}$ & p.Gly713Arg & 3 & 22 & $\begin{array}{l}\text { Probable complex } \\
\text { pathogenicity; neutral } \\
\text { in YRI? }\end{array}$ \\
\hline $\begin{array}{l}\text { Nephronophthisis } \\
4 \text { (MIM\# } \\
606966)\end{array}$ & $\mathrm{AR}$ & NPHP4 & $\begin{array}{l}\text { NM_015102.3: } \\
\text { c. } 2542 \mathrm{C}>\mathrm{T}\end{array}$ & p.Arg848Trp & 1 & 2 & $\begin{array}{l}\text { Growth retardation; } \\
\text { adult-onset renal } \\
\text { disease }\end{array}$ \\
\hline $\begin{array}{l}\text { Cushing } \\
\text { syndrome } \\
\text { (MIM\# 607397) }\end{array}$ & $\mathrm{AR}$ & $M C 2 R$ & $\begin{array}{l}\text { NM_000529.2: } \\
\text { c.833T }>\text { G }\end{array}$ & p.Phe278Cys & 1 & 10 & $\begin{array}{l}\text { Hormonal disorder; } \\
\text { variable gender-specific } \\
\text { symptoms; variant } \\
\text { functionally defective } \\
\text { in vitro }\end{array}$ \\
\hline $\begin{array}{l}\text { Low- } \\
\text { phospholipid- } \\
\text { associated } \\
\text { cholelithiasis } \\
\text { (MIM\# 171060) }\end{array}$ & $\mathrm{AR}$ & $A B C B 4$ & $\begin{array}{l}\text { NM_000443.3: } \\
\text { c. } 2363 \mathrm{G}>\mathrm{A}\end{array}$ & p.Arg788Gln & 2 & 9 & Adult onset \\
\hline $\begin{array}{l}\text { Cardiomyopathy, } \\
\text { hypertrophic } \\
\text { (MIM\# 115197) }\end{array}$ & $\mathrm{AD}$ & МYВРC3 & $\begin{array}{l}\text { NM_000256.3: } \\
\text { c.1519G>A }\end{array}$ & p.Gly507Arg & 0 & 2 & $\begin{array}{l}\text { Late onset; incomplete } \\
\text { penetrance }\end{array}$ \\
\hline $\begin{array}{l}\text { Glaucoma, } \\
\text { primary open } \\
\text { angle (MIM\# } \\
609887 \text { ) }\end{array}$ & $\mathrm{AD}$ & WDR36 & $\begin{array}{l}\text { NM_139281.2: } \\
\text { c.1586G }>A\end{array}$ & p.Arg529Gln & 0 & 1 & $\begin{array}{l}\text { Adult onset; variant } \\
\text { functionally defective } \\
\text { in vitro }\end{array}$ \\
\hline $\begin{array}{l}\text { Colorectal cancer, } \\
\text { nonpolyposis } \\
\text { (MIM\# 609310) }\end{array}$ & $\mathrm{AD}$ & $M L H 1$ & $\begin{array}{l}\text { NM_000249.3: } \\
\text { c. } 1742 \mathrm{C}>\mathrm{T}\end{array}$ & p.Pro581Leu & 0 & 1 & $\begin{array}{l}\text { Adult onset; variant } \\
\text { functionally defective } \\
\text { in vitro }\end{array}$ \\
\hline $\begin{array}{l}\text { Renal cell } \\
\text { carcinoma } \\
\text { (MIM\# 144700) }\end{array}$ & $\mathrm{AD}$ & $F L C N$ & $\begin{array}{l}\text { NM_144997.5: } \\
\text { c. } 715 \mathrm{C}>\mathrm{T}\end{array}$ & p.Arg239Cys & 0 & 1 & Late onset \\
\hline $\begin{array}{l}\text { Heparin cofactor } \\
2 \text { deficiency } \\
\text { (MIM\# 612356) }\end{array}$ & $\mathrm{AD}$ & SERPIND1 & $\begin{array}{l}\text { NM_000185.3: } \\
\text { c.623G }>A\end{array}$ & p.Arg208His & 0 & 2 & $\begin{array}{l}\text { Deficiency state, but no } \\
\text { overt disease; risk factor } \\
\text { for thrombophilia }\end{array}$ \\
\hline $\begin{array}{l}\text { Lp(a) deficiency } \\
\text { (MIM\# 152200) }\end{array}$ & $\mathrm{AD}$ & $L P A$ & $\begin{array}{l}\text { NM_005577.2: } \\
\text { c. } 4289+1 \mathrm{G}>\mathrm{A}\end{array}$ & $\begin{array}{l}\text { essential } \\
\text { splice site }\end{array}$ & 0 & 5 & $\begin{array}{l}\text { Late onset; risk factor for } \\
\text { heart disease }\end{array}$ \\
\hline
\end{tabular}


be present in asymptomatic carriers. Among the remaining 11 (Table 1), the 6 linked to dominant disorders were explicable in terms of either late onset (e.g. Gly507Arg in MYBPC3) or covert disease (Arg208His in SERPIND1). In similar vein, the presence of homozygotes for four of the five recessive disorders could be explicable in terms of late onset and/or reduced penetrance of disease. The USH2A mutation (Gly713Arg) was, however, intriguing: this variant was predicted to be damaging to the protein, and pathogenic in some populations but not in others (e.g. YRI). One explanation put forward to explain this apparent contradiction was that, in the YRI population, the USH2A locus is subject to copy number variation (Matsuzaki et al. 2009) that could provide functional complementation of the mutant gene. In the majority of cases, however, the most likely explanation for the absence of disease at the time of recruitment was considered to be the probable late onset of disease, although clinical penetrance was often variable, and some phenotypes, such as loose anagen hair syndrome [caused by Glu337Lys in KRT75 (MIM 600628)], might not even be regarded as "diseases" sensu stricto. These factors notwithstanding, the findings of Xue et al. (2012) suggest that incidental findings which are potentially relevant to health and well-being might be made in as many as $11 \%$ of individuals sequenced.

Reduced penetrance is one of several possible explanations for why some variants of putative pathological significance, listed in HGMD and/or Locus-specific Mutation Databases, nevertheless occur in apparently healthy individuals (Ashley et al. 2010; Bell et al. 2011; Xue et al. 2012; Golbus et al. 2012; Wang et al. 2013a; Kenna et al. 2013; Shen et al. 2013a). It is not hard to see why reduced penetrance might be much more common among described mutations than originally thought: whereas known pathological mutations have almost invariably been identified through retrospective analyses of families or well-defined groups of clinically symptomatic patients, relatively few prospective studies of asymptomatic carriers have so far been performed to derive estimates of penetrance (e.g. Jensen et al. 2013; Mavaddat et al. 2013). Indeed, establishing that a specific mutation identified in a particular patient with a given disease is the pathological lesion responsible for that individual's clinical phenotype does not allow one automatically to judge whether this mutant genotype will invariably give rise to the same clinical phenotype in all other individuals harbouring it. This can only be established (or refuted) empirically by comprehensive, ideally prospective, studies of the genotype in question.

One fairly obvious reason why comparing the output of genome sequencing projects (e.g. the 1000 Genomes Project) with a comprehensive database of putatively pathological mutations (e.g. HGMD) is likely to generate a considerable number of potentially pathogenic mutations in the general population, is that many such mutations are quite frequent in the population at large. In particular, carrier frequencies for mutations underlying recessive conditions can often be quite high. Thus, the $A B C A 4$ Gly863Ala mutation causing Stargardt disease has a carrier frequency of $1.8 \%$ in Europe (Maugeri et al. 2002), the GJB2 35delG mutation causing congenital deafness has a carrier frequency of $2.9 \%$ in southern Europe (Gasparini et al. 2000), the ERCC 8 Tyr322Term mutation causing Cockayne syndrome has a carrier frequency of $6.8 \%$ in Israeli Christian Arabs (Khayat et al. 2010) and the SPG7 Ala510Val mutation associated with adult-onset neurogenetic disease has a carrier frequency of 3-4\% in the UK population (Roxburgh et al. 2013). Disease allele frequencies can be as high as $10 \%$ in certain ethnic groups, e.g. Jews (Zlotogora et al. 2007; Ostrer and Skorecki 2013). A recent screen of the Korean population for 20 common mutations contributing to six autosomal recessive disorders yielded a combined carrier frequency of $6.7 \%$ (Song et al. 2012). Screening an ethnically diverse US population sample $(N=364,890)$ for 87 different $C F T R$ mutations responsible for causing cystic fibrosis yielded a combined carrier frequency of $2.6 \%$ (Rohlfs et al. 2011). As part of the NHLBI-Go Exome Sequencing Project, a screen of 5,400 individuals from the general population for variants in eight long QT syndrome genes yielded a total of 33 different missense mutations (affecting 173 alleles), representing a carrier frequency of $3.2 \%$ (Refsgaard et al. 2012). A similar screen for cardiomyopathy-associated gene variants yielded combined carrier frequencies for mutations reported to be disease associated of $25 \%(1,474 / 5,810)$ for hypertrophic cardiomyopathy, $15 \%(963 / 6,334)$ for dilated cardiomyopathy and (22\%) 1,393/6,359 for arrhythmogenic right ventricular cardiomyopathy (Andreasen et al. 2013); the high number of detected cardiomyopathy-associated gene variants suggests, however, that many are only modest disease modifiers or even non-pathogenic. Nishiguchi and Rivolta (2012) screened 46 complete genome sequences from the general population for mutations in 106 genes associated with recessively inherited retinal degeneration and identified null mutations in ten individuals (22\%). Finally, a routine screen of 23,453 individuals for 417 pathogenic mutations associated with a total of 108 recessive diseases concluded that $24 \%$ of individuals were carriers of at least one disorder, whilst $5.2 \%$ were carriers of two or more disorders (Lazarin et al. 2013). Clearly, there is a veritable abundance of actual and potential pathological variants segregating in the general population.

The examples that we cite in the text that follows and in the accompanying tables are by no means comprehensive and have been provided simply to illustrate the many and varied mechanisms that are already known to underlie the phenomenon of reduced penetrance in relation to clinical disorders. However, these examples also demonstrate that 
it is often very hard for authors, diagnosticians and mutation database curators alike to classify the pathogenicity of identified variants with any degree of certainty. Thus, is a given missense mutation a bona fide pathological lesion that exhibits greatly reduced penetrance, or is it essentially a neutral or near-neutral variant that occasionally finds itself in cis to other variants that are responsible for conferring the disease phenotype upon the individual concerned, or is it a variant with small effect that is sometimes present in affected individuals whose major causative lesions remain unknown?

\section{Incomplete penetrance in dominant and recessive conditions}

A considerable number of autosomal dominant disorders are characterized by incomplete penetrance. Well-studied examples include the hair disease monilethrix (KRT86; De Cruz et al. 2012), congenital cataract (GJA3; Burdon et al. 2004), different types of retinitis pigmentosa (PRPF8 and PRPF31; Maubaret et al. 2011; Saini et al. 2012), LMNA mutation-associated muscular phenotypes (Rankin et al. 2008) and long QT syndrome (Giudicessi and Ackerman 2013; Mathias et al. 2013).

One rather well-understood example of incomplete penetrance of a dominantly inherited mutation is factor V Leiden (F5, Arg534Gln; Arg506Gln in legacy nomenclature; rs6025) which occurs at polymorphic frequencies $(2-5 \%)$ in European populations, but is associated with a sixfold increased risk of venous thrombosis and a two- to threefold increased risk of pregnancy loss (Kujovich 2011). Despite these very evident disease associations, the vast majority of factor V Leiden carriers appear to be clinically unaffected. This could help to account for the high frequency of this variant in the general population, together perhaps with the survival advantage conferred by factor V Leiden carriership in various other clinical contexts including severe sepsis (Kerlin et al. 2003; van Mens et al. 2013). In similar vein, the asymptomatic (clinically covert) state is much more common than the clinically overt state in several other dominant disorders of haemostasis, including protein C deficiency (Tait et al. 1995; McColl et al. 1996), protein S deficiency (Dykes et al. 2001), antithrombin deficiency (Tait et al. 1994; McColl et al. 1996) and von Willebrand disease (Rodeghiero et al. 1987; Castaman et al. 2003). Thus, in disorders of haemostasis and thrombosis, even with well-characterized variants that are known to confer a significantly increased disease risk, the clinical penetrance is often so low that more healthy individuals carry the variant than those who actually manifest disease.

Reduced or incomplete penetrance has also been described for autosomal recessive disorders. Probably the best characterized example of incomplete penetrance in a recessive disorder is provided by the Cys282Tyr (rs1800562) mutation in the haemochromatosis (HFE) gene (Beutler 2003). The Tyr282 homozygous genotype is present in approximately 1 in 200 people of Northern European origin and is responsible for 80-90\% of hereditary haemochromatosis (Weiss 2010; Rochette et al. 2010). Although Tyr282 homozygosity displays a relatively high biochemical penetrance (i.e. iron accumulation), its clinical penetrance is low (McCune et al. 2006). Available data suggest that $38-50 \%$ of Tyr 282 homozygotes develop iron overload and 10-25\% develop some type of haemochromatosis-associated morbidity (Whitlock et al. 2006). However, these statistics conceal what appears to be a gender effect: large-scale studies of newly diagnosed Tyr 282 homozygotes, in whom liver disease had been specifically assessed, revealed that disease manifested in $24-43 \%$ of males, but only 1-14 \% of females (Rossi et al. 2008). Various genetic modifiers have been identified as influencing the clinical expression of haemochromatosis. These include mutations in the HAMP, HFE2 and TFR2 genes and polymorphisms in the BMP2, BMP4, CYBRD1, HP, LTA, MPO, TMPRSS6 and TNF genes (Milet et al. 2007; Rochette et al. 2010; Valenti et al. 2012; Pelucchi et al. 2012). In addition, several environmental modifiers (e.g. diet, alcohol intake) are also known to affect the penetrance of the $H F E$ genotype.

In another recessive disorder, Gaucher disease, the most common GBA mutation, Asn370Ser (Asn409Ser in HGVS nomenclature; rs76763715), is also characterized by low penetrance and exhibits extensive phenotype heterogeneity even in the homozygous state (Sibille et al. 1993; Horowitz et al. 1998; Fairley et al. 2008). However, close examination of asymptomatic Ser370 homozygotes, serendipitously diagnosed by prenatal carrier screening, revealed a variety of previously unidentified disease manifestations indicating that the clinical penetrance of this disease genotype may be greater than previously appreciated (Balwani et al. 2010).

\section{Influence of mutation type on penetrance}

Clinical penetrance is in part a function of the mutation(s) in question. For a given disease, some causal mutations may exhibit complete clinical penetrance, whereas other mutations in the same gene show incomplete or even very low penetrance. Thus, whereas the penetrance of the most common CFTR gene lesion, $\triangle$ Phe508 (rs113993960), in cystic fibrosis is very high, the penetrance of the CFTR Arg117His (rs78655421) mutation (in any allele combination) appears to be so low as to call into question its putative role as a pathological mutation (Thauvin-Robinet et al. 2009).

As yet, relatively few studies have been performed on low-penetrance mutations with a view to identifying the features responsible at the molecular level for their low 
penetrance. One exception is in retinoblastoma where it has been found that low-penetrance $R B 1$ mutations tend either to lead to a reduction in the amount of $\mathrm{Rb}$ protein produced (through promoter or splice site mutations) or yield a partially functional $\mathrm{Rb}$ molecule through missense mutation or in-frame deletion (Onadim et al. 1992; Kratzke et al. 1994; Bremner et al. 1997; Otterson et al. 1997; Scheffer et al. 2000; Genuardi et al. 2001; Harbour 2001; Klutz et al. 2002; Lefévre et al. 2002; Valverde et al. 2005; Sánchez-Sánchez et al. 2005; Sampieri et al. 2006; GámezPozo et al. 2007; Park et al. 2008; Hung et al. 2011). Of particular interest is the category of temperature-sensitive mutations in the $\mathrm{Rb}$ pocket domain $(\Delta \mathrm{Asn} 480$, Arg661Trp, Cys712Arg) whose 'reversible fluctuations' in a threshold level of $\mathrm{Rb}$ pocket-binding activity could be responsible for their characteristic low penetrance (Otterson et al. 1999). On the basis of studies performed to date, it would appear that a high proportion of $R B 1$ mutations with reduced penetrance are splice site mutations, although not all splice site mutations display low penetrance.

Some mutations are associated with specifically reduced penetrance as compared to other mutations of the same type in the same gene. For example, BRCAl Arg1699Gln is characterized by a cumulative risk of breast or ovarian cancer by the age of 70 years of only $24 \%$ (Spurdle et al. 2012), much lower than for the average pathogenic BRCA1 mutation ( $\sim 71 \%$; van der Kolk et al. 2010). Individuals with GJB2-associated deafness who harbour two nonsense/ truncating mutations exhibit a much more severe clinical phenotype, and hence are more likely to come to clinical attention, than those harbouring two missense mutations (Azaiez et al. 2004). This serves to illustrate that, in recessive disorders, the clinical penetrance of one mutation may be strongly influenced by the nature of the other mutation in trans. In Ehlers-Danlos syndrome type IV, however, null COL3A1 mutations tend to exhibit lower penetrance than missense and splicing mutations (Leistritz et al. 2011); this is presumably because a faulty gene product can disrupt the entire triple helical collagen molecule, whereas a null mutation merely reduces the amount of normal collagen produced (Arnold and Fertala 2013). In the same vein, patients with heritable pulmonary arterial hypertension, due to missense mutations in the $B M P R 2$ gene, present earlier and with more severe disease than patients harbouring truncating mutations (Austin et al. 2009a). It is thought that the missense mutations are associated with stable BMPR2 transcripts encoding BMPR2 protein which exerts a dominant negative effect on BMP signalling, thereby rendering missense mutations more detrimental than truncating mutations. The majority of $B M P R 2$ missense mutations were penetrant prior to the age of 36 years, whereas the majority of truncating mutations became penetrant only after the age of 36 years
(Austin et al. 2009a). With mutations of the TNFRSF1A gene causing TNF receptor-associated periodic syndrome, missense mutations in cysteine residues have been reported to be more penetrant than missense mutations in non-cysteine residues (Aksentijevich et al. 2001; Aganna et al. 2001). Intriguingly, a SOD1 Leu117Val missense mutation which yields a mutant protein indistinguishable from wildtype SOD1 (in terms of its activity, stability and folding) causes amyotrophic lateral sclerosis, but with unusually low penetrance and slow progression (Synofzik et al. 2012). Finally, clinical penetrance may vary not only with the mutation type, but also with the location of the mutation in the gene/protein (Jackson et al. 1999; Risch et al. 2001; Yatsenko et al. 2001; van der Werf et al. 2012; Ho et al. 2012).

In autosomal dominant hereditary pancreatitis, the penetrance of the PRSSI Arg122His mutation has been calculated to be $86 \%$, whereas that of PRSS1 Ala16Val is of the order of 55-65 \% (Grocock et al. 2010; Joergensen et al. 2010). It is thought that the comparatively low penetrance of this latter mutation may be related to its particularly mild biochemical phenotype. The Ala16Val substitution alters the $N$-terminal residue of the trypsinogen activation peptide, thereby increasing the rate of $N$-terminal processing by chymotrypsin C by $\sim 5.8$-fold (Szabó and Sahin-Tóth 2012). Since the activation peptide is released during the activation process, the Ala16Val mutation is absent from active trypsin and hence cannot influence trypsin function.

Missense mutations in the $M E F V$ gene responsible for familial Mediterranean fever can differ quite dramatically in terms of their clinical penetrance. For example, Met694Val is generally characterized by high penetrance, whereas both Glu148GIn and Val726Ala exhibit reduced penetrance (Shohat and Halpern 2011). In this disorder, the carrier frequency is higher than would be expected from the prevalence of the disease, suggesting that the penetrance of pathogenic $M E F V$ mutations may often be incomplete in the compound heterozygous state (GershoniBaruch et al. 2002; Zaks et al. 2003; Caglayan et al. 2010; Camus et al. 2012; Soriano and Manna 2012).

Double missense mutations in cis are not infrequently encountered in patients with an inherited disease. One of the two mutations may represent a hypomorphic (i.e. less functional) allele, as for example with the GLA Asp313Tyr occurring in cis to the pathogenic Gly411Asp in patients with Fabry disease (Yasuda et al. 2003). Double missense mutations in cis may however be associated with a highly variable clinical phenotype (e.g. MEFV, Pro369Ser/ Arg408Gln as a cause of familial Mediterranean fever; Ryan et al. 2010). A low-penetrance missense mutation may be associated with a particularly severe clinical phenotype when it occurs in cis with a second known pathogenic mutation, e.g. MYH7 Val606Met and Ala728Val in hypertrophic cardiomyopathy (Blair et al. 2001). Similarly, 
two missense mutations in cis, each individually exerting a comparatively mild or no effect on the clinical phenotype, can act in concert leading to a more severe effect on the phenotype than either acting alone (e.g. CFTR Arg347His and Asp979Ala in cystic fibrosis; Clain et al. 2001 or RET Cys634Tyr and Tyr791Phe resulting in pheochromocytoma with high penetrance; Toledo et al. 2010). By contrast, Brugnoni et al. (2013) have intriguingly claimed that two different $C L C N 1$ mutations do not give rise to myotonia congenita when they occur in cis on the same allele, although both lesions cause the disease when inherited on their own.

In diseases that exhibit locus heterogeneity, clinical penetrance may vary between mutations in different genes. For example, in pancreatitis, penetrance may vary from virtually $100 \%$ in the case of the most common mutations in the cationic trypsinogen gene (PRSS1) gene, via an intermediate level for SPINKI and CFTR mutations, to the much more subtle risk conferred by the disease modifiers, namely variants in the chymotrypsin $\mathrm{C}(C T R C)$, calciumsensing receptor (CASR) and anionic trypsin (PRSS2) genes, which can only be identified through large cohort studies (Lerch et al. 2010). It should, however, be noted that in cases where mutations in the SPINKI and CASR genes (Felderbauer et al. 2003) or SPINK1 and CFTR genes (Masson et al. 2007) are co-inherited, chronic pancreatitis can ensue. Other such examples of digenic inheritance are discussed below (see digenic mutations and disease penetrance).

Reduced penetrance alleles are also characteristic of many triplet repeat expansion disorders. For example, in Huntington disease, the possession of intragenic (HTT) CAG repeats of 36-39 copies (in $0.01 \%$ of controls and $\sim 5 \%$ of consultands) is often associated with reduced penetrance manifesting as a later onset of clinical symptoms (McNeil et al. 1997; Quarrell et al. 2007; Sequeiros et al. 2010; Panegyres and Goh 2011; Huntington Study Group COHORT Investigators 2012). Contractions of the expanded CAG repeat length below a certain threshold can occasionally be responsible even for the non-occurrence of Huntington disease in a given at-risk individual (Nahhas et al. 2009). However, it should be appreciated that a substantial proportion of the variance in age of onset in Huntington disease is due either to variation in genes other than $H T T$ or in the environment (Wexler et al. 2004). Other repeat expansion disorders characterized by reduced penetrance of alleles of intermediate size include fragile $\mathrm{X}$-associated tremor/ataxia syndrome (Jacquemont et al. 2004; Sévin et al. 2009), spinocerebellar ataxia types 10 (Alonso et al. 2006; Rankin et al. 2008) and 17 (Oda et al. 2004; Nolte et al. 2010), inherited prion disease (Kaski et al. 2011) and amyotrophic lateral sclerosis (Boeve et al. 2012; Ogaki et al. 2012).

\section{Modulating influence of additional allelic variants in cis or in trans}

Some allelic variants may influence the expression of their host gene so as to alter the penetrance of a potentially pathological mutation in the same gene. Such modulatory variants may reside within exons, introns or regulatory regions. For example, the common Arg413Gln F7 polymorphism (Arg353Gln in legacy nomenclature; rs6046), which serves to reduce the level of secreted coagulation factor VII by $\sim 25 \%$ (Arbini et al. 1994; Hunault et al. 1997), is over-represented among individuals with clinically symptomatic factor VII deficiency (Millar et al. 2000). This is consistent with the view that Arg413Gln is a functional polymorphism and that the presence of Gln 413 increases the likelihood that an individual, whose haemostatic potential is already compromised by a heterozygous F7 mutation, will come to clinical attention due to a bleeding diathesis. The same principle applies to the functional Arg202Gln MEFV polymorphism (rs224222) where the Gln202 allele occurs in the homozygous state at a disproportionately higher frequency $(15 \%)$ in familial Mediterranean fever patients than in normal controls (2.7\%) (Yigit et al. 2012). In similar vein, the $\mathrm{T}$ allele of a functional $\mathrm{C} / \mathrm{T}$ polymorphism (rs11024595) in the promoter region of the SAAl gene is significantly over-represented in familial Mediterranean fever patients as compared with normal controls (Migita et al. 2013). The MLH1 Lys618Ala mutation (AAG $>$ GCG; rs35502531), initially supposed to be a benign polymorphism, has been found to be significantly over-represented in sporadic cancers associated with Lynch syndrome; MLH1 Ala618 appears to have a reduced ability to bind PMS2, one of the MLH1 protein's mismatch repair partners (Medeiros et al. 2012). Finally, the functional KCNE1 Asp85Asn polymorphism (rs1805128), which occurs in the general population with a frequency of $0.8 \%$, occurs at a frequency of $3.9 \%$ in long QT syndrome patients (Nishio et al. 2009).

Various reported examples of the modulation of the impact of pathogenic missense mutations by allelic single nucleotide polymorphisms (SNPs) are given in Table 2. Thus, the missense polymorphism Asp216His (rs1801968) in the TOR1A (DYT1) gene serves to moderate the clinical impact, in both cis and in trans, of the TORIA c.904-906 del GAG mutation, the major mutation underlying earlyonset dystonia (Kock et al. 2006; Risch et al. 2007; Martino et al. 2013). Similarly, in long QT syndrome, the genotype of a missense polymorphism (Lys897Thr; rs1805123) in the $\mathrm{KCNH} 2$ gene appears to distinguish symptomatic from asymptomatic individuals carrying a low-penetrance Ala1116Val pathogenic mutation (Crotti et al. 2005). Another example of the modulatory effect of a missense polymorphism on disease allele penetrance is provided by 
Table 2 Examples of pathogenic microlesions whose penetrance has been found to be modulated by allelic SNPs

\begin{tabular}{|c|c|c|c|c|}
\hline Disease & Gene & Pathological mutation & Modifying SNP & Reference \\
\hline Factor VII deficiency & $F 7$ & Various & $\begin{array}{l}\text { NM_000131.3: c.1238G >A Arg413Gln } \\
\text { (rs6046) }\end{array}$ & Millar et al. (2000) \\
\hline Brugada syndrome & $S C N 5 A$ & NM_198056.2: c.5243G>A Gly1748Asp & $\begin{array}{l}\text { NM_198056.2: c.1673A }>\text { G His558Arg } \\
\quad(\text { rs1805124) }\end{array}$ & Núñez et al. (2013) \\
\hline Familial sick sinus syndrome & $S C N 5 A$ & Various & $\begin{array}{l}\text { NM_198056.2: c.1673A }>\text { G His558Arg } \\
\quad(r s 1805124)\end{array}$ & Gui et al. (2010) \\
\hline Early-onset dystonia & TORIA & NM_000113.2: c.907-909delGAG & $\begin{array}{l}\text { NM_000113.2: c.646G }>\text { C Asp216His } \\
\quad(\text { rs1801968) }\end{array}$ & $\begin{array}{l}\text { Kock et al. (2006) and } \\
\text { Risch et al. (2007) }\end{array}$ \\
\hline Familial Mediterranean fever & $M E F V$ & Various & $\begin{array}{l}\text { NM_000243.2: c.605G >A Arg202Gln } \\
\text { (rs224222) }\end{array}$ & Yigit et al. (2012) \\
\hline Lynch syndrome & $M L H 1$ & Various & $\begin{array}{l}\text { NM_000249.3: } \\
\text { c.1852_1853delAAinsGC Lys618Ala } \\
\text { (rs35502531) }\end{array}$ & Medeiros et al. (2012) \\
\hline Long QT syndrome & $\mathrm{KCNH} 2$ & NM_000238.3: c.3347C>T Ala1116Val & $\begin{array}{l}\text { NM_000238.3: c.2690A >C Lys897Thr } \\
\quad(\text { rs1805123) }\end{array}$ & Crotti et al. (2005) \\
\hline Long QT syndrome & $\mathrm{KCNH} 2$ & NM_000238.3: c.1468G >A Ala490Thr & $\begin{array}{l}\text { NM_000238.3: c.2690A >C Lys897Thr } \\
\quad(\text { rs1805123) }\end{array}$ & Zhang et al. (2008) \\
\hline $\begin{array}{l}\text { Creutzfeld-Jakob disease/ } \\
\text { fatal familial insomnia }\end{array}$ & $P R N P$ & NM_000311.3: c.532G>A Asp178Asn & $\begin{array}{l}\text { NM_000311.3: c.385A>G Met129Val } \\
\text { (rs1799990) }\end{array}$ & $\begin{array}{l}\text { Goldfarb et al. (1992) and } \\
\text { Apetri et al. (2005) }\end{array}$ \\
\hline $\begin{array}{l}\text { Cardiac conduction } \\
\text { abnormalities/sudden death }\end{array}$ & $S C N 5 A$ & $\begin{array}{l}\text { NM_198056.2: c.4262G }>\text { A } \\
\text { Trp1421Term }\end{array}$ & $\begin{array}{l}\text { NM_198056.2: c.3578G >A Arg1193Gln } \\
\quad(\text { rs41261344) }\end{array}$ & Niu et al. (2006) \\
\hline Sudden unexplained death & $S C N 5 A$ & NM_198056.2: c.2039G >A Arg680His & $\begin{array}{l}\text { NM_198056.2: c.3308C>A Ser1103Tyr } \\
\text { (rs7626962) }\end{array}$ & Cheng et al. (2011) \\
\hline Syncope & $S C N 5 A$ & NM_198056.2: c.5851G >T Val1951Leu & $\begin{array}{l}\text { NM_198056.2: c.1673A }>\text { G His558Arg } \\
\quad(\text { rs1805124) }\end{array}$ & $\begin{array}{l}\text { Shinlapawittayatorn et al. } \\
\text { (2011) }\end{array}$ \\
\hline Medullary thyroid carcinoma & $R E T$ & NM_020975.4: c.1996A>G Lys666Glu & $\begin{array}{l}\text { NM_020975.4: c.2071G>A Gly691Ser } \\
\text { (rs1799939) }\end{array}$ & Borrello et al. (2011) \\
\hline Medullary thyroid carcinoma & $R E T$ & NM_020975.4: c.1597G>T Gly533Cys & $\begin{array}{l}\text { NM_020975.4: c.74-126G }>\mathrm{T} \\
\quad(\text { rs } 2565206)\end{array}$ & Tamanaha et al. (2009) \\
\hline Homocystinuria & $M T R R$ & NM_002454.2: c.166G >A Val56Met & $\begin{array}{l}\text { NM_002454.2: c.66G }>\text { A Ile22Met } \\
\text { (rs1801394) }\end{array}$ & Gherasim et al. (2007) \\
\hline Childhood absence epilepsy & CACNAIH & NM_021098.2: c.2318G >A Gly773Asp & $\begin{array}{l}\text { NM_021098.2: c.2362C }>\text { T Arg788Cys } \\
\quad(\mathrm{rs} 3751664)\end{array}$ & Vitko et al. (2005) \\
\hline Primary hyperoxaluria type 1 & $A G X T$ & NM_000030.2: c.731T>C Ile244Thr & $\begin{array}{l}\text { NM_000030.2: c.32C }>\text { T Pro11Leu } \\
\quad(\mathrm{rs} 34116584)\end{array}$ & Santana et al. (2003) \\
\hline Hereditary spherocytosis & SPTA1 & $\begin{array}{l}\text { NM_003126.2: c.7134G }>\text { A Gln2377Gln, } \\
\text { alters splicing of exon } 51\end{array}$ & c. $4339-99 \mathrm{C}>\mathrm{T}(\mathrm{rs} 200830867)$ & Delaunay et al. (2004) \\
\hline $\begin{array}{l}\text { Erythropoietic } \\
\text { protoporphyria }\end{array}$ & FECH & Various & $\begin{array}{l}\text { NM_000140.3: c.315-48T }>C \\
\quad(\text { rs2272783) }\end{array}$ & Gouya et al. (2006) \\
\hline $\begin{array}{l}\text { Autosomal dominant } \\
\text { osteopetrosis type II }\end{array}$ & CLCN7 & Various & $\begin{array}{l}\text { NM_001287.4: c.1252G }>\text { A Val418Met } \\
\quad(\text { rs12926089) }\end{array}$ & Chu et al. (2005) \\
\hline $\begin{array}{l}\text { Haemolytic uraemic } \\
\text { syndrome }\end{array}$ & $\mathrm{CFH}$ & Various & $\begin{array}{l}\text { NM_000186.3: c.2808G > T Glu936Asp } \\
\quad(\text { rs1065489) }\end{array}$ & Caprioli et al. (2003) \\
\hline Hereditary spastic paraplegia & SPAST & $\begin{array}{l}\text { NM_014946.3: c.1687G }>\text { A, (alters } \\
\text { splicing of exon 15) }\end{array}$ & $\begin{array}{l}\text { NM_014946.3: c.131C >T Ser44Leu } \\
\quad(\mathrm{rs} 121908515)\end{array}$ & Pantakani et al. (2008) \\
\hline GM1 gangliosidosis & $G L B 1$ & NM_000404.2: c.601C>T Arg201Cys & $\begin{array}{l}\text { NM_000404.2: c.1306C }>\text { T Leu436Phe } \\
\quad(\text { rs34421970) }\end{array}$ & Caciotti et al. (2003) \\
\hline Primary cortisol resistance & $N R 3 C 1$ & $\begin{array}{l}\text { NM_001018077.1: c.2035G >A } \\
\text { Gly679Ser }\end{array}$ & $\begin{array}{l}\text { NM_001018077.1: c.68G >A Arg23Lys } \\
\text { (rs6190) }\end{array}$ & Raef et al. (2008) \\
\hline Atopic dermatitis & SPINK5 & NM_006846.3: c.2468dupA & $\begin{array}{l}\text { NM_006846.3: c.1258G >A Glu420Lys } \\
\quad(\text { rs2303067) }\end{array}$ & Di et al. (2009) \\
\hline Hyperinsulinism & $H A D H$ & NM_005327.4: c.636+471G >T & $\begin{array}{l}\text { NM_005327.4: c.636+385A }>G \\
\quad(\text { rs732941) }\end{array}$ & Flanagan et al. (2013) \\
\hline
\end{tabular}

the His558Arg substitution (rs1805124) in the SCN5A gene in a case of Brugada syndrome type 1 caused by compound heterozygous mutations (Asp1690Asn and Gly1748Asp) in
SCN5A. Both mutations reduced the peak $\mathrm{Na}^{+}$current density due to limited trafficking of the SCN5A protein towards the membrane, but Gly1748Asp also profoundly 
affected channel gating. The His558Arg polymorphism was found to be capable of rescuing the defective trafficking of SCN5A Asn1690 towards the membrane when present in cis to the pathological lesion (Núñez et al. 2013). Intriguingly, cotransfection with Asn1690, either alone or together with the modulatory His558Arg polymorphism, completely restored the gating defect associated with the pathogenic Gly1748Asp mutation in trans, although it only slightly rescued its trafficking.

In passing, it is perhaps pertinent to note that interplay between functional SNPs may, depending upon the precise combination of alleles involved, also be sufficient to bring about disease in the absence of a pathogenic mutation sensu stricto. Thus, a particular allele of one SNP may contribute to pathogenesis, but only in the presence of specific allele of another SNP. These SNPs may be neighbouring, as in the case of Glu918Asp (rs16022) and Glu993Val (rs16023) in the CACNA1A gene, which appear to contribute to migraine susceptibility (D'Onofrio et al. 2009). In the same way, when they occur together in cis, two otherwise neutral missense polymorphisms in the FMO3 gene [Glu158Lys (rs2266782) and Glu308Gly (rs2266780)] result in a decrease in FMO3 enzymatic activity that is sufficient to give rise to a mild form of trimethylaminuria (Akerman et al. 1999; Zschocke et al. 1999; D'Angelo et al. 2013). However, the interacting SNPs can also be located within different genes as in the case of Pro589Ser (rs1049296) in the transferrin $(T F)$ gene and Cys282Tyr (rs1800562) in the haemochromatosis (HFE) gene which interact so as to increase the risk of Alzheimer disease (Robson et al. 2004; Kauwe et al. 2010). A further example of the combined effect of two unlinked SNPs is provided by an intronic SNP in the thrombospondin 2 (THBS2) gene (c.1478$8 \mathrm{C}>\mathrm{T}$; rs 9406328) and a missense SNP in the metalloproteinase 9 (MMP9) gene (Gln279Arg; rs17576), which together increase the risk of lumbar disc herniation (Hirose et al. 2008). This type of situation may occupy the middle ground between monogenic and complex disorders.

The modifying SNP may also be regulatory in nature and can serve to render the pathogenic coding mutation more or less deleterious (and hence more or less penetrant) depending upon whether the allele harbouring it is more or less expressed than the wild-type allele. An example of this is provided by the $-30 \mathrm{C}>\mathrm{T}$ variant ( $\mathrm{rs} 17249141)$ in the $L D L R$ gene promoter that has been shown to act in concert with a low-penetrant missense mutation in cis so as to give rise to an unusually severe form of familial hypercholesterolaemia (Snozek et al. 2009). The modulating influence of regulatory SNPs on the penetrance of coding mutations located in cis appears to be a widespread phenomenon in medical genetics, with the high expressing SNP allele usually increasing the clinical penetrance of the linked coding mutation (Lappalainen et al. 2011). One regulatory SNP is thought to act as a low-penetrance cancer susceptibility factor in its own right: homozygosity for the intronic 309T $>$ G MDM2 variant (rs2279744), which leads to enhanced binding of the Sp1 transcription factor and $M D M 2$ up-regulation, appears to increase the risk for many types of tumour, presumably in concert with other lesions (Hu et al. 2007).

Introns may also harbour SNPs that are capable of modulating the clinical penetrance of a given pathogenic mutation. Thus, a short tract of five thymidines (5T) in intron 8 of the CFTR gene, found in $\sim 10 \%$ of individuals from the general population, can give rise to either congenital absence of the vas deferens (CAVD), non-classical cystic fibrosis or a normal phenotype when found in trans to a severe CFTR mutation (Kiesewetter et al. 1993; Cuppens et al. 1998). The number of TG repeats immediately adjacent to $5 \mathrm{~T}$ is not only significantly associated with the level of alternative splicing of exon 9 of the CFTR gene (Cuppens et al. 1998; Niksic et al. 1999), but also influences clinical penetrance both in the context of cystic fibrosis and CAVD (Groman et al. 2004; Buratti et al. 2004; Lebo and Grody 2007). Another intronic modifying polymorphism is found in the $F E C H$ gene responsible for erythropoietic protoporphyria, an autosomal dominant disorder characterized by incomplete penetrance. This polymorphism (c.315-48T $>$ C; rs2272783) modulates the use of a cryptic acceptor splice site, yielding an aberrantly spliced FECH mRNA which is degraded via nonsensemediated mRNA decay (Gouya et al. 1999, 2002). The hypomorphic $\mathrm{C}$ allele increases the penetrance of erythropoietic protoporphyria when it occurs in trans to a pathogenic FECH mutation (Gouya et al. 2002, 2006). Finally, in a family with hyperinsulinism, a c.636+385A $>$ G SNP (rs732941) in intron 5 of the $H A D H$ gene, which creates a cryptic acceptor splice site, acts in concert with a pathogenic $H A D H$ mutation $(\mathrm{c} .636+471 \mathrm{G}>\mathrm{T})$ in the same intron, which creates a cryptic donor splice site, to generate a 141-bp pseudoexon that leads to premature termination of translation (Flanagan et al. 2013).

A common $\mathrm{C}>\mathrm{T}$ variant within an enhancer in intron 1 of the RET gene (rs2435357) serves to increase the clinical penetrance of $R E T$ coding sequence mutations (Emison et al. 2005). The T allele disrupts a SOX10-binding site, thereby reducing RET transactivation (Emison et al. 2010). A comparable example is provided by an intronic enhancer SNP (rs2596623) in the thyroid hormone receptor $\beta$ (THRB) gene, which was found to be responsible for the pituitary cell-specific over-expression of a mutant thyroid hormone receptor $\beta 2$ (Arg338Trp) in a case of pituitary cell-specific resistance to thyroid hormone (Alberobello et al. 2011).

Variants in the $3^{\prime}$ untranslated region $\left(3^{\prime} \mathrm{UTR}\right)$ of the $K C N Q 1$ gene reportedly modify disease severity in individuals with type 1 long QT syndrome resulting from KCNQ1 gene mutations (Amin et al. 2012). These variants 
serve to reduce $K C N Q 1$ gene expression, such that patients with one or more variants on their mutated $K C N Q 1$ alleles have a shorter QT interval and a milder clinical phenotype, whereas patients with the variants on their normal $K C N Q 1$ alleles exhibit significantly longer QT intervals and a more severe clinical phenotype. Another example of a modifying $3^{\prime}$ UTR variant is provided by the G $>$ A polymorphism (rs1799963) at position 20210 in the prothrombin (F2) gene, which increases the risk of venous thrombosis by enhancing $F 2$ mRNA $3^{\prime}$ end formation efficiency, thereby boosting thrombin formation (Gehring et al. 2001). This $F 2$ $20210 \mathrm{G}>\mathrm{A}$ polymorphism has been claimed to interact with a common F13Al Val34Leu (rs5985) variant to confer a greatly increased risk of myocardial infarction (Butt et al. 2003). Some 3'UTR variants are located within microRNA-binding sites and may constitute low-penetrance risk factors for disease in their own right (Ahluwalia et al. 2009; Kontorovich et al. 2010; Qiu et al. 2011; Arnold et al. 2012). Finally, various SNPs in other noncoding RNAs (e.g. lincRNAs; Jendrzejewski et al. 2012; Kumar et al. 2013) appear to be disease associated and may therefore also influence disease penetrance.

\section{Influence of the gene expression level on mutation penetrance}

Humans are characterized by marked inter-individual differences in the expression levels of their genes (Stranger et al. 2007; Skelly et al. 2009; Cowley et al. 2009; Cheng et al. 2012). Since gene expression is controlled by a combination of cis- and trans-acting regulatory factors, one means by which heritable differences in gene expression may be mediated is through polymorphism either of transacting regulatory (transcription) factors or of the cis-acting target sequences to which they bind. In the case of disease genes, such inter-individual variation in gene expression levels and patterns can influence the penetrance of pathological mutations. However, it should be appreciated that there are also substantial environmental and stochastic (non-genetic) components to gene expression that are likely to contribute to variable penetrance, even between monozygotic twins (Grundberg et al. 2012).

Differential allelic expression is a widespread phenomenon and is thought to be relevant to as many as $50 \%$ of all human genes (Williams et al. 2007; Cheung and Spielman 2009; Palacios et al. 2009). In autosomal dominant conditions where the two alleles of the disease gene are expressed at different levels, this discrepancy can favour either the mutant or the wild-type allele and hence may influence clinical penetrance in either direction (de la Chapelle 2009). Thus, in pulmonary arterial hypertension, a disease caused by mutations in the bone morphogenetic protein receptor type 2 (BMPR2) gene, the penetrance of the $B M P R 2$ disease allele is dependent upon the level of expression of the wildtype BMPR2 allele (Hamid et al. 2009a). Similarly, in erythropoietic protoporphyria, an autosomal dominant condition caused by mutations in the ferrochelatase $(F E C H)$ gene, the penetrance of the pathogenic FECH allele is influenced by the level of expression of the wild-type $F E C H$ allele (Gouya et al. 1999; 2002; Di Pierro et al. 2007). Other examples of autosomal dominant conditions where the degree of clinical penetrance is modulated by differential expression of the wild-type and mutant alleles include hereditary elliptocytosis (SPTA1, Wilmotte et al. 1993), Marfan syndrome (FBNI, Hutchinson et al. 2003), retinoblastoma (RB1, Taylor et al. 2007), colorectal cancer (APC, Yan et al. 2002; TGFBRl, Valle et al. 2008) and breast and ovarian cancer (BRCA1, Ginolhac et al. 2003).

Perhaps, the best understood example of penetrance depending upon the level of expression of the wild-type allele is retinitis pigmentosa type 11 (Utz et al. 2013). This autosomal dominant condition is caused by mutations in the pre-mRNA processing factor 31 (PRPF31) gene located on chromosome 19q13.42. The clinical penetrance of the underlying mutations has been shown to depend upon the level of wild-type PRPF31 mRNA expression displayed by the patient (Vithana et al. 2003; Rivolta et al. 2006; Liu et al. 2008). Cells from asymptomatic carriers of PRPF31 mutations express a higher level of the wild-type allele than cells from affected patients: high enough for the wild-type PRPF31 mRNA level to lie within the range of the unaffected general population (Rivolta et al. 2006; Liu et al. 2008). The penetrance of PRPF31 mutations is reduced by transcriptional repression mediated by the product of the CCR4-NOT transcription complex, subunit 3 (CNOT3) gene which is linked to PRPF31 (McGee et al. 1997; Venturini et al. 2012). PRPF31 expression has also been found to be strongly influenced by an unlinked eQTL on chromosome 14q21-q23 (Rio Frio et al. 2008). The penetrance of PRPF31 mutations is therefore determined at least in part by a trans-acting modifier located on a different chromosome. The trans-acting alleles are inherited from the parent lacking the PRPF31 mutation; these alleles are presumably present in the general population, but appear only to be relevant to disease when they modulate the penetrance of PRPF31 mutations.

A slightly different scenario is exemplified by Schimke immune-osseus dysplasia (SIOD), a recessive condition, which appears to result from biallelic mutations in the SMARCAL1 gene. Several examples of SIOD families with incomplete penetrance have been reported (Bökenkamp et al. 2005; Dekel et al. 2008; Elizondo et al. 2009). It has recently been shown that SMARCAL1, a protein involved in chromatin remodelling, influences the transcription level of many genes (Baradaran-Heravi et al. 2012). Although 
SMARCAL1 deficiency is insufficient in itself to cause SIOD in Drosophila and mouse models, the addition of environmental (viz. heat shock) and genetic insults affecting transcription can successfully recapitulate the pathophysiology of SIOD (Baradaran-Heravi et al. 2012). The penetrance of SIOD therefore appears to be dependent upon the magnitude of the alteration of gene expression consequent to SMARCAL1 deficiency.

In the case of a splicing mutation (c.291+1 G>A; rs71640277) in intron 3 of the $G H 1$ gene causing growth hormone deficiency type II, the expression levels of the mutant and wild-type alleles were found to correlate with the penetrance and expressivity of the deficiency state in different members of the same family (Hamid et al. 2009b). Some splicing mutations associated with low penetrance affect splicing in such a way that both normal-length and truncated transcripts are expressed from the same mutant allele, but presumably to different extents in different individuals (e.g. $R B 1$, c.2211G $>$ A; Schubert et al. 1997). Other such examples of reduced penetrance due to 'leaky splicing' involve splicing mutations in the SPAST gene causing hereditary spastic paraplegia (Svenson et al. 2001) and in the $B T K$ gene causing $\mathrm{X}$-linked agammaglobulinaemia (Kaneko et al. 2005). The reduced penetrance characteristic of some splicing mutations may also result from alternative splicing (Rave-Harel et al. 1997; Chiba-Falek et al. 1998; Nissim-Rafinia and Kerem 2005; Zinman et al. 2009; Szymanski et al. 2011; Cogan et al. 2012; Lee et al. 2012b) or internal translational start site initiation (Sánchez-Sánchez et al. 2007).

Special cases of differential allelic expression are of course provided by X-inactivation (Dobyns et al. 2004) and imprinting (Lo et al. 2003), both of which are discussed below.

\section{Allele dosage and its influence on penetrance}

Formally, use of the term 'autosomal dominant' implies that the homozygotes exhibit the same or a similar clinical phenotype to the heterozygotes, as is the case in Huntington disease where the length of the expanded HTT CAG triplet repeat appears to be predictive of the age of onset irrespective of the presence or absence of a second expanded HTT allele (Lee et al. 2012a). However, in practice, for most 'dominant' human disorders in which homozygotes have been reported, their clinical symptoms tend to be significantly more severe than in the heterozygotes (Vogel and Motulsky 1997). This would seem to be especially true in the context of low-penetrance mutations such as those identified in the SCN4A and CLCN1 genes, causing muscle channelopathies, conditions which are usually held to be transmitted in an autosomal dominant fashion. Patients homozygous for sodium channel mutations causing paramyotonia congenita (SCN4A, Ile1393Thr), hypokalemic periodic paralysis (SCN4A, Arg1132Gln) and myotonia congenita (CLCN1, Gly190Ser, Ile556Asn, Ala313Thr, Ile556Asn) display much more severe clinical features than patients heterozygous for these mutations (Plassart-Schiess et al. 1998; Arzel-Hézode et al. 2010; Shalata et al. 2010). The aforementioned mutations were also found to exhibit reduced penetrance in heterozygotes.

Mutations in the RET gene, associated with isolated Hirschsprung disease, are dominant loss-of-function mutations with incomplete penetrance and variable expressivity. BaselVanagaite et al. (2007) reported a c.1263+5G $>$ A splicing mutation in the homozygous state in three females with severe Hirschsprung disease and in the heterozygous state in a male patient with short-segment Hirschsprung disease. In addition, a hypomorphic RET-predisposing allele, rs2435357, located in the first intron of the RET gene, was found in the heterozygous state in the male patient but not in the three affected females. Whilst the heterozygous c. $1263+5 \mathrm{G}>\mathrm{A}$ mutation is known to be low penetrance for short-segment Hirschsprung disease, the homozygous state is fully penetrant for total aganglionosis or long-segment Hirschsprung disease. Thus, the penetrance of RET gene mutations in Hirschsprung disease depends not only on the nature of the mutation but also on the allele dosage.

\section{Influence of copy number variation on mutation penetrance}

Estimates of the clinical penetrance of recurrent pathogenic copy number variants (CNVs) vary quite widely, depending upon CNV size, genomic location and the disorder in question (Ben-Shachar et al. 2009; Vassos et al. 2010; Breckpot et al. 2011; Čiuladaitè et al. 2011; Hosak et al. 2012; Klopocki et al. 2012; Rosenfeld et al. 2013; Vaags et al. 2012; Weischenfeldt et al. 2013; Dabell et al. 2013; Carvill and Mefford 2013; Tropeano et al. 2013). In their study of children known to carry a CNV associated with intellectual disability and congenital abnormalities, Girirajan et al. (2012) reported synergy between multiple large CNVs leading to a particularly severe clinical presentation. Such a two-hit model, or 'oligogenic heterozygosity' as it has been termed, also appears to be characteristic of autism (Pinto et al. 2010; Schaaf et al. 2011b; Klei et al. 2012; Gau et al. 2012).

The penetrance of a given CNV may also be influenced by genetic variants in the vicinity. Thus, a submicroscopic deletion of $1 \mathrm{q} 21.1$ (encompassing the RBM $8 A$ gene) has been reported to interact with a low-frequency functional $\mathrm{SNP}$ in the regulatory region of the wild-type $R B M 8 A$ allele to cause thrombocytopaenia with absent radii (Albers et al. 2012). 
Several papers have now suggested that CNVs can also act as genetic modifiers of phenotype severity in a variety of different disease contexts (Beckmann et al. 2007; Chaudru et al. 2009; El-Hattab et al. 2010; Mulley et al. 2011; Jiang et al. 2011; Carvalho et al. 2012; Shen et al. 2013b). CNVs may influence the penetrance of a clinical phenotype indirectly as well as directly. For example, a mutant gene might be 'covered' by a CNV in a given individual, so that the expected clinical phenotype would be masked by the presence of an additional wild-type copy in cis to the gene in question. Consistent with this postulate, $\mathrm{Ng}$ et al. (2008) reported that $\sim 30 \%$ of nonsense SNPs occur in genes residing within segmental duplications, a proportion some threefold larger than that noted for synonymous SNPs. Genes harbouring nonsense SNPs were also found to belong to larger gene families ( $\mathrm{Ng}$ et al. 2008) suggesting that some functional redundancy could also exist between paralogous human genes. In support of this idea, Hsiao and Vitkup (2008) reported that those human genes which have a homologue with $>90 \%$ sequence similarity are $\sim 3$ times less likely to harbour disease-causing mutations than genes with less closely related homologues. Hsiao and Vitkup (2008) interpreted their findings in terms of 'genetic robustness' against null mutations, with the duplicated sequences providing 'backup' by potentiating the functional compensation/complementation of homologous genes in the event that the latter acquired deleterious mutations. The capacity to be functionally compensated appears to vary, in the order non-disease genes $>$ monogenic disease genes $>$ polygenic disease genes (Podder and Ghosh 2011). One example of how a CNV can ameliorate the clinical phenotype is spinal muscular atrophy where an increased copy number of the SMN2 gene can greatly reduce the severity of the disease caused by the homozygous deletion of the SMN1 gene, because the SMN2 gene, which lacks a splicing enhancer, can nevertheless generate some functional product thereby compensating functionally in a copy numberdependent fashion for the loss of the SMN1 gene (Vitali et al. 1999; Harada et al. 2002; Wirth et al. 2006). Another example of how the clinical and/or phenotypic impact of a mutant gene can be nullified by a $\mathrm{CNV}$ is provided by a foetus that possessed paternal (Gln318Term) and maternal (Arg356Term) nonsense mutations of the CYP21A2 gene but lacked the normal clinical sequelae of congenital adrenal hyperplasia; this was found to be due to a duplication of the CYP21A2 gene on the paternal allele (Kleinle et al. 2009; Lekarev et al. 2013).

\section{Influence of modifier genes on disease penetrance}

"For a so-called single gene disorder, there is one gene that may be primarily responsible for the pathogenesis with one or more independently inherited modifier genes that influence the phenotype. On the other hand, for a complex trait, the primacy of any individual gene is not perceptible, and the interaction of two or more independently inherited pairs of alleles, most likely influenced by additional modifier genes, results in the disease. The consequence of this conceptual framework is that there is no such thing as a 'single' gene disorder. In other words, there is no obvious clear distinction between simple Mendelian and complex traits: genetic diseases represent a continuum with diminishing influence from a single primary gene influenced by modifier genes, to increasingly shared influence by multiple genes".

Dipple and McCabe (2000)

It is sometimes claimed that sickle cell anaemia is the simplest of all Mendelian disorders in that it is caused by one specific mutation (Glu7Val) in the $\beta$-globin $(H B B)$ gene. However, this single-mutation monogenic disorder is not as simple as it might at first appear; indeed, it is characterized by marked clinical heterogeneity and incomplete penetrance of subphenotypes which is due in part to allelic variation and in part to variants in unlinked modifier genes (Steinberg and Sebastiani 2012). If we extrapolate from the archetypal example of sickle cell disease to other Mendelian disorders, it is not unreasonable to expect the action of modifier genes to be the rule rather than the exception. Indeed, variants in unlinked modifier genes have been reported to influence penetrance in a variety of different inherited diseases including pancreatitis (Khalid et al. 2006), breast cancer (Wolf et al. 2010; Wang et al. 2010a; Walker et al. 2010; Antoniou and ChenevixTrench 2010; Esteban Cardeñosa et al. 2012; Harlid et al. 2012), Gaucher disease (Taddei et al. 2009; Zhang et al. 2012), retinitis pigmentosa (Rio Frio et al. 2008; Venturini et al. 2012), haemochromatosis (Krayenbuehl et al. 2010), hypertrophic cardiomyopathy (Daw et al. 2007), frontotemporal lobar degeneration (Finch et al. 2011) and amyloid polyneuropathy (Soares et al. 2005) among others. In familial late-onset Alzheimer disease, modifying loci may either influence the risk (Sleegers et al. 2009; Cruchaga et al. 2012) or the age of onset of disease (Wijsman et al. 2005; Marchani et al. 2010). Importantly, a significant excess of rare coding APP, PSEN1 and PSEN2 variants was noted in probands from late-onset Alzheimer disease families even though these variants did not actually cosegregate with the disease; this suggests that the variants in question may nevertheless serve to modulate the risk of disease (Cruchaga et al. 2012). An excess of rare variants as compared to controls has also been noted in patients with various other disorders including hypertriglyceridaemia (Johansen et al. 2012; Talmud 2007), hypertrophic 
cardiomyopathy (Lopes et al. 2013) and autism spectrum disorder (Mondal et al. 2012).

A typical example of a modifier gene in action is provided by long QT syndrome. The clinical penetrance of KCNQ1 (potassium voltage-gated channel, KQT-like subfamily, member 1) mutations in this disorder is influenced by two coding sequence polymorphisms [Ser49Gly (rs1801252) and Arg389Gly (rs1801253)] in the ADRB1 gene. Individuals homozygous for the Arg389 allele tend to have shorter QT intervals, whereas individuals homozygous for Ser49 tend to have longer QT intervals than those with other genotypes (Paavonen et al. 2007). Interestingly, those individuals doubly homozygous for Arg389 and Ser49 were found to be indistinguishable from the remainder of the patient cohort, both in terms of their QT intervals and in terms of clinical penetrance.

The minor allele of a variant in the complement receptor 1 (CR1) gene, Ser1610Thr (rs4844609), which has a population frequency of 0.02 , is associated with episodic memory decline and susceptibility to Alzheimer disease (Keenan et al. 2012). However, this effect appears largely dependent upon an interaction with APOE- $\varepsilon 4$, itself an important risk factor for Alzheimer disease (Mayeux et al. 1993).

Hirschsprung disease is one of the most complex genetic disorders in terms of the number of modifier genes (GarciaBarcelo et al. 2009; Tang et al. 2010) known to influence the penetrance of its causative mutations, which has been estimated to be of the order of 50-70\% (Bolk et al. 2000). The best characterized of these modifier genes is the neuregulin 1 gene (NRGI; Tang et al. 2011, 2012a); however, most probably still remain to be identified. Some BardetBiedl syndrome patients also present with Hirschsprung disease. It appears that $R E T$, the major gene involved in the aetiology of Hirschsprung disease, acts as a modifier of the Hirschsprung disease phenotype in Bardet-Biedl syndrome (de Pontual et al. 2007). Some families with Hirschsprung disease and Bardet-Biedl syndrome harbour mutations in their BBS4, BBS5, BBS7 and RET genes (de Pontual et al. 2009). Sánchez-Mejías et al. (2009) reported a Hirschsprung disease family in which mutations in three different genes (RET, NTRK3 and EDN3) contributed to the disease phenotype; the RET and NTRK3 mutations were both necessary and sufficient to give rise to the clinical phenotype, whereas the EDN3 mutation appeared to act as a modifier. More recently, copy number variations in various neurodevelopmental genes (MAPK10, ZFHX1B, SOX2 and $N R G 2$ ) have been shown to modify the penetrance of Hirschsprung disease (Jiang et al. 2011; Tang et al. 2012b). Taken together, these findings are consistent with an impact of both common and rare variants on the inheritance (and hence penetrance) of this highly complex disorder (Sánchez-Mejías et al. 2009; Núñez-Torres et al. 2011; Alves et al. 2013).
Table 3 lists a number of well-characterized examples of specific variants in modifier genes that serve to modulate the clinical penetrance of diseases caused by mutation(s) at unlinked loci.

The plastin 3 (PLS3) gene acts as a modifier of the clinical penetrance of autosomal recessive spinal muscular atrophy, caused by the homozygous deletion of the SMN1 gene. Oprea et al. (2008) studied spinal muscular atrophydiscordant families with affected and unaffected SMN1deleted siblings and found that all unaffected SMN1deleted siblings were characterized by a high PLS3 expression level in blood cells, considerably higher than in their affected counterparts (and despite high PLS expression being evident in only $5 \%$ of healthy controls). Although it is still unclear whether PLS3 expression is regulated by cis- or trans-acting factors, it would appear that high PLS3 expression serves to rescue the spinal muscular atrophy patient from the detrimental effects of SMN1 deletion by promoting axonogenesis through elevation of the level of F-actin (Oprea et al. 2008) and ultimately by improving neuromuscular transmission (Ackermann et al. 2013).

A unique kind of modifying effect is exemplified by $\alpha$ thalassaemia/mental retardation syndrome, caused by mutations of the ATRX gene. The ATRX protein binds to variable number tandem repeat sequences (VNTRs) in the human genome, and genes associated with these VNTRs are dysregulated when ATRX is mutated (Law et al. 2010). Law et al. (2010) identified 917 ATRX targets in primary human erythroid cells, including one in the $\alpha$-globin $(H B A)$ locus. This $\psi \delta$ VNTR was found to be highly polymorphic in terms of its length and acted as a length-dependent negative regulator of gene expression, its length serving to influence the degree of $\alpha$-thalassaemia observed in an $\alpha$ thalassaemia/mental retardation syndrome patient. Thus, the length of the $\psi \delta$ VNTR could explain the incomplete penetrance of $\alpha$-thalassaemia noted in individuals with identical ATRX mutations. A similar mechanism could underlie other genetic traits characterized by reduced penetrance.

\section{Digenic mutations and disease penetrance}

In a typical autosomal Mendelian condition, a single mutation (dominant) or two mutations (recessive) at a specific locus give rise to a clinical phenotype. By contrast, digenic inheritance occurs in cases where the interaction of mutations in two different genes is required for the expression of the clinical phenotype. In this situation, a mutation in one copy of each gene is required for the full clinical phenotype to manifest. In the absence of one of the component mutations, the other mutation may be non- 
Table 3 A selection of well-characterized examples of polymorphic variants in modifier genes that serve to modulate the clinical penetrance and/or severity of an inherited disease caused by mutation(s) at an unlinked locus

\begin{tabular}{|c|c|c|c|}
\hline Disease & Primary disease gene & Modifier gene/variant & Reference \\
\hline $\begin{array}{l}\mathrm{X} \text {-linked retinitis } \\
\text { pigmentosa }\end{array}$ & $R P G R$ & $\begin{array}{l}\text { IQCB1/NM_001023570.2:c.1178T >A Ile393Asn } \\
(\text { (rs1141528) }\end{array}$ & Fahim et al. (2011) \\
\hline $\begin{array}{l}\text { X-linked retinitis } \\
\text { pigmentosa }\end{array}$ & $R P G R$ & $\begin{array}{l}\text { RPGRIP1L/NM_015272.2:c.2231G >A Arg744Gln } \\
\quad(\text { rs2302677) }\end{array}$ & Fahim et al. (2011) \\
\hline $\begin{array}{l}\text { Retinal generation in } \\
\text { ciliopathies }\end{array}$ & $R P G R$ or $N P H P 5$ & $\begin{array}{l}\text { RPGRIP1L/NM_015272.2:c.685G >A Ala229Thr } \\
\quad(\text { rs61747071) }\end{array}$ & Khanna et al. (2009) \\
\hline Retinoblastoma & $R B 1$ & MDM2/NM_002392.4:c.14+309T>G (rs2279744) & Castéra et al. (2010) \\
\hline $\begin{array}{l}\text { Familial } \\
\text { hypercholesterolaemia }\end{array}$ & $L D L R$ & PCSK9/NM_174936.3:c.63_65dupGCT (rs35574083) & Abifadel et al. (2009) \\
\hline $\begin{array}{l}\text { Familial } \\
\text { hypercholesterolaemia }\end{array}$ & $L D L R$ & $\begin{array}{l}\text { APOB/NM_000384.2:c.10580G }>\text { A Arg3527Gln } \\
\text { (rs5742904) }\end{array}$ & $\begin{array}{l}\text { Benlian et al. (1996) } \\
\text { and Taylor et al. (2010) }\end{array}$ \\
\hline $\begin{array}{l}\text { Familial } \\
\text { hypercholesterolaemia }\end{array}$ & $L D L R$ & CFH/NM_000186.3:c.1204T>C Tyr402His (rs1061170) & Koeijvoets et al. (2009) \\
\hline $\begin{array}{l}\text { Familial } \\
\text { hypercholesterolaemia }\end{array}$ & $L D L R$ & APOH/NM_000042.2:c.1204T>C Leu266Val (rs4581) & Takada et al. (2003a) \\
\hline $\begin{array}{l}\text { Familial } \\
\text { hypercholesterolaemia }\end{array}$ & $L D L R$ & GHR/NM_000163.4:c.1630A>C Ile544Leu (rs6180) & Takada et al. (2003b) \\
\hline $\begin{array}{l}\text { Familial } \\
\text { hypercholesterolaemia }\end{array}$ & $L D L R$ & EPHX2/NM_001979.4:c.860G>A Arg287Gln (rs751141) & Sato et al. (2004) \\
\hline Breast cancer & $B R C A 2$ & RAD51/NM_002875.4:c.-98G >C (rs1801320) & Antoniou et al. (2007) \\
\hline Ovarian cancer & $B R C A 1$ or $B R C A 2$ & IRS1/NM_005544.2:c.2911G>A Gly971Arg (rs801278) & Ding et al. (2012) \\
\hline Lynch syndrome & $M S H 2$ or $M L H 1$ & $\begin{array}{l}\text { RNASEL/NM_021133.3:c.1385G >A Arg462Gln } \\
(\text { rs486907) }\end{array}$ & Krüger et al. (2007) \\
\hline Prostate cancer & $M S H 2$ or $M L H 1$ & $\begin{array}{l}\text { RNASEL/NM_021133.3:c.1385G >A Arg462Gln } \\
\text { (rs486907) }\end{array}$ & Krüger et al. (2005) \\
\hline Lynch syndrome & $M S H 2$ or $M L H 1$ & TP53/NM_000546.3:c.215G>C Arg72Pro (rs1042522) & Krüger et al. (2007) \\
\hline Cystic fibrosis & CFTR & TGFB1/NM_000660.4:c.29C>T Pro10Leu (rs1800470) & Drumm et al. (2005) \\
\hline $\begin{array}{l}\text { Familial pulmonary } \\
\text { arterial hypertension }\end{array}$ & $B M P R 2$ & $\begin{array}{l}\text { TGFB1/NM_000660.4:c.-1347C >T (rs1800469) \& } \\
\text { NM_000660.4:c.29C }>\text { T Leu10Pro (rs1800470) }\end{array}$ & Phillips et al. (2008) \\
\hline Paget's disease & SQSTM1 & $\begin{array}{l}\text { TNFRSF11A/NM_003839.2:c.575T >C Val192Ala } \\
\quad(\text { rs1805034) }\end{array}$ & Gianfrancesco et al. (2012) \\
\hline $\begin{array}{l}\text { X-linked variable } \\
\text { immunodeficiency }\end{array}$ & $X I A P$ & $\begin{array}{l}\text { CD40LG/NM_000074.2:c.655G >A Gly219Arg } \\
\text { (rs148594123) }\end{array}$ & Rigaud et al. (2011) \\
\hline Haemochromatosis & $H F E$ & CYBRD1/NM_024843.3:c.-399T>G (rs884409) & Constantine et al. (2009) \\
\hline Parkinson's disease & $G B A$ & MTX1/NM_002455.3:c.187T>A Ser63Thr (rs760077) & Gan-Or et al. (2011) \\
\hline $\begin{array}{l}\text { Recessive dystrophic } \\
\text { epidermolysis bullosa }\end{array}$ & COL7A1 & MMP1/NM_002421.3:c.-1673delG (rs 1799750) & Titeux et al. (2008) \\
\hline $\begin{array}{l}\text { Amyotrophic lateral } \\
\text { sclerosis }\end{array}$ & SOD1 & CHGB/NM_001819.2:c.1238C >T Pro413Leu (rs742710) & Gros-Louis et al. (2009) \\
\hline Huntington disease & HTT & HAP1/NM_177977.2:c.1322C $>$ T Thr441Met (rs4523977) & Metzger et al. (2008) \\
\hline Fatal kernicterus & $G 6 P D$ & $\begin{array}{l}U G T 1 A 1 /(\mathrm{TA})_{6} /(\mathrm{TA})_{7}(\mathrm{rs} 8175347), \text { HGVS nomenclature } \\
\text { not available }\end{array}$ & Zangen et al. (2009) \\
\hline $\begin{array}{l}\text { Atypical haemolytic } \\
\text { uraemic syndrome }\end{array}$ & $M C P$ or $C F H$ & $\begin{array}{l}\text { C4BPA/NM_000715.3:c.719G }>\text { A Arg240His } \\
\text { (rs45574833) }\end{array}$ & Blom et al. (2008) \\
\hline Spinal muscular atrophy & SMN1 & $\begin{array}{l}\text { SMN2/NM_017411.3:c.859G >C Gly287Arg } \\
\quad(\text { rs121909192) }\end{array}$ & Prior et al. (2009) \\
\hline Long QT syndrome & $K C N Q 1$ & $\begin{array}{l}\text { KCNH2/NM_000238.3:c.2690A >C Lys897Thr } \\
\text { (rs1805123) }\end{array}$ & Cordeiro et al. (2010) \\
\hline Long QT syndrome & $K C N Q 1$ & $\begin{array}{l}\text { ADRB1/NM_000684.2:c.145A }>\text { G Ser49Gly (rs1801252) \& } \\
\text { NM_000684.2:c.1165G }>\text { C Arg389Gly (rs1801253) }\end{array}$ & Paavonen et al. (2007) \\
\hline Long QT syndrome & $K C N Q 1$ & NOS1AP/NC_000001.10:g.162029907A > T (rs4657139) & Crotti et al. (2009) \\
\hline
\end{tabular}


Table 3 continued

\begin{tabular}{|c|c|c|c|}
\hline Disease & Primary disease gene & Modifier gene/variant & Reference \\
\hline $\begin{array}{l}\text { Familial venous } \\
\text { thrombosis }\end{array}$ & PROC & $\begin{array}{l}\text { F5/NM_000130.4:c.1601G >A Arg534Gln (rs6025; } \\
\text { Factor V Leiden) }\end{array}$ & $\begin{array}{l}\text { Koeleman et al. (1994); } \\
\text { Gandrille et al. (1995) } \\
\text { and Cafolla et al. (2012) }\end{array}$ \\
\hline $\begin{array}{l}\text { Familial venous } \\
\text { thrombosis }\end{array}$ & PROS1 & $\begin{array}{l}\text { F5/NM_000130.4:c.1601G >A Arg534Gln (rs6025; } \\
\text { Factor V Leiden) }\end{array}$ & Koeleman et al. (1995) \\
\hline $\begin{array}{l}\text { Familial venous } \\
\text { thrombosis }\end{array}$ & SERPINC1 & $\begin{array}{l}\text { F5/NM_000130.4:c.1601G }>\text { A Arg534Gln (rs6025; } \\
\text { Factor V Leiden) }\end{array}$ & Van Boven et al. (1996) \\
\hline $\begin{array}{l}\text { Hypertrophic } \\
\text { cardiomyopathy }\end{array}$ & $M Y B P C 3$ or $M Y H 7$ & CALM3/NM_005184.2:c.-157T>A (rs150954567) & Friedrich et al. (2009) \\
\hline $\begin{array}{l}\text { Familial Mediterranean } \\
\text { fever }\end{array}$ & $M E F V$ & SAA1/NM_000331.4:c.-197C>T (rs11024595) & Migita et al. (2013) \\
\hline
\end{tabular}

penetrant (digenic inheritance sensu stricto) or could be responsible for a less severe clinical phenotype (digenic inheritance sensu lato). At least 100 cases of probable and possible examples of digenic inheritance causing human inherited disease have been reported to date (Table 4).

Digenic inheritance may occur as a result of mutations in genes encoding different subunits of the same multimeric protein (e.g. $P R P H 2$ and $R O M 1$ ), an oligomeric protein complex (e.g. KCNJ10 and SLC26A4; KRT14 and $K R T 5)$ or simply two proteins that interact functionally with each other (CDH23 and PCDH15; DSG2 and DSC2; PARK7 and PINK1). However, mutations in receptor/ ligand pairs can also give rise to digenic inheritance (e.g. $P R O K 2$ and PROKR2). Alternatively, digenic inheritance can involve mutations located in different genes, but compromising the same regulatory (e.g. $H F E$ and $H A M P$ ), biosynthetic (e.g. ZMPSTE24 and LMNA; CPOX and PPOX; MYOC and CYPIB1) or degradative (e.g. PCSK9 and $L D L R$ ) pathway. Finally, the combination of a mutation in a transcription factor with a mutation in a target gene of that transcription factor can also serve to reduce the amount of the protein in question to a level sufficient to cause a disease phenotype (e.g. MITF and TYR; FOXC1 and PITX2).

In practice, it is not always clear if a given situation constitutes true digenic inheritance (e.g. Kajiwara et al. 1994) or whether it is simply the coinheritance of two mutations in different genes (e.g. Gruber et al. 2009; Serrano-Fernández et al. 2009; Ekvall et al. 2011). In the former case, the expression of the disease phenotype actually requires the presence of both gene lesions. In the latter case, coinheritance of the two gene lesions may serve to aggravate the clinical phenotype, but each lesion is independently associated with its own characteristic clinical sequelae. In true digenic inheritance, mutations in both genes must be present for the genetic disorder to be manifest. Since many of the disorders reported (in Table 4) to be characterized by digenic inheritance also have monogenic forms in which just one of the two genes has been mutated (whether in the heterozygous or homozygous/ compound heterozygous state), it is unclear how many of the examples listed really represent digenic inheritance sensu stricto. However, in many of the listed examples, the doubly heterozygous probands exhibit earlier onset or a more severe clinical phenotype than their singly heterozygous relatives (although this is not invariably so; Marras et al. 2010). Further, whereas the truly 'digenic' patients tend to be characterized by complete penetrance, the monogenic disease genotypes often exhibit reduced penetrance as in e.g. normosmic idiopathic hypogonadotrophic hypogonadism (see Table 4).

In some of the cases listed in Table 4, the digenic inheritance may be confined to a single proband and hence it is not always straightforward to distinguish true digenic inheritance from the chance coinheritance of two mutations in unlinked genes. This notwithstanding, the requirement for the involvement of a second mutated gene may depend upon the specific mutations that are segregating in the pedigrees. If digenic inheritance eventually turns out to be more frequent than previously appreciated, it could provide yet another reason why some potentially pathogenic alleles are present in the general population in the absence of overt disease. At the very least, in many cases the clinical penetrance of the condition in question is likely to be greater when two relevant genes have been functionally compromised by mutation than if only one had been mutated.

\section{Oligogenic inheritance and its implications for disease penetrance}

Triallelic inheritance has been described as being "a bridge between Mendelian and multifactorial traits" (Eichers et al. 2004). There are a burgeoning number of reported examples of digenic triallelic inheritance including nephronophthisis (Hoefele et al. 2007), venous thrombosis (Formstone et al. 1996; Brenner et al. 1996) and cortisone 
Table 4 Examples of digenic mutations causing human inherited disease

\begin{tabular}{|c|c|c|c|}
\hline Disease & Gene 1 & Gene 2 & Reference \\
\hline Waardenburg syndrome type $2 *$ & MITF & $\begin{array}{l}\text { PAX3 or } O C A 3 \text { or } T Y R \text { or } \\
\quad \text { GJB2 }\end{array}$ & $\begin{array}{l}\text { Morell et al. (1997); Chiang et al. (2009); Yan } \\
\text { et al. (2011) and Yang et al. (2013) }\end{array}$ \\
\hline Retinitis pigmentosa* & PRPH2 & ROM1 or $R H O$ or $P D E 6 B$ & $\begin{array}{l}\text { Kajiwara et al. (1994); Loewen et al. (2001); } \\
\text { Sullivan et al. (2006) and Jin et al. (2008) }\end{array}$ \\
\hline Retinitis pigmentosa & $\mathrm{RHO}$ & PRPF31 & Lim et al. (2009) \\
\hline Retinitis pigmentosa* & $P D E 6 B$ & GPR98 & Hmani-Aifa et al. (2009) \\
\hline Progressive cone dystrophy & $C N G A 3$ & $C N G B 3$ & Thiadens et al. (2010) \\
\hline Frontotemporal dementia* & PSEN1 & $P R N P$ & Bernardi et al. (2011) \\
\hline Leber congenital amaurosis* & RPE65 & $G U C Y 2 D$ & Silva et al. (2004) \\
\hline Idiopathic hypogonadotropic hypogonadism* & FGFR1 & GNRHR or NELF & Pitteloud et al. (2007) \\
\hline Bilateral cystic renal dysplasia & $\mathrm{DACH} 1$ & BMP4 & Schild et al. (2013) \\
\hline Glaucoma, early onset* & MYOC & $C Y P 1 B 1$ or $L T B P 2$ & $\begin{array}{l}\text { Vincent et al. (2002), Geyer et al. (2011) and } \\
\text { Azmanov et al. (2011) }\end{array}$ \\
\hline Severe insulin resistance & PPARG & $P P P 1 R 3 A$ & Savage et al. (2002) \\
\hline Usher syndrome type $2^{*}$ & PDZD7 & GPR98 & Ebermann et al. (2010) \\
\hline Usher syndrome type 1-associated deafness & $\mathrm{CDH} 23$ & PCDH15 & Zheng et al. (2005) \\
\hline Hidrotic ectodermal dysplasia & GJB2 & GJA1 & Kellermayer et al. (2005) \\
\hline Non-syndromic deafness* & GJB2 & GJB3 & Liu et al. (2009) \\
\hline Hearing loss & $G J B 2$ & SLC26A4 & Sagong et al. (2013) \\
\hline $\begin{array}{l}\text { Non-syndromic hearing loss associated with an } \\
\text { enlarged vestibular aqueduct/Pendred } \\
\text { syndrome }\end{array}$ & KCNJ10 & $S L C 26 A 4$ & Yang et al. (2009) \\
\hline Porphyria* & CPOX & $P P O X$ & van Tuyll van Serooskerken et al. (2011) \\
\hline Atypical haemolytic uremic syndrome & $C F I$ & $\begin{array}{l}C D 46 \text { or } C 3 \text { or } C F B \text { or } \\
\text { CFHR1 }\end{array}$ & $\begin{array}{l}\text { Esparza-Gordillo et al. (2006), Westra et al. } \\
\text { (2010) and Bresin et al. (2013) }\end{array}$ \\
\hline Atypical haemolytic uraemic syndrome & $\mathrm{CFH}$ & $\begin{array}{l}C D 46 \text { or } C F I \text { or } C 3 \text { or } \\
\text { THBD }\end{array}$ & $\begin{array}{l}\text { Sullivan et al. (2011), Bresin et al. (2013) and } \\
\text { Fan et al. (2013) }\end{array}$ \\
\hline Epidermolysis bullosa simplex* & KRT14 & KRT5 & Padalon-Brauch et al. (2012) \\
\hline Junctional epidermolysis bullosa & COL17A1 & $L A M B 3$ & Floeth and Bruckner-Tuderman (1999) \\
\hline Long QT syndrome* & $K C N Q 1$ & $\begin{array}{l}\text { KCNH2 or KCNE1 or } \\
\text { SCN5A }\end{array}$ & $\begin{array}{l}\text { Schwartz et al. (2003), Westenskow et al. (2004), } \\
\text { Tester et al. (2005) and Itoh et al. (2010) }\end{array}$ \\
\hline Long QT syndrome* & $\mathrm{KCNH} 2$ & $S C N 5 A$ or $K C N E 1$ & $\begin{array}{l}\text { Schwartz et al. (2003), Westenskow et al. (2004) } \\
\text { and Tester et al. (2005) }\end{array}$ \\
\hline Long QT syndrome* & $S C N 5 A$ & SNTA1 or $K C N E 1$ & Westenskow et al. (2004) and Hu et al. (2013) \\
\hline Haemochromatosis* & $H F E$ & $H A M P$ or $T F R 2$ & $\begin{array}{l}\text { Merryweather-Clarke et al. (2003), Jacolot et al. } \\
\text { (2004), Island et al. (2009), Altès et al. (2009) } \\
\text { and Del-Castillo-Rueda et al. (2012) }\end{array}$ \\
\hline Kallmann syndrome* & PROK2 & PROKR2 & $\begin{array}{l}\text { Cole et al. (2008), Sarfati et al. (2010) and Shaw } \\
\text { et al. (2011) }\end{array}$ \\
\hline Kallmann syndrome & $N E L F$ & $K A L 1$ or $T A C R 3$ & Xu et al. (2011) and Quaynor et al. (2011) \\
\hline Kallmann syndrome & PROKR2 & $K A L 1$ & $\begin{array}{l}\text { Dodé et al. (2006), Canto et al. (2009) and Shaw } \\
\text { et al. (2011) }\end{array}$ \\
\hline Kallmann syndrome & $K A L 1$ & $\begin{array}{l}\text { TACR } 3 \text { or WDR } 11 \text { or } \\
\text { CHD } 7\end{array}$ & Quaynor et al. (2011) and Shaw et al. (2011) \\
\hline $\begin{array}{l}\text { Normosmic idiopathic hypogonadotrophic } \\
\text { hypogonadism }\end{array}$ & GNRH & $K A L 1$ & Quaynor et al. (2011) \\
\hline $\begin{array}{l}\text { Normosmic idiopathic hypogonadotrophic } \\
\text { hypogonadism }\end{array}$ & WDR11 & GNRHR & Quaynor et al. (2011) \\
\hline $\begin{array}{l}\text { Normosmic idiopathic hypogonadotrophic } \\
\text { hypogonadism }\end{array}$ & FGFRl & $\begin{array}{l}\text { GNRHR or } P R O K R 2 \text { or } \\
F G F 8 \text { or } K A L 1 \text { or } G P R 54\end{array}$ & $\begin{array}{l}\text { Raivio et al. (2009), Sykiotis et al. (2010) and } \\
\text { Shaw et al. (2011) }\end{array}$ \\
\hline Systemic amyloid A amyloidosis & TNFRSF1A & $M E F V$ & Cigni et al. (2006) and Mereuta et al. (2013) \\
\hline
\end{tabular}


Table 4 continued

\begin{tabular}{|c|c|c|c|}
\hline Disease & Gene 1 & Gene 2 & Reference \\
\hline Familial hypercholesterolaemia* & $L D L R$ & PCSK9 & $\begin{array}{l}\text { Pisciotta et al. (2006), Noguchi et al. (2010) and } \\
\text { Bertolini et al. (2013) }\end{array}$ \\
\hline Familial hypercholesterolaemia & $L D L R$ & $A P O B$ & Bertolini et al. (2013) \\
\hline Familial hypercholesterolaemia & $L D L R$ & LDLRAP1 & Tada et al. (2011) \\
\hline Severe congenital neutropenia & ELANE & G6PC3 or $H A X 1$ & Germeshausen et al. (2010) \\
\hline McArdle's disease* & $P Y G M$ & $C P T 2$ & Vockley et al. (2000) \\
\hline Parkinson's disease, early onset & PINK1 & $P A R K 2$ or $P A R K 7$ & Tang et al. (2006) and Funayama et al. (2008) \\
\hline Parkinson's disease & $L R R K 2$ & $P R K N$ & Dächsel et al. (2006) \\
\hline Emery-Dreifuss muscular dystrophy* & $L M N A$ & $D E S$ & Muntoni et al. (2006) \\
\hline Joubert syndrome and nephronophthisis* & NPHP1 & NPHP6 & Tory et al. (2007) \\
\hline Axenfeld-Rieger syndrome & FOXC1 & PITX2 & Kelberman et al. (2011) \\
\hline Cortisone reductase deficiency & $H S D 11 B 1$ & $H 6 P D$ & Draper et al. (2003) and San Millán et al. (2005) \\
\hline Hypertrophic cardiomyopathy* & $M Y B P C 3$ & $\begin{array}{l}\text { TNNT2 or TNNI3 or } M Y H 7 \\
\text { or TPM1 }\end{array}$ & $\begin{array}{l}\text { Richard et al. (2003), Van Driest et al. (2004) } \\
\text { Ingles et al. (2005), Millat et al. (2010), Kubo } \\
\text { et al. (2011) and Zou et al. (2013) }\end{array}$ \\
\hline Hypertrophic cardiomyopathy* & MYH7 & $\begin{array}{l}\text { TNNT2 or MYL2 or TNNI3 } \\
\text { or ACTC1 }\end{array}$ & Millat et al. (2010) and Zou et al. (2013) \\
\hline Restrictive cardiomyopathy* & MYL2 & MYL3 & Caleshu et al. (2011) \\
\hline $\begin{array}{l}\text { Rasopathy phenotype with severe hypertrophic } \\
\text { cardiomyopathy }\end{array}$ & PTPN11 & SOS1 & Fahrner et al. (2012) \\
\hline $\begin{array}{l}\text { Arrhythmogenic right ventricular } \\
\text { cardiomyopathy }\end{array}$ & $D E S$ & $P K P 2$ & Lorenzon et al. (2013) \\
\hline $\begin{array}{l}\text { Arrhythmogenic right ventricular } \\
\text { cardiomyopathy* }\end{array}$ & $D E S$ & $D S G 2$ & Rasmussen et al. (2013) \\
\hline $\begin{array}{l}\text { Arrhythmogenic right ventricular } \\
\text { cardiomyopathy }\end{array}$ & $P K P 2$ & $\begin{array}{l}D S P \text { or } D S G 2 \text { or } P K P 4 \text { or } \\
\quad D S C 2\end{array}$ & Xu et al. (2010) \\
\hline $\begin{array}{l}\text { Arrhythmogenic right ventricular dysplasia/ } \\
\text { cardiomyopathy }\end{array}$ & $D S G 2$ & $D S C$ or $P K P 2$ & Bhuiyan et al. (2009) and Nakajima et al. (2012) \\
\hline Familial dilated cardiomyopathy* & $L M N A$ & $T T N$ & Roncarati et al. (2013) \\
\hline Dent's disease & CLCN5 & $O C R L$ & Addis et al. (2013) \\
\hline Amyotrophic lateral sclerosis & SOD1 & CNTF & Giess et al. (2002) \\
\hline Amyotrophic lateral sclerosis & $V A P B$ & C9orf72 & van Blitterswijk et al. (2012b) \\
\hline Dravet syndrome & РCDH19 & TSPYL4 & Kwong et al. (2012) \\
\hline Dravet syndrome* & SCN9A & SCN1A & Singh et al. (2009) \\
\hline Dravet syndrome* & CACNA1A & SCN1A & Ohmori et al. (2013) \\
\hline Severe myoclonic epilepsy & CACNB4 & SCN1A & Ohmori et al. (2008) \\
\hline Severe myoclonic epilepsy & $P O L G$ & SCN1A & Bolszak et al. (2009) \\
\hline Progressive external ophthalmoplegia & $P O L G$ & SLC25A4 & Galassi et al. (2008) \\
\hline Bartter syndrome & $C L C N K A$ & $C L C N K B$ & Nozu et al. (2008) \\
\hline Chronic pancreatitis & SPINK1 & $\begin{array}{l}\text { CASR or } C F T R \text { or } C T R C \text { or } \\
\text { PRSS1 }\end{array}$ & $\begin{array}{l}\text { Felderbauer et al. (2003), Masson et al. (2007), } \\
\text { Tzetis et al. (2007), Schneider et al. (2011), } \\
\text { LaRusch et al. (2012) and Rosendahl et al. } \\
\text { (2013) }\end{array}$ \\
\hline Oculocutaneous albinsim & $O C A 2$ & $T Y R P 1$ or $S L C 45 A 2$ or $T Y R$ & Chiang et al. (2008) and Wei et al. (2013) \\
\hline Oculocutaneous albinsim & $T Y R$ & $S L C 45 A 2$ & Wei et al. (2013) \\
\hline Cystinuria & SLC $3 A 1$ & SLC7A9 & Font-Llitjós et al. (2005) \\
\hline Transposition of the great arteries & ZIC3 & FOXH1 or $N K X 2-5$ & De Luca et al. (2010) \\
\hline Congenital heart disease & МYH6 & NKX2-5 or GATA4 & Granados-Riveron et al. (2012) \\
\hline Charcot-Marie-Tooth disease* & $P M P 22$ & $A B C D 1$ or $L I T A F$ & Meggouh et al. (2005), Hodapp et al. (2006) \\
\hline Charcot-Marie-Tooth disease & GJB1 & EGR2 & Chung et al. (2005) \\
\hline Charcot-Marie-Tooth disease* & $G D A P 1$ & MFN2 & Vital et al. (2012) \\
\hline
\end{tabular}


Table 4 continued

\begin{tabular}{|c|c|c|c|}
\hline Disease & Gene 1 & Gene 2 & Reference \\
\hline Refractory auto-inflammatory syndrome & TNFRSF1A & CIAS1 & Touitou et al. (2006) \\
\hline Short-rib polydactyly syndrome type 2 & NEK1 & $D Y N C 2 H 1$ & Thiel et al. (2011) \\
\hline Maturity-onset diabetes of the young & $H N F 1 A$ & $H N F 1 B$ & Karges et al. (2007) \\
\hline Maturity-onset diabetes of the young & HNF1A & $H N F 4 A$ & Forlani et al. (2010) and Shankar et al. (2013) \\
\hline Polycystic kidney disease* & PKD1 & $P K D 2$ & Pei et al. (2001) and Dedoussis et al. (2008) \\
\hline $\begin{array}{l}\text { Hyperimmunoglobulinaemia D and periodic } \\
\text { fever syndrome }\end{array}$ & $M V K$ & TNFRSF1A & Hoffmann et al. (2005) \\
\hline $\begin{array}{l}\text { Obesity, hyperinsulinaemia and insulin } \\
\text { resistance }\end{array}$ & $T C F 1$ & $N R O B 2$ & Tonooka et al. (2002) \\
\hline Progressive external ophthalmoplegia & $P O L G$ & C10orf2 & Van Goethem et al. (2003) \\
\hline Neuronal ceroid lipofuscinosis & $P O L G$ & CLN5 & Staropoli et al. (2012) \\
\hline Chronic lung disease & SFTPC & $A B C A 3$ & Bullard and Nogee (2007) \\
\hline Lafora disease & $E P M 2 B$ & $P P P 1 R 3 C$ & Guerrero et al. (2011) \\
\hline Congenital erythropoietic porphyria & UROS & ALAS2 & To-Figueras et al. (2011) \\
\hline Familial venous thrombosis* & PROC & PROS1 & $\begin{array}{l}\text { Formstone et al. (1996), Brenner et al. (1996), } \\
\text { Boinot et al. (2003), Knoll et al. (2001) and } \\
\text { Hayashida et al. (2003) }\end{array}$ \\
\hline Familial venous thrombosis* & PROC & SERPIND1 & Bernardi et al. (1996) \\
\hline Breast cancer* & $B R C A 1$ & $B R C A 2$ & $\begin{array}{l}\text { Leegte et al. (2005), Lavie et al. (2011) and } \\
\text { Heidemann et al. (2012) }\end{array}$ \\
\hline Breast cancer & $B R C A 1$ & $P A L B 2$ & Pern et al. (2012) \\
\hline Multiple tumours of different types & $B R C A 1$ & $M L H 1$ & Pedroni et al. (2013) \\
\hline Familial pulmonary arterial hypertension & $B M P R 2$ & THBS1 & Maloney et al. (2012) \\
\hline Hereditary nonpolyposis colorectal cancer & MUTYH & MSH6 & $\begin{array}{l}\text { Van Puijenbroek et al. (2007) and Giráldez et al. } \\
\text { (2009) }\end{array}$ \\
\hline Colorectal cancer & EPCAM & $\mathrm{MSH} 2$ & Li-Chang et al. (2013) \\
\hline Colorectal cancer, juvenile onset* & $A P C$ & MSH2 & Uhrhammer and Bignon (2008) \\
\hline Autoimmune lymphoproliferative syndrome & $F A S$ & CASP10 & Cerutti et al. (2007) \\
\hline Autoimmune lymphoproliferative syndrome* & FAS & PRF1 & Clementi et al. (2004) \\
\hline $\begin{array}{l}\text { Steroid-resistant focal segmental } \\
\text { glomerulosclerosis }\end{array}$ & NPHS2 & NPHS1 or $C D 2 A P$ & Löwik et al. (2008) \\
\hline Severe infantile liver disease & $A K R 1 D 1$ & $S K I V 2 L$ & Morgan et al. (2013) \\
\hline Ataxia, dementia and hypogonadotropism & $R N F 216$ & OTUD4 & Margolin et al. (2013) \\
\hline Paediatric inflammatory bowel disease & NOD2 & $\begin{array}{l}G S D M B \text { or } Z N F 365 \text { or } \\
E R A P 2 \text { or } S E C 16 A \text { or } \\
G M P B B\end{array}$ & Christodoulou et al. (2013) \\
\hline Paediatric inflammatory bowel disease & $\mathrm{BACH} 2$ & IL10 & Christodoulou et al. (2013) \\
\hline Hutchinson-Gilford progeria syndrome & ZMPSTE24 & $L M N A$ & Denecke et al. (2006) \\
\hline
\end{tabular}

In this table, we considered only those examples of digenic mutations that are unlikely to be merely coincidental and which are predicted to affect genes that are both functionally associated with the disease in question

* Patients, with heterozygous mutations affecting two different genes, exhibiting earlier disease onset or a more severe clinical phenotype than either of their singly heterozygous parents or their siblings

reductase deficiency (Draper et al. 2003). It would not be altogether surprising, in conditions where digenic or even trigenic inheritance has been reported, if the individual component mutations were found to exhibit a reduced clinical penetrance as compared to mutations underlying the monogenic forms of the disease.

In some disorders, incomplete penetrance of a particular mutation can be due to the oligogenic nature of the disease and hence to the requirement for multiple genes to be mutated for the condition in question to manifest. An inherited predisposition to cancer can be monogenic, but is also very likely to have an oligogenic aetiology in many instances (Fearnhead et al. 2004; Koren-Michowitz et al. 2005; Okkels et al. 2006; Küry et al. 2008; Wasielewski et al. 2010; Martinez and Kolodner 2010; Plon et al. 2011; Morak et al. 2011; Gracia-Aznarez et al. 2013). In 
amyotrophic lateral sclerosis, van Blitterswijk et al. (2012a) detected FUS and TARDBP mutations in combination with $A N G$ mutations, and $C 9$ orf 72 repeat expansions with TARDBP, SODI and FUS mutations. At least five relatively common polymorphisms in four different genes, $C F B$ (Arg32Gln, rs641153), C2 (Glu318Asp, rs9332739), CFH [Tyr402His (rs1061170) and non-coding variant rs1410996] and ARMS2 (Ala69Ser, rs10490924), interact so as to confer increased risk of age-related macular degeneration (Maller et al. 2006). Common polymorphisms in the LEPR (Gln223Arg, rs1137101) and ADRB2 [Arg16Gly (rs1042713) and Gln27Glu (rs1042714)] genes jointly confer increased risk of obesity even though none of these polymorphisms exhibits a significant influence on their own (Pereira et al. 2011). Other examples of oligogenic inheritance, involving the mutation or polymorphism of multiple unlinked genes in the same individual, include isolated gonadotropin-releasing hormone deficiency (KAL1, PROK2 and NELF; Sykiotis et al. 2010), hypertrophic cardiomyopathy (MYH7, MYBPC3, TNNI3 and TNNT2; Girolami et al. 2010; Lopes et al. 2013), iminoglycinuria (SLC36A2, SLC6A20, SLC6A18, SLC6A19; Bröer et al. 2008), long QT syndrome ( $K C N H 2$, SCN5A and $K C N E 1$; Yoshikane et al. 2013), chronic pancreatitis (SPINK1, CFTR and CTRC; Rosendahl et al. 2013), atypical haemolytic uraemic syndrome (CFH, CD46 and CFI; Roumenina et al. 2012; Bresin et al. 2013), Parkinson disease (LRRK2, SNCA, MAPT, GBA, BST1, PARK16; Wang et al. 2012), acrocallosal syndrome (KIF7, AHI1, BBS2 and BBS4; Walsh et al. 2013), autism spectrum disorders (Neale et al. 2012) and a low plasma level of HDL cholesterol, a major risk factor for atherosclerosis (Cohen et al. 2004; Wang et al. 2008; Johansen and Hegele 2012).

One of the best characterized oligogenic disorders is Bardet-Biedl syndrome (BBS) where at least 17 genes are known to contribute to the clinical phenotype, and the severity of the disease phenotype may vary as a result of the interaction of mutations in different BBS genes. Although mutations at more than one locus have often been found to segregate with the disease, thereby modulating both its penetrance and expressivity (Badano et al. 2006; Zaghloul and Katsanis 2009; Cardenas-Rodriguez et al. 2013), the jury is still out in relation to claims of digenic triallelic inheritance in BBS (Katsanis et al. 2001; Beales et al. 2003; Badano et al. 2003; Fauser et al. 2003; Smaoui et al. 2006; Chen et al. 2011; Abu-Safieh et al. 2012). This notwithstanding, some common BBS gene variants appear to be detrimental to protein function and may well interact with the much rarer pathogenic BBS mutations so as to influence the severity of the BBS phenotype (Zaghloul et al. 2010).

Some idea of the likely complexity of genotype-phenotype relationships in complex disease has come from the comparative exome sequencing of 237 ion channel genes performed in sporadic idiopathic epilepsy patients and unaffected controls (Klassen et al. 2011). Both rare and common variants were identified in the two groups. Although these variants were more numerous in the patient group, they were not found to be predictive of disease: as the authors opined, "absolute numerical counts of SNP burden hold little predictive value as a global pathogenic measure". However, $51 \%$ of cases and $14 \%$ of controls had $\geq 2$ non-synonymous variants in their sodium channel genes. In similar vein, $24 \%$ of cases and $6 \%$ of controls had $\geq 2$ non-synonymous variants in their GABA receptor alpha genes (Klassen et al. 2011). Such findings are quite consistent with an oligogenic model of sporadic epilepsy (Dibbens et al. 2007). What is required here, however, is not a simple variant number count, but rather an assessment, on an individual basis, of the net effect of an oligogenic variant profile (requiring ascertainment of the various gains or losses of function associated with specific variants and computed with an eye to the nature of potential joint effects) on a clinically or phenotypically relevant output measure such as the electrical signature of a cell type or brain region.

Perhaps, the best characterized oligogenic disorder to date is familial venous thrombosis. The risk of venous thromboembolism is known to be increased in patients who carry more than one genetic variant disrupting the $100+$ genes of the 'hemostaseome' (Fechtel et al. 2011). Thus, $19 \%$ of symptomatic individuals harbouring a protein $\mathrm{C}$ (PROC) gene mutation were also found to be heterozygous for factor V Leiden (F5 Arg534Gln; Koeleman et al. 1994), a functional polymorphism which occurs at a frequency of $2-5 \%$ in European populations. In a replication study, $9.5 \%$ of venous thrombosis patients were found to carry both mutations (Gandrille et al. 1995) suggesting that their co-occurrence increases the likelihood of their coming to clinical attention. Similar findings have been noted in families with protein $\mathrm{S}$ (PROS1) deficiency; among symptomatic individuals, $38 \%$ also carried the factor $\mathrm{V}$ Leiden mutation (Koeleman et al. 1995). Likewise, coinheritance of antithrombin (SERPINC1) deficiency and factor V Leiden not only increases clinical penetrance, but also reduces the age of clinical presentation (van Boven et al. 1996). In a larger-scale study involving 132 thrombophilic families, the risk of thrombosis was increased and the age of onset lowered in cases of double heterozygosity for two gene variants (combinations of variants in PROC, PROS1, F5 and F2) as compared to individuals carrying single variants of these genes (Tirado et al. 2001). ABO blood group is also known to modify the risk of venous thrombosis in individuals with hereditary thrombophilia through an influence on the plasma levels of factor VIII and the factor VIII carrier protein, von Willebrand factor (Tirado et al. 2005; Nossent et al. 2006; Cohen et al. 2012). Such studies provide strong circumstantial 
support for the joint impact of multiple mutations in thrombotic disease (Martinelli et al. 2008). The elevation of risk for each individual variant is, however, low, and incomplete penetrance is evident for all prothrombotic variants. This means that the vast majority of individuals bearing these variants do not suffer from thrombotic disease. It is nevertheless reasonable to suppose that patients with recurrent venous thrombosis will tend to have a greater number of prothrombotic variants than those who have experienced a single thrombotic event, with those individuals who never experienced thrombosis harbouring even fewer prothrombotic variants (Fechtel et al. 2011). Since the number of genes known to influence haemostasis is large and the number of variants with potential impact larger still, we may expect that a substantial number of different variant combinations will be capable of conferring an increased risk. This genetic risk will accompany each prothrombotic challenge (such as pregnancy, long haul air travel, contraceptive pill usage and immobilization after surgery), with the greatest risk of venous thrombosis accruing to those possessing the largest number of prothrombotic variants in their genomes (Fechtel et al. 2011). Our task is to come to understand how specific DNA sequence changes in the large number of genes known to play a role in haemostasis and thrombosis act either synergistically or antagonistically so as to confer disease predisposition upon the individual, thereby influencing the clinical penetrance, by shifting their haemostatic balance towards either a prothrombotic or anticoagulant phenotype (Franchini and Mannucci 2009; Westrick and Ginsburg 2009; Fechtel et al. 2011).

\section{Influence of sex on penetrance}

The sex dependence of the penetrance of inherited mutations has been reported in a variety of different heritable disorders including haemochromatosis (HFE; Rossi et al. 2008), hypertrophic cardiomyopathy (MYBPC3, MYH7; Michels et al. 2009; Page et al. 2012), arrhythmogenic right ventricular dysplasia/cardiomyopathy ( $P K P 2$; Dalal et al. 2006), long QT syndrome (KCNQ1, KCNH2, SCN5A; Zareba et al. 2003), hypokalaemic periodic paralysis (CACNA1S; Kawamura et al. 2004; Li et al. 2012; SCN4A; Ke et al. 2013), familial pulmonary arterial hypertension (BMPR2; Austin et al. 2009b), hereditary spastic paraplegia (SPAST; Mitne-Neto et al. 2007), hereditary dystonia/dopa-responsive dystonia ( $G C H 1$; Furukawa et al. 1998), cardiac disease (LMNA; van Rijsingen et al. 2013), Hirschsprung disease (RET; Emison et al. 2005), autism spectrum disorder (SHANK1; Sato et al. 2012), amyotrophic lateral sclerosis (C9ORF72; Le Ber et al. 2013; Williams et al. 2013) and familial obesity (SHP; Yang et al. 2010). A male-biased effect on the penetrance of duplications and deletions at $16 \mathrm{p} 13.11$ is evident in a range of neorodevelopmental conditions including autism, attention deficit hyperactivity disorder, intellectual disability and schizophrenia (Tropeano et al. 2013). In the case of familial pulmonary arterial hypertension, both genetic and metabolic marker data were consistent with a modifying role for variation in oestrogens and/or oestrogen metabolism upon disease risk (Austin et al. 2009b). The low penetrance of hypokalaemic periodic paralysis due to $S C N 4 A$ mutations in females is also likely to be due to the effect of oestrogens (Ke et al. 2013).

Allelic variation may also influence the clinical phenotype in a sex-specific fashion. Thus, Lahtinen et al. (2011) reported that the common KCNE1 Asp85Asn (rs1805128) polymorphism was associated with a QT-interval prolongation in male but not female type 1 long QT syndrome patients harbouring the KCNQ1 Gly589Asp mutation. KCNE1 Asp85Asn may thus be a sex-specific QT-interval modifier in type 1 LQTS. Similarly, the Ile148Met (rs738409) PNPLA3 polymorphism is a disease modifier in primary sclerosing cholangitis with bile duct stenosis, but only in male patients (Friedrich et al. 2013). Finally, a Val89Leu polymorphism (rs523349) in the steroid $5 \alpha$-reductase type 2 (SRD5A2) gene, which serves to reduce the conversion of testosterone to dihydrotestosterone, has been claimed to influence the severity of post-traumatic stress symptoms but in a male-specific fashion (Gillespie et al. 2013).

An intriguing parent-of-origin effect has been noted in two apparently unrelated retinoblastoma families with a heterozygous, low-penetrance splice site mutation (c.607+1G $>\mathrm{T})$ in the $R B 1$ gene which causes skipping of exon 6 (Klutz et al. 2002). The abundance of the resulting nonsense (frameshifted) $R B 1 \mathrm{mRNA}$ relative to the wildtype was found to vary between members of one and the same family. Those individuals in family \#1 who inherited the mutant $R B 1$ allele from their mother displayed a similar level of nonsense and wild-type $R B I$ transcripts, and only one of eight carriers developed retinoblastoma. By contrast, those individuals in family \#2 who inherited the mutant $R B 1$ allele from their fathers displayed a reduced abundance of the nonsense transcript with six of eight carriers developing retinoblastoma, indicating that the mutant transcript has residual function. Assuming that this is not a chance result (Fisher's exact test; $p=0.04$ ), it may be that the gender of the transmitting parent can influence the penetrance of the pathogenic mutation.

There is good evidence to suggest that sex-specific genomic architecture can influence the expression of human phenotypes, including disease traits (Ober et al. 2008). It is likely that the underlying mechanism is differential gene regulation in males and females, particularly in relation to sex steroid-responsive genes (Zhang et al. 2007; Dimas et al. 2012).

Another mechanism by which sex influences penetrance is via genomic imprinting. Genomic imprinting results 
from the epigenetic modification of a gene or gene region that leads to the mutually exclusive expression of either the maternal or the paternal allele. Imprinted alleles are silenced (by DNA methylation or histone modification), so that the corresponding genes are expressed only from the non-imprinted allele inherited from the other parent. In the case of disease genes, imprinting can influence the penetrance of pathological mutations depending upon whether the wild-type or the mutant allele is imprinted. Genomic imprinting can give rise to markedly different levels of clinical penetrance depending upon the parental origin of the disease allele. Examples include SGCE mutations in myoclonus dystonia (Zimprich et al. 2001; Müller et al. 2002; Grabowski et al. 2003) and SDHD mutations in paraganglioma (Badenhop et al. 2001; Simi et al. 2005; Baysal et al. 2011). In both cases, maternal imprinting ensures that the pathologically effective mutations are almost invariably inherited from the father. Intriguingly, in one family with pseudohypoparathyroidism type $1 \mathrm{~b}$, Jan de Beur et al. (2003) reported a case of the incomplete penetrance of an imprinting mutation. These authors found that both the clinically affected and unaffected siblings had inherited the same GNAS1 allele from their affected mother, indicating that some dissociation must have occurred between the genetic GNAS1 defect responsible for the disease and its epigenetic mark. The inconsistent acquisition of a paternal epigenotype on a maternal GNAS1 allele would appear to provide evidence for the incomplete expression of a reprogramming defect that affects imprinting.

\section{Age-dependent penetrance}

Age-dependent penetrance is present if the clinical symptoms of a given disease are increasingly likely to manifest themselves with increasing age of the at-risk individual. Age-dependent penetrance has been reported for mutations in a wide variety of different human disease genes, e.g. MYBPC3 in hypertrophic cardiomyopathy (Michels et al. 2009; Page et al. 2012), LMNA in Emery-Dreifuss muscular dystrophy (Vytopil et al. 2002), MC4R in familial obesity due to melanocortin-4 receptor deficiency (Stutzmann et al. 2008), GBA in Parkinson disease (Anheim et al. 2012; Rana et al. 2013), BRCA1 and BRCA2 in breast cancer susceptibility (Chen and Parmigiani 2007; Al-Mulla et al. 2009; Mavaddat et al. 2013), MEN1 in multiple endocrine neoplasia type 1 (Machens et al. 2007), RET in multiple endocrine neoplasia type 2A (Frank-Raue et al. 2011) and $S D H D$ and $S D H B$ in predisposition to paragangliomas (Hensen et al. 2010; Hes et al. 2010). The APOE $\varepsilon 4$ allele (comprising the $\mathrm{T}$ allele of rs429358 and the $\mathrm{C}$ allele of rs7412 in cis) serves to reduce the age of onset of Alzheimer disease from 78.4 years in patients lacking the allele, to 75.3 in heterozygous carriers to 72.9 in carriers of two APOE \&4 alleles (Sando et al. 2008).

Age-dependent penetrance is particularly evident where large numbers of heterozygous carriers harbouring specific gene mutations have been identified by cascade screening, e.g. LRRK2 Gly2019Ser (rs34637584) in Parkinson disease (Latourelle et al. 2008; Healy et al. 2008; Sierra et al. 2011), GLUT1 Arg232Cys in familial idiopathic generalized epilepsy (Striano et al. 2012), RET Cys634Trp (rs77709286) in multiple endocrine neoplasia type 2A (Milos et al. 2008), ACADM Lys329Glu (rs77931234) in medium-chain acyl-CoA dehydrogenase deficiency (Andresen et al. 2012), PKP2 Gln59Leu in arrhythmogenic right ventricular cardiomyopathy (Lahtinen et al. 2008) and MYBPC3 c.2308+1G $>$ A (rs112738974) in hypertrophic cardiomyopathy (Oliva-Sandoval et al. 2010). However, there are always anomalous cases; thus, in a family segregating a pathogenic missense mutation (Arg1205His) in the vacuolar protein sorting 35 (VPS35) gene, six family members between the ages of 54 and 73 years exhibited signs of Parkinson disease, but one individual was still asymptomatic at age 86 (Nuytemans et al. 2013).

Specific mutations may sometimes differ from each other in terms of the average age of onset of clinical symptoms. Thus, for example, patients with maturity-onset diabetes of the young (MODY) who harbour mutations in exons 9 or 10 of the HNF4A gene have been found to develop disease much later (average 40 vs. 24 years) than MODY patients with mutations in exons 2-8 (Harries et al. 2008). This difference in age-related penetrance is thought to be a consequence of the exon 9 and 10 mutations being absent from three of the nine HNF4A isoforms encoded by the HNF4A gene, whereas the mutations located in exons 2-8 affect all nine isoforms.

In some cases, the clinical penetrance of a particular mutation can change quite dramatically with age. For example, the cumulative incidence among carriers of the Arg1441Gly mutation in the LRRK2 gene causing Parkinson disease was found to be $12.5 \%$ until the age of 65 years, but $83 \%$ until age 80 (Ruiz-Martínez et al. 2010). However, the penetrance of the common TTR Val30Met mutation causing autosomal dominant familial amyloid polyneuropathy has been estimated to be $1.7 \%$ until the age of 30 years, $22 \%$ until the age of 60, but still only $69 \%$ until age 90 (Hellman et al. 2008). Majounie et al. (2011) showed that the pathogenic GGGGCC hexanucleotide expansion in the $C 9$ orf 72 gene associated with a high proportion of cases of amyotrophic lateral sclerosis and frontotemporal dementia was non-penetrant in individuals younger than 35 years, $50 \%$ penetrant by age 58 but almost fully penetrant by age 80 . Age-dependent penetrance could thus provide another explanation for why some putatively pathological mutations listed in HGMD 
are present in apparently healthy individuals from the 1000 Genomes Project.

A glimpse of the way ahead is provided by a recent study of symptomatic and asymptomatic carriers of a specific granulin $(G R N)$ mutation (Thr272Ser) responsible for autosomal dominant frontotemporal lobar degeneration (FTLD), a disease whose onset typically occurs in the sixth decade of life (Milanesi et al. 2013). Unsurprisingly, both the symptomatic and asymptomatic $G R N$ mutation carriers had lower serum levels of progranulin than non-carriers. However, using whole-genome microarray analysis, the leukocyte expression of the TMEM40 and LY6G6F genes was found to be significantly higher in FTLD patients harbouring GRN mutations as compared to asymptomatic carriers. Further, elevated expression of the genes was correlated with increased brain damage and could therefore be directly related to the pathology of the disease (Milanesi et al. 2013).

\section{Epigenetic influences on disease penetrance}

As briefly discussed above in the context of the influence of gender upon penetrance, epigenetic modifications may also account for incomplete penetrance. Thus, when monozygotic twins are discordant for disease phenotypes, epigenetic differences should be considered (Wong et al. 2005; Kaminsky et al. 2009; Gordon et al. 2011; 2012). Indeed, monozygotic twins have been reported who differ both in relation to a specific clinical phenotype and in terms of an epigenotype. For example, monozygotic twins discordant for childhood leukaemia have been found to have discordant BRCAl methylation status (Galetzka et al. 2012). Similarly, hypermethylation of SLC6A4, encoding the serotonin transporter, has been reported in one member of a monozygotic twin pair discordant for bipolar disorder (Sugawara et al. 2011).

Epigenetic differences may also contribute to incomplete penetrance in other conditions such as asthma, where DNA methylation has been reported to modulate the risk of disease conferred by genetic variants at the zona pellucida binding protein 2 (ZPBP2; Berlivet et al. 2012), forkhead box P3 (FOXP3; Runyon et al. 2012), interferon- $\gamma$ (IFNG; Runyon et al. 2012) and interleukin-4 receptor (ILAR; SotoRamírez et al. 2013) gene loci.

A special case of imprinting is provided by $\mathrm{X}$-inactivation (Dobyns et al. 2004). When a disease gene is $\mathrm{X}$-linked, skewed $\mathrm{X}$-inactivation can cause variable penetrance of pathogenic mutations in female carriers (Van den Veyver 2001). Examples of this phenomenon involve mutations in the TIMM8A gene (Xq22.1) in dystoniadeafness syndrome (Plenge et al. 1999), the EBP gene (Xp11.23) in X-linked dominant chondrodysplasia punctata (Shirahama et al. 2003), the FLNA gene (Xq28) in otopalatodigital type 1 syndrome (Hidalgo-Bravo et al. 2005), the $A B C D 1$ gene (Xq28) in a family with $\mathrm{X}$-linked adrenoleukodystrophy (Wang et al. 2013b) and the ZIC3 gene $(\mathrm{Xq} 26.3)$ in a family with complex heart defects (Chhin et al. 2007). It should, however, be pointed out that some ZIC3 mutations are characterized by reduced penetrance in males, a finding that cannot be explained by skewed X-inactivation (Mégarbané et al. 2000).

\section{Gene-environment interactions and penetrance}

The environment, in its broadest sense, will often influence clinical penetrance, either ameliorating or exacerbating the impact of heritable genetic variants (Hunter 2005). Indeed, environmental modifiers of disease penetrance (e.g. diet, alcohol intake, drugs, metabolic syndrome) have long been known to influence the penetrance of HFE C282Y homozygosity in haemochromatosis (Beutler 2003; Rossi et al. 2008; Deugnier and Mosser 2008).

One way to explore the relative contribution of genes and environment is by studying monozygotic twins harbouring the same pathogenic mutation(s) and sharing the same genetic background. Although the vast majority of such monozygotic twin pairs have been found to be concordant in terms of their clinical phenotypes (e.g. Miesfeldt et al. 1998; Munhoz et al. 2008; McDade et al. 2012), others are quite discordant (Matsuo et al. 2000; Amann et al. 2001; Martin et al. 2003; Holmgren et al. 2004; Lachmann et al. 2004; Czlonkowska et al. 2009; Biegstraaten et al. 2011; Fencl et al. 2012; Iatropoulos et al. 2012), suggesting that the environment can often play an important role in determining both the penetrance and expressivity of pathological mutations. [It should be borne in mind that there are various alternative genetic explanations for discordant phenotypes in monozygotic twins, including de novo post-zygotic mutation (Kentsis et al. 2009; Vogt et al. 2011), compensatory mutations (Mankad et al. 2006) and somatic copy number variation (Bruder et al. 2008) that obviate the need for a major contribution from the environment, as well as acquired epigenetic differences (Galetzka et al. 2012; Bennett et al. 2008)].

An environmental influence on penetrance is perhaps at its most evident in cancer susceptibility (Houlston and Peto 2004; Shen 2009). Indeed, an environmental component is very important in colorectal cancer where inherited genetic variants at a number of different loci interact primarily with dietary variables and overweight to confer risk (Hutter et al. 2012; Siegert et al. 2013). In similar vein, inherited differences in skin pigmentation influence the risk of melanoma, but this risk is further modified both by latitude of habitation and lifestyle choices (van der Velden et al. 2001; Bishop et al. 2002; Begg et al. 2005; Meyle and 
Guldberg 2009; Scherer and Kumar 2010). Parity and breast feeding are both known to be modifiers of risk of breast/ovarian cancer in BRCAl mutation carriers (McLaughlin et al. 2007; Jernström et al. 2004; Cullinane et al. 2005; Antoniou et al. 2006). Another example of a gene-environment interaction in the context of cancer is provided by cytochrome $\mathrm{P} 450$ gene variants that may influence cancer risk by virtue of their roles in xenobiotic metabolism, detoxification of carcinogens, and to a lesser extent the bioactivation of procarcinogens (RodriguezAntona et al. 2010). Lung cancer provides an excellent example of the interaction of genes and environment. Three different GWAS, published virtually simultaneously, provided the first convincing evidence for an association between heritable genetic variation at the nicotinic acetylcholine receptor CHRNA5/CHRNA3/CHRNB4 locus on chromosome 15q25.1 and lung cancer (Amos et al. 2008; Hung et al. 2008; Thorgeirsson et al. 2008). Although allele T of SNP rs1051730, a synonymous variant located within exon 5 of the CHRNA3 gene, was found to be strongly associated with smoking quantity, the issue of whether the association with lung cancer was direct or indirect (i.e. mediated through cigarette smoking and nicotine dependence) remained unclear. Galvan and Dragan (2009) performed a meta-analysis of reported studies of the $15 \mathrm{q} 25$ region and found that this locus was not associated with lung cancer risk in never-smokers. This lack of effect argued for an indirect effect of genetic variation at the $15 \mathrm{q} 25$ locus on lung cancer risk via an association between these variants and smoking/nicotine dependence. However, Wang et al. (2010b) subsequently examined the relationship between rs1051730 and lung cancer and concluded that, in addition to its indirect influence on disease risk (through smoking behaviour), this variant also exerted a rather larger (and direct) effect. Kaur-Knudsen et al. (2011) concurred, demonstrating that homozygosity for rs 1051730 was associated with a smoking behaviour-adjusted relative risk of lung cancer of 1.6, indicating that rs 1051730 is associated with an additional risk of lung cancer over and above that derived from its effect on smoking behaviour. Finally, in a lung cancer case-control study, VanderWeele et al. (2012) employed two 15q25.1 SNPs, rs8034191 and rs1051730, to show that the proportion of increased risk due to smoking was only $3.2 \%$ and that the association of the $15 \mathrm{q} 25$ variants with lung cancer operates primarily through pathways other than smoking behaviour. All of the above notwithstanding, the risk of lung cancer conferred directly or indirectly by genetic variants on $15 q 25$ would be small if the individual concerned simply opted not to smoke (Brennan et al. 2011).

The penetrance of genetic variants conferring susceptibility to infectious disease is clearly contingent upon exposure to the specific pathogens concerned (Vannberg et al. 2011; Chapman and Hill 2012). One example is the
CCR5 32-bp (c.554del32) deletion which is associated with a lower rate of HIV infection and a delay in the onset of AIDS (Smith et al. 1997). Sex may also play a role in some conditions; thus, in multiple sclerosis, women appear to be more responsive to the environmental risk factors that cause the disease (Goodin 2012; O'Gorman et al. 2012).

Diet is also an important modifier of clinical penetrance. Thus, an inherited predisposition to obesity (exemplified by the association between dietary fat intake and obesity in carriers of the PPARG2 Pro12Ala allele; Memisoglu et al. 2003) is in principle modifiable by diet (Walters et al. 2010; Ramachandrappa and Farooqi 2011). Similarly, the impact of genetic variants at the FTO locus on risk of obesity can be attenuated by physical activity (Kilpeläinen et al. 2011). Diet is also an important modifier of clinical penetrance in phenylketonuria, as mentioned in the "Introduction" to this review, where the penetrance of the condition can be very substantially reduced by restricting dietary phenylalanine (van Spronsen 2010).

Heavy coffee drinkers have been known for some time to have a reduced risk of developing Parkinson disease. However, the risk of developing Parkinson disease has been found to be reduced even further for heavy coffee drinkers by a specific variant in the GRIN2A gene; compared to light coffee drinkers with an rs4998386_CC genotype, heavy coffee drinkers with the same genotype have an $18 \%$ lower risk, whereas heavy coffee drinkers with an rs4998386_TC genotype have a $59 \%$ lower risk (Hamza et al. 2011).

More unusually, altitude has been reported to act as a modifier of the phenotypic severity of hereditary paraganglioma type 1 caused by mutations in the succinate dehydrogenase D (SDHD) gene (Astrom et al. 2003). Since chronic hypoxic stimulation at high altitude causes sporadic carotid body paragangliomas, Astrom et al. (2003) proposed that SDHD might be involved in oxygen sensing. Thus, whilst $S D H D$ mutations could impair oxygen sensing, low altitude may serve to reduce the penetrance of these mutations.

The clinical penetrance of psychological disorders and traits has long been known to be strongly influenced by gene-environment interactions (Dick 2011). For example, a 44-bp deletion/insertion polymorphism in the promoter region of the serotonin transporter gene SLC6A4 was reported to be associated with depression after stressful life events (Caspi et al. 2003). Recently, Klengel et al. (2013) gave us a glimpse of the likely complexity of the mechanisms underlying gene-environment interactions in the context of psychological disorders. These authors demonstrated that a risk variant for post-traumatic stress disorder and major depression in the FK506-binding protein 5 gene $(F K B P 5)$ is demethylated in several cell types in children exposed to trauma. This demethylation persists into 
adulthood and confers an increased risk of developing disease. In FKBP5 risk allele carriers, excessive cortisol release during early life stress leads to demethylation within the glucocorticoid-responsive elements of FKBP5 in intron 7 with the consequence of long-lasting disruption of the ultra-short feedback loop that balances FKBP5 and glucocorticoid receptor activity, causing dysregulation of the stress hormone system. The FKBP5 risk allele corresponds to a functional polymorphism located in intron 2 of the FKBP5 gene that alters the chromatin interaction between the transcriptional start site and long-range enhancers, thereby increasing the transcriptional activity of FKBP5 over and above that of the wild-type allele. Only the risk allele is able to form a three-dimensional complex which includes RNA polymerase II and a glucocorticoidresponsive element located within intron 7 of FKBP5. Enhanced transcription of the risk allele facilitates the PolII-dependent demethylation in intron 7 in response to elevated glucocorticoids under early life stressful conditions. The reduced methylation of intron $7 \mathrm{CpGs}$ leads to increased induction of FKBP5 by glucocorticoid receptor activation, especially in risk allele carriers, representing an enhancement of the ultra-short feedback loop leading to increased glucocorticoid receptor resistance. If this occurs during developmentally critical periods, then the methylation patterns will remain stable over time (Klengel et al. 2013). Hence, the demethylation in FKBP5 intron 7 depends upon both childhood trauma and the sequence variant in intron 2 , in a tripartite gene-environment interaction (Szyf 2012).

The above examples represent the tip of the iceberg because the clinical penetrance of most monogenic conditions and all complex disease is likely to be influenced by the environment in some way or another. A further glimpse of this complexity is perhaps provided by Kallberg et al. (2007) who reported interactions between HLA-DRB1 SE alleles, the A allele of the PTPN22 Arg620Trp (rs2476601) polymorphism and smoking in conferring risk of rheumatoid arthritis. Gene-environment interactions are also evident in asthma (Custovic et al. 2012; Chang et al. 2012) and eczema (Bisgaard et al. 2008) and will become increasingly apparent in other disorders as new techniques are developed to identify them (Aschard et al. 2012). To this end, mouse models are beginning to come into their own as a means to study the role of gene-environment interactions in the aetiology of human disorders; by these means, short-term gestational hypoxia has been found to increase the penetrance of vertebral defects in congenital scoliosis (Sparrow et al. 2012).

\section{Conclusions}

A holy grail of human medical genetics is to be able to deduce the likely clinical phenotype of an individual from their genotype or genomic sequence. It was once perhaps naively assumed that, at least for "monogenic" disorders, genotype-phenotype relationships would be that simple, and also fairly straightforward to discern. However, it has been clear for some time that it is inappropriate to regard such disorders as either simple or monogenic in any strict sense. Further, in many cases, the reality is that we cannot readily draw straight lines of causation from known genotypes to specific clinical phenotypes. This is because instances abound of individuals who harbour a diseaseassociated mutation/genotype, but who do not express certain features of the disease or who may even be asymptomatic. This phenomenon of reduced penetrance may or may not be the norm, but it is far from being a rare exception. Our appreciation of its full extent is still emerging, although some of the different mechanisms underlying reduced penetrance are now becoming apparent (Fig. 1).

It has become clear from large-scale sequencing studies that many individuals in the general population harbour
Fig. 1 Some of the different mechanisms underlying the phenomenon of reduced penetrance in human inherited disease

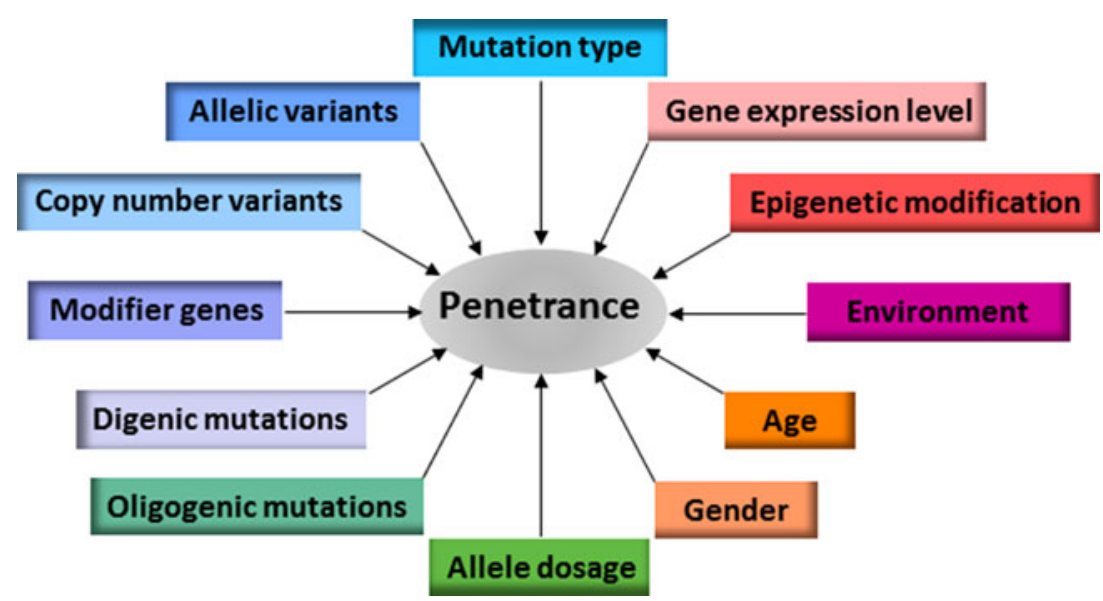


large numbers of potentially disadvantageous variants without suffering any obvious ill effects (The 1000 Genomes Project Consortium 2010; MacArthur et al. 2012; Xue et al. 2012; Shen et al. 2013a). Thus, it would appear that many mutations are, on their own, insufficient to cause disease and need to occur in the presence of other genetic variants, either allelic or non-allelic, as well as facultative environmental factor(s), for a disease state to ensue. Indeed, many pathological mutations may only be conditionally pathogenic, exerting a detrimental effect only if and when the genetic and external environments interact to push the phenotype over some notional threshold into pathology.

Penetrance is best thought of as being a genotype-specific rather than a gene-specific or disease-specific phenomenon. Thus, in any given disease gene, some mutations may exhibit complete penetrance, whereas others may show incomplete or even quite low penetrance. Generally speaking, mutations that display low penetrance also tend to exert milder effects on the clinical phenotype and/or protein function, while the more highly penetrant a mutation is, the less frequent it is likely to be in the general population (Coventry et al. 2010; Marth et al. 2011; Gorlov et al. 2011; Tennessen et al. 2012; Nelson et al. 2012; Subramanian 2012; Fu et al. 2013). Whereas highly penetrant mutations may exert their pathogenic effects with relatively little interaction with other genetic or environmental factors, low-penetrance mutations are generally characterized by significant gene-gene and gene-environment interactions (Cordell 2009). Different combinations of such variants may contribute to the variable penetrance characteristic of both monogenic and complex disease.

Whatever the molecular basis may be in the case of a given mutation, reduced penetrance is in general likely to present a serious impediment to the implementation of any scheme designed to classify the pathological significance of human genetic variants (e.g. Plon et al. 2008; Tavtigian et al. 2008). Reduced penetrance is also likely to present problems in identifying pathological mutations in wholegenome/exome sequencing programmes unless it is explicitly built into the disease models being considered (Varga et al. 2013). Despite the complexities it introduces, it also offers hope in the sense that if we are able to identify environmental factors, drugs or other types of intervention that serve to reduce the penetrance of a given pathological variant, or alternatively delay the onset of its pathological sequelae beyond the natural lifespan of its carrier, we shall have a whole new range of therapeutic approaches at our disposal.

Human genetic variation occurs as a continuum ranging from neutral polymorphisms, through functional polymorphisms and disease susceptibility variants to true pathological mutations with high penetrance. However, in addition and as discussed above, it has become increasingly clear that our genomes contain many 'putatively disadvantageous variants' that are probably insufficient on their own to cause disease, but nevertheless still have the potential to contribute to pathogenesis. Since it has also become clear that many genetic disorders are not monogenic as originally supposed, but may instead involve mutations in two or more genes, we speculate that different combinations of pathological mutations with low penetrance, functional polymorphisms, disease susceptibility variants and 'putatively disadvantageous variants' may vary quite considerably in terms of their net functional and hence clinical effect. Such combinations are likely to exert an influence on the age of onset and/or clinical severity of the disease in question.

The rationale of genetic studies of complex phenotypes has generally relied upon either the 'common disease, common variant (CDCV)' hypothesis or the 'common disease, rare variant (CDRV)' hypothesis. The former postulates that complex phenotypes result from the cumulative effects of a number of common variants, each with a modest effect size and relatively low penetrance. The latter proposes that complex phenotypes result from multiple rare variants, each with relatively high penetrance and large effect sizes (Schork et al. 2009). It is highly likely that both rare and common alleles will contribute to complex phenotypes and so effect sizes may be expected to differ quite widely, with rare variants with large effects complemented by a large number of frequent variants with small effects. The clinical phenotypes of complex phenotypes are therefore likely to be due to individual effects of, and interaction between, multiple causative or contributory alleles, as well as non-genetic determinants.

The full relevance of digenic and oligogenic inheritance to the phenomenon of incomplete or variable penetrance remains to be elucidated. If, however, it turns out that digenic and oligogenic conditions are more common than originally anticipated, then many disease contributory variants will have evaded purifying selection, and hence those variants that in combination (but not individually) have significant pathogenic potential will not be as infrequent as might be expected under the CDRV hypothesis. Since both multiple common and rare variants may be involved in conferring disease susceptibility, we are not obliged to favour either the CDCV hypothesis or the CDRV hypothesis. Moreover, in view of the likely complexity of the gene-gene interactions involved, we concur with Lupski et al. (2011) that "for a given individual, what is important to know is not only the number and location of pathogenic variants taken one at a time, but also the unique composition of his or her genome-wide mutational burden".

Lupski et al. (2011) charted progress on the road to a unified genetic model for human disease and opined that such a model should unite categories of diseases, previously held to be distinct entities, as part of a continuum 
which would include chromosomal syndromes, genomic disorders, Mendelian traits and common diseases or complex traits. Concurring with this view, we envisage an integrated concept of genetic aetiology in which different types of mutation (from single base substitutions to copy number variants), different combinations of mutations in multiple genes (whether in homozygosity or heterozygosity), cis-acting or trans-acting modifiers, common variants, rare variants, de novo variants and even somatic variants, jointly serve to exacerbate or ameliorate a given clinical phenotype. Further, to explain the scale of reduced penetrance, we need to conceptualize clinical phenotypes as being derived, potentially at least, from the expression of different genetic variants in two or more genes. On the basis of the data collated for this review, it seems reasonable to conclude that digenic, oligogenic and polygenic influences are much more frequent than has perhaps hitherto been realized. Unravelling such influences will undoubtedly be key to understanding the molecular basis of reduced penetrance. The impact of disease genotypes may also be modified by epigenetic and environmental factors, allowing both for synergistic and antagonistic interactions resulting in highly individualized contributions to the phenotype (whether deleterious or protective) that will variously perturb the balance of specific biological pathways so as to give rise to disease.

With the advent of next-generation sequencing, very large numbers of genetic variants are being detected in individual genomes and it has been necessary to develop new algorithms to identify those variants which are of key functional/clinical importance. However, if in using these tools, we focus exclusively on single infrequent variants under the assumption that they will invariably exert their effects in splendid isolation, then there is a very real danger that we shall inadvertently exclude from consideration those more frequent variants with modest effects, blithely ignoring their potential for interaction with the rare variants. The irony would then be that, despite having the requisite mutation and polymorphism data available, the molecular basis of genotype-phenotype relationships in many inherited diseases (including, of course, the phenomenon of reduced penetrance) could still remain unintelligible. The alternative, anticipating multigenic influences on the clinical phenotypes associated with disorders traditionally regarded as being monogenic, should not only to lead to new insights into the nature of reduced penetrance, but is also likely to improve our understanding of the nature of complex disease.

Acknowledgments The authors are grateful to Peter Stenson for provision of HGMD mutation data. DNC acknowledges receipt of financial support from BIOBASE GmbH through a licence agreement with Cardiff University. CTS is supported by the Wellcome Trust (098051).
Open Access This article is distributed under the terms of the Creative Commons Attribution License which permits any use, distribution, and reproduction in any medium, provided the original author(s) and the source are credited.

\section{References}

Abifadel M, Rabès JP, Jambart S, Halaby G, Gannagé-Yared MH, Sarkis A, Beaino G, Varret M, Salem N, Corbani S, Aydénian H, Junien C, Munnich A, Boileau C (2009) The molecular basis of familial hypercholesterolemia in Lebanon: spectrum of $L D L R$ mutations and role of PCSK9 as a modifier gene. Hum Mutat 30:E682-E691

Abu-Safieh L, Al-Anazi S, Al-Abdi L, Hashem M, Alkuraya H, Alamr M, Sirelkhatim MO, Al-Hassnan Z, Alkuraya B, Mohamed JY, Al-Salem A, Alrashed M, Faqeih E, Softah A, Al-Hashem A, Wali S, Rahbeeni Z, Alsayed M, Khan AO, AlGazali L, Taschner PE, Al-Hazzaa S, Alkuraya FS (2012) In search of triallelism in Bardet-Biedl syndrome. Eur $\mathrm{J}$ Hum Genet 20:420-427

Ackermann B, Kröber S, Torres-Benito L, Borgmann A, Peters M, Hosseini Barkooie SM, Tejero R, Jakubik M, Schreml J, Milbradt J, Wunderlich TF, Riessland M, Tabares L, Wirth B (2013) Plastin 3 ameliorates spinal muscular atrophy via delayed axon pruning and improves neuromuscular junction functionality. Hum Mol Genet 22:1328-1347

Addis M, Meloni C, Tosetto E, Ceol M, Cristofaro R, Melis MA, Vercelloni P, Del Prete D, Marra G, Anglani F (2013) An atypical Dent's disease phenotype caused by co-inheritance of mutations at CLCN5 and OCRL genes. Eur J Hum Genet 21:687-690

Aganna E, Aksentijevich I, Hitman GA, Kastner DL, Hoepelman AI, Posma FD, Zweers EJ, McDermott MF (2001) Tumor necrosis factor receptor-associated periodic syndrome (TRAPS) in a Dutch family: evidence for a TNFRSF1A mutation with reduced penetrance. Eur J Hum Genet 9:63-66

Ahluwalia JK, Hariharan M, Bargaje R, Pillai B, Brahmachari V (2009) Incomplete penetrance and variable expressivity: is there a microRNA connection? BioEssays 31:981-992

Akerman BR, Lemass H, Chow LM, Lambert DM, Greenberg C, Bibeau C, Mamer OA, Treacy EP (1999) Trimethylaminuria is caused by mutations of the FMO3 gene in a North American cohort. Mol Genet Metab 68:24-31

Aksentijevich I, Galon J, Soares M, Mansfield E, Hull K, Oh HH, Goldbach-Mansky R, Dean J, Athreya B, Reginato AJ, Henrickson M, Pons-Estel B, O'Shea JJ, Kastner DL (2001) The tumor-necrosis-factor receptor-associated periodic syndrome: new mutations in TNFRSF1A, ancestral origins, genotypephenotype studies, and evidence for further genetic heterogeneity of periodic fevers. Am J Hum Genet 69:301-314

Alberobello AT, Congedo V, Liu H, Cochran C, Skarulis MC, Forrest D, Celi FS (2011) An intronic SNP in the thyroid hormone receptor $\beta$ gene is associated with pituitary cell-specific overexpression of a mutant thyroid hormone receptor $\beta 2(\mathrm{R} 338 \mathrm{~W})$ in the index case of pituitary-selective resistance to thyroid hormone. J. Transl. Med. 9:144

Albers CA, Paul DS, Schulze H, Freson K, Stephens JC, Smethurst PA, Jolley JD, Cvejic A, Kostadima M, Bertone P, Breuning MH, Debili N, Deloukas P, Favier R, Fiedler J, Hobbs CM, Huang N, Hurles ME, Kiddle G, Krapels I, Nurden P, Ruivenkamp CA, Sambrook JG, Smith K, Stemple DL, Strauss G, Thys C, van Geet C, Newbury-Ecob R, Ouwehand WH, Ghevaert C (2012) Compound inheritance of a low-frequency 
regulatory $\mathrm{SNP}$ and a rare null mutation in exon-junction complex subunit RBM8A causes TAR syndrome. Nat Genet 44:435-439

Al-Mulla F, Bland JM, Serratt D, Miller J, Chu C, Taylor GT (2009) Age-dependent penetrance of different germline mutations in the BRCA1 gene. J Clin Pathol 62:350-356

Alonso I, Jardim LB, Artigalas O, Saraiva-Pereira ML, Matsuura T, Ashizawa T, Sequeiros J, Silveira I (2006) Reduced penetrance of intermediate size alleles in spinocerebellar ataxia type 10 . Neurology 66:1602-1604

Altès A, Bach V, Ruiz A, Esteve A, Felez J, Remacha AF, Sardà MP, Baiget M (2009) Mutations in HAMP and HJV genes and their impact on expression of clinical hemochromatosis in a cohort of 100 Spanish patients homozygous for the C282Y mutation of HFE gene. Ann Hematol 88:951-955

Alves MM, Sribudiani Y, Brouwer RW, Amiel J, Antiñolo G, Borrego S, Ceccherini I, Chakravarti A, Fernández RM, GarciaBarcelo MM, Griseri P, Lyonnet S, Tam PK, van Ijcken WF, Eggen BJ, Te Meerman GJ, Hofstra RM (2013) Contribution of rare and common variants determine complex diseases-Hirschsprung disease as a model. Dev Biol (in press). doi:10.1016/ j.ydbio.2013.05.019

Amann ST, Gates LK, Aston CE, Pandya A, Whitcomb DC (2001) Expression and penetrance of the hereditary pancreatitis phenotype in monozygotic twins. Gut 48:542-547

Amin AS, Giudicessi JR, Tijsen AJ, Spanjaart AM, Reckman YJ, Klemens CA, Tanck MW, Kapplinger JD, Hofman N, Sinner MF, Müller M, Wijnen WJ, Tan HL, Bezzina CR, Creemers EE, Wilde AA, Ackerman MJ, Pinto YM (2012) Variants in the $3^{\prime}$ untranslated region of the KCNQ1-encoded Kv7.1 potassium channel modify disease severity in patients with type 1 long QT syndrome in an allele-specific manner. Eur Heart J 33:714-723

Ammerlaan AC, Ararou A, Houben MP, Baas F, Tijssen CC, Teepen JL, Wesseling P, Hulsebos TJ (2008) Long-term survival and transmission of INII-mutation via nonpenetrant males in a family with rhabdoid tumour predisposition syndrome. $\mathrm{Br} \mathrm{J}$ Cancer 98:474-479

Amos CI, Wu X, Broderick P, Gorlov IP, Gu J, Eisen T, Dong Q, Zhang Q, Gu X, Vijayakrishnan J, Sullivan K, Matakidou A, Wang Y, Mills G, Doheny K, Tsai YY, Chen WV, Shete S, Spitz MR, Houlston RS (2008) Genome-wide association scan of tag SNPs identifies a susceptibility locus for lung cancer at $15 \mathrm{q} 25.1$. Nat Genet 40:616-622

Andreasen C, Nielsen JB, Refsgaard L, Holst AG, Christensen AH, Andreasen L, Sajadieh A, Haunsø S, Svendsen JH, Olesen MS (2013) New population-based exome data are questioning the pathogenicity of previously cardiomyopathy-associated genetic variants. Eur J Hum Genet (in press)

Andresen BS, Lund AM, Hougaard DM, Christensen E, Gahrn B, Christensen M, Bross P, Vested A, Simonsen H, Skogstrand K, Olpin S, Brandt NJ, Skovby F, Nørgaard-Pedersen B, Gregersen N (2012) MCAD deficiency in Denmark. Mol Genet Metab 106:175-188

Anheim M, Elbaz A, Lesage S, Durr A, Condroyer C, Viallet F, Pollak $\mathrm{P}$, Bonaïti B, Bonaïti-Pellié C, Brice A, French Parkinson Disease Genetic Group (2012) Penetrance of Parkinson disease in glucocerebrosidase gene mutation carriers. Neurology 78:417-420

Antoniou AC, Sinilnikova OM, Simard J, Léoné M, Dumont M, Neuhausen SL, Struewing JP, Stoppa-Lyonnet D, Barjhoux L, Hughes DJ, Coupier I, Belotti M, Lasset C, Bonadona V, Bignon YJ; Genetic Modifiers of Cancer Risk in BRCA1/2 Mutation Carriers Study (GEMO), Rebbeck TR, Wagner T, Lynch HT, Domchek SM, Nathanson KL, Garber JE, Weitzel J, Narod SA, Tomlinson G, Olopade OI, Godwin A, Isaacs C, Jakubowska A, Lubinski J, Gronwald J, Górski B, Byrski T, Huzarski T, Peock S, Cook M, Baynes C, Murray A, Rogers M, Daly PA, Dorkins
$\mathrm{H}$; Epidemiological Study of BRCA1 and BRCA2 Mutation Carriers (EMBRACE), Schmutzler RK, Versmold B, Engel C, Meindl A, Arnold N, Niederacher D, Deissler H; German Consortium for Hereditary Breast and Ovarian Cancer (GCHBOC), Spurdle AB, Chen X, Waddell N, Cloonan N; Kathleen Cuningham Consortium for Research into Familial Breast Cancer (kConFab), Kirchhoff T, Offit K, Friedman E, Kaufmann B, Laitman Y, Galore G, Rennert G, Lejbkowicz F, Raskin L, Andrulis IL, Ilyushik E, Ozcelik H, Devilee P, Vreeswijk MP, Greene MH, Prindiville SA, Osorio A, Benitez J, Zikan M, Szabo CI, Kilpivaara O, Nevanlinna H, Hamann U, Durocher F, Arason A, Couch FJ, Easton DF, Chenevix-Trench $\mathrm{G}$; Consortium of Investigators of Modifiers of BRCA1/2 (CIMBA) (2007) RAD51 135G $>$ C modifies breast cancer risk among BRCA2 mutation carriers: results from a combined analysis of 19 studies. Am J Hum Genet 81:1186-1200

Antoniou AC, Chenevix-Trench G (2010) Common genetic variants and cancer risk in Mendelian cancer syndromes. Curr Opin Genet Dev 20:299-307

Antoniou AC, Shenton A, Maher ER, Watson E, Woodward E, Lalloo F, Easton DF, Evans DG (2006) Parity and breast cancer risk among $B R C A 1$ and BRCA2 mutation carriers. Breast Cancer Res 8:R72

Apetri AC, Vanik DL, Surewicz WK (2005) Polymorphism at residue 129 modulates the conformational conversion of the D178N variant of human prion protein 90-231. Biochemistry 44:15880-15888

Arbini AA, Bodkin D, Lopaciuk S, Bauer KA (1994) Molecular analysis of Polish patients with factor VII deficiency. Blood 84:2214-2220

Arnold WV, Fertala A (2013) Skeletal diseases caused by mutations that affect collagen structure and function. Int $\mathrm{J}$ Biochem Cell Biol 45:1556-1567

Arnold M, Ellwanger DC, Hartsperger ML, Pfeufer A, Stümpflen V (2012) Cis-acting polymorphisms affect complex traits through modifications of microRNA regulation pathways. PLoS ONE 7:e36694

Arzel-Hézode M, Sternberg D, Tabti N, Vicart S, Goizet C, Eymard B, Fontaine B, Fournier E (2010) Homozygosity for dominant mutations increases severity of muscle channelopathies. Muscle Nerve 41:470-477

Asan, Xu Y, Jiang H, Tyler-Smith C, Xue Y, Jiang T, Wang J, Wu M, Liu X, Tian G, Wang J, Wang J, Yang H, Zhang X (2011) Comprehensive comparison of three commercial human wholeexome capture platforms. Genome Biol 12:R95

Aschard H, Lutz S, Maus B, Duell EJ, Fingerlin TE, Chatterjee N, Kraft P, Van Steen K (2012) Challenges and opportunities in genome-wide environmental interaction (GWEI) studies. Hum Genet 131:1591-1613

Ashley EA, Butte AJ, Wheeler MT, Chen R, Klein TE, Dewey FE, Dudley JT, Ormond KE, Pavlovic A, Morgan AA, Pushkarev D, Neff NF, Hudgins L, Gong L, Hodges LM, Berlin DS, Thorn CF, Sangkuhl K, Hebert JM, Woon M, Sagreiya H, Whaley R, Knowles JW, Chou MF, Thakuria JV, Rosenbaum AM, Zaranek AW, Church GM, Greely HT, Quake SR, Altman RB (2010) Clinical assessment incorporating a personal genome. Lancet 375:1525-1535

Astrom K, Cohen JE, Willett-Brozick JE, Aston CE, Baysal BE (2003) Altitude is a phenotypic modifier in hereditary paraganglioma type 1: evidence for an oxygen-sensing defect. Hum Genet 113:228-237

Austin ED, Phillips JA, Cogan JD, Hamid R, Yu C, Stanton KC, Phillips CA, Wheeler LA, Robbins IM, Newman JH, Loyd JE (2009a) Truncating and missense BMPR2 mutations differentially affect the severity of heritable pulmonary arterial hypertension. Respir Res 10:87 
Austin ED, Cogan JD, West JD, Hedges LK, Hamid R, Dawson EP, Wheeler LA, Parl FF, Loyd JE, Phillips JA 3rd (2009b) Alterations in oestrogen metabolism: implications for higher penetrance of familial pulmonary arterial hypertension in females. Eur Respir J 34:1093-1009

Azaiez H, Chamberlin GP, Fischer SM, Welp CL, Prasad SD, Taggart RT, del Castillo I, Van Camp G, Smith RJ (2004) GJB2: the spectrum of deafness-causing allele variants and their phenotype. Hum Mutat 24:305-311

Azmanov DN, Dimitrova S, Florez L, Cherninkova S, Draganov D, Morar B, Saat R, Juan M, Arostegui JI, Ganguly S, Soodyall H, Chakrabarti S, Padh H, López-Nevot MA, Chernodrinska V, Anguelov B, Majumder P, Angelova L, Kaneva R, Mackey DA, Tournev I, Kalaydjieva L (2011) LTBP2 and CYP1B1 mutations and associated ocular phenotypes in the Roma/Gypsy founder population. Eur J Hum Genet 19:326-333

Badano JL, Katsanis N (2003) Beyond Mendel: an evolving view of human genetic disease transmission. Nat Rev Genet 3:779-789

Badano JL, Kim JC, Hoskins BE, Lewis RA, Ansley SJ, Cutler DJ, Castellan C, Beales PL, Leroux MR, Katsanis N (2003) Heterozygous mutations in $B B S 1, B B S 2$ and $B B S 6$ have a potential epistatic effect on Bardet-Biedl patients with two mutations at a second BBS locus. Hum Mol Genet 12:1651-1659

Badano JL, Leitch CC, Ansley SJ, May-Simera H, Lawson S, Lewis RA, Beales PL, Dietz HC, Fisher S, Katsanis N (2006) Dissection of epistasis in oligogenic Bardet-Biedl syndrome. Nature 439:326-330

Badenhop RF, Cherian S, Lord RS, Baysal BE, Taschner PE, Schofield PR (2001) Novel mutations in the SDHD gene in pedigrees with familial carotid body paraganglioma and sensorineural hearing loss. Genes Chromosome Cancer 31:255-263

Balwani M, Fuerstman L, Kornreich R, Edelmann L, Desnick RJ (2010) Type 1 Gaucher disease: significant disease manifestations in "asymptomatic" homozygotes. Arch Intern Med 170:1463-1469

Baradaran-Heravi A, Cho KS, Tolhuis B, Sanyal M, Morozova O, Morimoto M, Elizondo LI, Bridgewater D, Lubieniecka J, Beirnes K, Myung C, Leung D, Fam HK, Choi K, Huang Y, Dionis KY, Zonana J, Keller K, Stenzel P, Mayfield C, Lücke T, Bokenkamp A, Marra MA, van Lohuizen M, Lewis DB, Shaw C, Boerkoel CF (2012) Penetrance of biallelic SMARCAL1 mutations is associated with environmental and genetic disturbances of gene expression. Hum Mol Genet 21:2572-2587

Basel-Vanagaite L, Pelet A, Steiner Z, Munnich A, Rozenbach Y, Shohat M, Lyonnet S (2007) Allele dosage-dependent penetrance of RET proto-oncogene in an Israeli-Arab inbred family segregating Hirschsprung disease. Eur J Hum Genet 15:242-245

Baysal BE, McKay SE, Kim YJ, Zhang Z, Alila L, Willett-Brozick JE, Pacak K, Kim TH, Shadel GS (2011) Genomic imprinting at a boundary element flanking the $S D H D$ locus. Hum Mol Genet 20:4452-4461

Beales PL, Badano JL, Ross AJ, Ansley SJ, Hoskins BE, Kirsten B, Mein CA, Froguel P, Scambler PJ, Lewis RA, Lupski JR, Katsanis N (2003) Genetic interaction of BBS1 mutations with alleles at other BBS loci can result in non-Mendelian BardetBiedl syndrome. Am J Hum Genet 72:1187-1199

Beckmann JS, Estivill X, Antonarakis SE (2007) Copy number variants and genetic traits: closer to the resolution of phenotypic to genotypic variability. Nat Rev Genet 8:639-646

Begg CB, Orlow I, Hummer AJ, Armstrong BK, Kricker A, Marrett LD, Millikan RC, Gruber SB, Anton-Culver H, Zanetti R, Gallagher RP, Dwyer T, Rebbeck TR, Mitra N, Busam K, From L, Berwick M, Genes Environment and Melanoma Study Group (2005) Lifetime risk of melanoma in $C D K N 2 A$ mutation carriers in a population-based sample. J Natl Cancer Inst 97:1507-1515

Bell CJ, Dinwiddie DL, Miller NA, Hateley SL, Ganusova EE, Mudge J, Langley RJ, Zhang L, Lee CC, Schilkey FD, Sheth V,
Woodward JE, Peckham HE, Schroth GP, Kim RW, Kingsmore SF (2011) Carrier testing for severe childhood recessive diseases by next-generation sequencing. Sci Transl Med 3:65ra

Benlian P, de Gennes JL, Dairou F, Hermelin B, Ginon I, Villain E, Lagarde JP, Federspiel MC, Bertrand V, Bernard C, Bereziat G (1996) Phenotypic expression in double heterozygotes for familial hypercholesterolemia and familial defective apolipoprotein B-100. Hum Mutat 7:340-345

Bennett CM, Boye E, Neufeld EJ (2008) Female monozygotic twins discordant for hemophilia A due to nonrandom X-chromosome inactivation. Am J Hematol 83:778-780

Ben-Shachar S, Lanpher B, German JR, Qasaymeh M, Potocki L, Nagamani SC, Franco LM, Malphrus A, Bottenfield GW, Spence JE, Amato S, Rousseau JA, Moghaddam B, Skinner C, Skinner SA, Bernes S, Armstrong N, Shinawi M, Stankiewicz P, Patel A, Cheung SW, Lupski JR, Beaudet AL, Sahoo T (2009) Microdeletion 15q13.3: a locus with incomplete penetrance for autism, mental retardation, and psychiatric disorders. J Med Genet 46:382-388

Berge KE, Haugaa KH, Früh A, Anfinsen OG, Gjesdal K, Siem G, Oyen N, Greve G, Carlsson A, Rognum TO, Hallerud M, Kongsgård E, Amlie JP, Leren TP (2008) Molecular genetic analysis of long QT syndrome in Norway indicating a high prevalence of heterozygous mutation carriers. Scand J Clin Lab Invest 68:362-368

Berlivet S, Moussette S, Ouimet M, Verlaan DJ, Koka V, Al Tuwaijri A, Kwan T, Sinnett D, Pastinen T, Naumova AK (2012) Interaction between genetic and epigenetic variation defines gene expression patterns at the asthma-associated locus 17q12q21 in lymphoblastoid cell lines. Hum Genet 131:1161-1171

Bernardi F, Legnani C, Micheletti F, Lunghi B, Ferraresi P, Palareti G, Biagi R, Marchetti G (1996) A heparin cofactor II mutation (HCII Rimini) combined with factor V Leiden or type I protein C deficiency in two unrelated thrombophilic subjects. Thromb Haemost 76:505-509

Bernardi L, Anfossi M, Gallo M, Geracitano S, Cola R, Puccio G, Curcio SA, Frangipane F, Mirabelli M, Clodomiro A, Di Lorenzo R, Smirne N, Maletta R, Iapaolo D, Bruni AC (2011) PSEN1 and PRNP gene mutations: co-occurrence makes onset very early in a family with FTD phenotype. J Alzheimers Dis 24:415-419

Bertolini S, Pisciotta L, Rabacchi C, Cefalù AB, Noto D, Fasano T, Signori A, Fresa R, Averna M, Calandra S (2013) Spectrum of mutations and phenotypic expression in patients with autosomal dominant hypercholesterolemia identified in Italy. Atherosclerosis 227:342-348

Beutler E (2003) The HFE Cys282Tyr mutation as a necessary but not sufficient cause of clinical hereditary hemochromatosis. Blood 101:3347-3350

Bhuiyan ZA, Jongbloed JD, van der Smagt J, Lombardi PM, Wiesfeld AC, Nelen M, Schouten M, Jongbloed R, Cox MG, van Wolferen M, Rodriguez LM, van Gelder IC, Bikker H, Suurmeijer AJ, van den Berg MP, Mannens MM, Hauer RN, Wilde AA, van Tintelen JP (2009) Desmoglein-2 and desmocollin-2 mutations in Dutch arrhythmogenic right ventricular dysplasia/cardiomypathy patients: results from a multicenter study. Circ Cardiovasc Genet 2:418-427

Biegstraaten M, van Schaik IN, Aerts JM, Langeveld M, Mannens MM, Bour LJ, Sidransky E, Tayebi N, Fitzgibbon E, Hollak CE (2011) A monozygotic twin pair with highly discordant Gaucher phenotypes. Blood Cells Mol Dis 46:39-41

Bisgaard H, Simpson A, Palmer CN, Bønnelykke K, McLean I, Mukhopadhyay S, Pipper CB, Halkjaer LB, Lipworth B, Hankinson J, Woodcock A, Custovic A (2008) Gene-environment interaction in the onset of eczema in infancy: filaggrin lossof-function mutations enhanced by neonatal cat exposure. PLoS Med 5:e131 
Bishop DT, Demenais F, Goldstein AM, Bergman W, Bishop JN, Bressac-de Paillerets B, Chompret A, Ghiorzo P, Gruis N, Hansson J, Harland M, Hayward N, Holland EA, Mann GJ, Mantelli M, Nancarrow D, Platz A, Tucker MA, Melanoma Genetics Consortium (2002) Geographical variation in the penetrance of $C D K N 2 A$ mutations for melanoma. J Natl Cancer Inst 94:894-903

Blair E, Price SJ, Baty CJ, Ostman-Smith I, Watkins H (2001) Mutations in cis can confound genotype-phenotype correlations in hypertrophic cardiomyopathy. J Med Genet 38:385-388

Blau N, van Spronsen FJ, Levy HL (2010) Phenylketonuria. Lancet 376:1417-1427

Blom AM, Bergström F, Edey M, Diaz-Torres M, Kavanagh D, Lampe A, Goodship JA, Strain L, Moghal N, McHugh M, Inward C, Tomson C, Frémeaux-Bacchi V, Villoutreix BO, Goodship TH (2008) A novel non-synonymous polymorphism (p.Arg240His) in C4b-binding protein is associated with atypical hemolytic uremic syndrome and leads to impaired alternative pathway cofactor activity. J Immunol 180:6385-6391

Boeve BF, Boylan KB, Graff-Radford NR, DeJesus-Hernandez M, Knopman DS, Pedraza O, Vemuri P, Jones D, Lowe V, Murray ME, Dickson DW, Josephs KA, Rush BK, Machulda MM, Fields JA, Ferman TJ, Baker M, Rutherford NJ, Adamson J, Wszolek ZK, Adeli A, Savica R, Boot B, Kuntz KM, Gavrilova R, Reeves A, Whitwell J, Kantarci K, Jack CR Jr, Parisi JE, Lucas JA, Petersen RC, Rademakers R (2012) Characterization of frontotemporal dementia and/or amyotrophic lateral sclerosis associated with the GGGGCC repeat expansion in C9ORF72. Brain 135:765-783

Boinot C, Borgel D, Kitzis A, Guicheteau M, Aiach M, Alhenc-Gelas M (2003) Familial thrombophilia is an oligogenetic disease: involvement of the prothrombin G20210A, PROC and PROS gene mutations. Blood Coagul Fibrinolysis 14:191-196

Bökenkamp A, deJong M, van Wijk JA, Block D, van Hagen JM, Ludwig M (2005) R561C missense mutation in the SMARCAL1 gene associated with mild Schimke immuno-osseous dysplasia. Pediatr Nephrol 20:1724-1728

Bolk S, Pelet A, Hofstra RM, Angrist M, Salomon R, Croaker D, Buys CH, Lyonnet S, Chakravarti A (2000) A human model for multigenic inheritance: phenotypic expression in Hirschsprung disease requires both the RET gene and a new 9q31 locus. Proc Natl Acad Sci USA 97:268-273

Bolszak M, Anttonen AK, Komulainen T, Hinttala R, Pakanen S, Sormunen R, Herva R, Lehesjoki AE, Majamaa K, Rantala H, Uusimaa J (2009) Digenic mutations in severe myoclonic epilepsy of infancy. Epilepsy Res 85:300-304

Borrello MG, Aiello A, Peissel B, Rizzetti MG, Mondellini P, Degl'Innocenti D, Catalano V, Gobbo M, Collini P, Bongarzone I, Pierotti MA, Greco A, Seregni E (2011) Functional characterization of the MTC-associated germline RET-K666E mutation: evidence of oncogenic potential enhanced by the G691S polymorphism. Endocr Relat Cancer 18:519-527

Boyko AR, Williamson SH, Indap AR, Degenhardt JD, Hernandez RD, Lohmueller KE, Adams MD, Schmidt S, Sninsky JJ, Sunyaev SR, White TJ, Nielsen R, Clark AG, Bustamante CD (2008) Assessing the evolutionary impact of amino acid mutations in the human genome. PLoS Genet 4:e1000083

Breckpot J, Thienpont B, Arens Y, Tranchevent LC, Vermeesch JR, Moreau Y, Gewillig M, Devriendt K (2011) Challenges of interpreting copy number variation in syndromic and nonsyndromic congenital heart defects. Cytogenet Genome Res 135:251-259

Bremner R, Du DC, Connolly-Wilson MJ, Bridge P, Ahmad KF, Mostachfi H, Rushlow D, Dunn JM, Gallie BL (1997) Deletion of RB exons 24 and 25 causes low-penetrance retinoblastoma. Am J Hum Genet 61:556-570
Brennan P, Hainaut P, Boffetta P (2011) Genetics of lung-cancer susceptibility. Lancet Oncol 12:399-408

Brenner B, Zivelin A, Lanir N, Greengard JS, Griffin JH, Seligsohn U (1996) Venous thromboembolism associated with double heterozygosity for R506Q mutation of factor V and for T298M mutation of protein $\mathrm{C}$ in a large family of a previously described homozygous protein $\mathrm{C}$-deficient newborn with massive thrombosis. Blood 88:877-880

Bresin E, Rurali E, Caprioli J, Sanchez-Corral P, Fremeaux-Bacchi V, Rodriguez de Cordoba S, Pinto S, Goodship TH, Alberti M, Ribes D, Valoti E, Remuzzi G, Noris M, European Working Party on Complement Genetics in Renal Diseases (2013) Combined complement gene mutations in atypical hemolytic uremic syndrome influence clinical phenotype. J Am Soc Nephrol 24:475-486

Bröer S, Bailey CG, Kowalczuk S, Ng C, Vanslambrouck JM, Rodgers H, Auray-Blais C, Cavanaugh JA, Bröer A, Rasko JE (2008) Iminoglycinuria and hyperglycinuria are discrete human phenotypes resulting from complex mutations in proline and glycine transporters. J Clin Invest 118:3881-3892

Bruder CE, Piotrowski A, Gijsbers AA, Andersson R, Erickson S, Diaz de Ståhl T, Menzel U, Sandgren J, von Tell D, Poplawski A, Crowley M, Crasto C, Partridge EC, Tiwari H, Allison DB, Komorowski J, van Ommen GJ, Boomsma DI, Pedersen NL, den Dunnen JT, Wirdefeldt K, Dumanski JP (2008) Phenotypically concordant and discordant monozygotic twins display different DNA copy-number-variation profiles. Am J Hum Genet 82:763-771

Brugnoni R, Kapetis D, Imbrici P, Pessia M, Canioni E, Colleoni L, de Rosbo NK, Morandi L, Cudia P, Gashemi N, Bernasconi P, Desaphy JF, Conte D, Mantegazza R (2013) A large cohort of myotonia congenita probands: novel mutations and a highfrequency mutation region in exons 4 and 5 of the $C L C N 1$ gene. J Hum Genet (in press). doi:10.1038/jhg.2013.58

Bullard JE, Nogee LM (2007) Heterozygosity for $A B C A 3$ mutations modifies the severity of lung disease associated with a surfactant protein C gene (SFTPC) mutation. Pediatr Res 62:176-179

Buratti E, Brindisi A, Pagani F, Baralle FE (2004) Nuclear factor TDP-43 binds to the polymorphic TG repeats in CFTR intron 8 and causes skipping of exon 9: a functional link with disease penetrance. Am J Hum Genet 74:1322-1325

Burdon KP, Wirth MG, Mackey DA, Russell-Eggitt IM, Craig JE, Elder JE, Dickinson JL, Sale MM (2004) A novel mutation in the Connexin 46 gene causes autosomal dominant congenital cataract with incomplete penetrance. J Med Genet 41:e106

Butt C, Zheng H, Randell E, Robb D, Parfrey P, Xie YG (2003) Combined carrier status of prothrombin 20210A and factor XIIIA Leu34 alleles as a strong risk factor for myocardial infarction: evidence of a gene-gene interaction. Blood 101:3037-3041

Caciotti A, Bardelli T, Cunningham J, D’Azzo A, Zammarchi E, Morrone A (2003) Modulating action of the new polymorphism L436F detected in the GLB1 gene of a type-II GM1 gangliosidosis patient. Hum Genet 113:44-50

Cafolla A, D'Andrea G, Baldacci E, Margaglione M, Mazzucconi MG, Foà R (2012) Hereditary protein C deficiency and thrombosis risk: genotype and phenotype relation in a large Italian family. Eur J Haematol 88:336-339

Caglayan AO, Demiryilmaz F, Ozyazgan I, Gumus H. (2010) $M E F V$ gene compound heterozygous mutations in familial Mediterranean fever phenotype: a retrospective clinical and molecular study. Nephrol Dial Transplant 25:2520-2523

Caleshu C, Sakhuja R, Nussbaum RL, Schiller NB, Ursell PC, Eng C, De Marco T, McGlothlin D, Burchard EG, Rame JE (2011) Furthering the link between the sarcomere and primary cardiomyopathies: restrictive cardiomyopathy associated with multiple mutations in genes previously associated with hypertrophic or dilated cardiomyopathy. Am J Med Genet 155A:2229-2235 
Camus D, Shinar Y, Aamar S, Langevitz P, Ben-Zvi I, Livneh A, Lidar M (2012) 'Silent' carriage of two familial Mediterranean fever gene mutations in large families with only a single identified patient. Clin Genet 82:288-291

Canto P, Munguía P, Söderlund D, Castro JJ, Méndez JP (2009) Genetic analysis in patients with Kallmann syndrome: coexistence of mutations in prokineticin receptor 2 and $K A L 1$. J Androl 30:41-45

Caprioli J, Castelletti F, Bucchioni S, Bettinaglio P, Bresin E, Pianetti G, Gamba S, Brioschi S, Daina E, Remuzzi G, Noris M, International Registry of Recurrent and Familial HUS/TTP (2003) Complement factor $\mathrm{H}$ mutations and gene polymorphisms in haemolytic uraemic syndrome: the C-257T, the A2089G and the G2881T polymorphisms are strongly associated with the disease. Hum Mol Genet 12:3385-3395

Cardenas-Rodriguez M, Osborn DP, Irigoín F, Graña M, Romero H, Beales PL, Badano JL (2013) Characterization of CCDC28B reveals its role in ciliogenesis and provides insight to understand its modifier effect on Bardet-Biedl syndrome. Hum Genet 132:91-105

Carvalho C, Bartnik M, Pehlivan D, Fang P, Shen J, Lupski J (2012) Evidence for disease penetrance relating to $\mathrm{CNV}$ size: pelizaeusMerzbacher disease and manifesting carriers with a familial $11 \mathrm{Mb}$ duplication at Xq22. Clin Genet 81:532-541

Carvill GL, Mefford HC (2013) Microdeletion syndromes. Curr Opin Genet Develop 23:1-8

Caspi A, Sugden K, Moffitt TE, Taylor A, Craig IW, Harrington H, McClay J, Mill J, Martin J, Braithwaite A, Poulton R (2003) Influence of life stress on depression: moderation by a polymorphism in the 5-HTT gene. Science 301:386-389

Castaman G, Federici AB, Rodeghiero F, Mannucci PM (2003) von Willebrand's disease in the year 2003: towards the complete identification of gene defects for correct diagnosis and treatment. Haematologica 88:94-108

Castaman G, Bertoncello K, Bernardi M, Eikenboom JC, Budde U, Rodeghiero F (2007) Autosomal recessive von Willebrand disease associated with compound heterozygosity for a novel nonsense mutation (2,908 del C) and the missense mutation C2362F: definite evidence for the non-penetrance of the $\mathrm{C} 2362 \mathrm{~F}$ mutation. Am J Hematol 82:376-380

Castéra L, Sabbagh A, Dehainault C, Michaux D, Mansuet-Lupo A, Patillon B, Lamar E, Aerts I, Lumbroso-Le Rouic L, Couturier J, Stoppa-Lyonnet D, Gauthier-Villars M, Houdayer C (2010) $M D M 2$ as a modifier gene in retinoblastoma. J Natl Cancer Inst 102:1805-1808

Cerutti E, Campagnoli MF, Ferretti M, Garelli E, Crescenzio N, Rosolen A, Chiocchetti A, Lenardo MJ, Ramenghi U, Dianzani U (2007) Co-inherited mutations of Fas and caspase-10 in development of the autoimmune lymphoproliferative syndrome. BMC Immunol 8:28

Chang JC, Wang L, Chen RF, Liu CA (2012) Perinatal gene-gene and gene-environment interactions on IgE production and asthma development. Clin Dev Immunol 2012:270869

Chapman SJ, Hill AV (2012) Human genetic susceptibility to infectious disease. Nat Rev Genet 13:175-188

Chaudru V, Lo MT, Lesueur F, Marian C, Mohamdi H, Laud K, Barrois M, Chompret A, Avril MF, Demenais F, Bressac-de Paillerets B (2009) Protective effect of copy number polymorphism of glutathione S-transferase T1 gene on melanoma risk in presence of $C D K N 2 A$ mutations, $M C 1 R$ variants and host-related phenotypes. Fam Cancer 8:371-377

Chen S, Parmigiani G (2007) Meta-analysis of BRCA1 and BRCA2 penetrance. J Clin Oncol 25:1329-1333

Chen J, Smaoui N, Hammer MB, Jiao X, Riazuddin SA, Harper S, Katsanis N, Riazuddin S, Chaabouni H, Berson EL, Hejtmancik JF (2011) Molecular analysis of Bardet-Biedl syndrome families: report of 21 novel mutations in 10 genes. Invest Ophthalmol Vis Sci 52:5317-5324

Cheng J, Tester DJ, Tan BH, Valdivia CR, Kroboth S, Ye B, January CT, Ackerman MJ, Makielski JC (2011) The common African American polymorphism SCN5A-S1103Y interacts with mutation SCN5A-R680H to increase late $\mathrm{Na}$ current. Physiol Genomics 43:461-466

Cheng WC, Shu WY, Li CY, Tsai ML, Chang CW, Chen CR, Cheng HT, Wang TH, Hsu IC (2012) Intra- and inter-individual variance of gene expression in clinical studies. PLoS ONE 7:e38650

Cheung VG, Spielman RS (2009) Genetics of human gene expression: mapping DNA variants that influence gene expression. Nat Rev Genet 10:595-604

Chhin B, Hatayama M, Bozon D, Ogawa M, Schön P, Tohmonda T, Sassolas F, Aruga J, Valard AG, Chen SC, Bouvagnet P (2007) Elucidation of penetrance variability of a ZIC3 mutation in a family with complex heart defects and functional analysis of ZIC3 mutations in the first zinc finger domain. Hum Mutat 28:563-570

Chiang PW, Fulton AB, Spector E, Hisama FM (2008) Synergistic interaction of the $O C A 2$ and $O C A 3$ genes in a family. Am J Med Genet 146A:2427-2430

Chiang PW, Spector E, McGregor TL (2009) Evidence suggesting digenic inheritance of Waardenburg syndrome type II with ocular albinism. Am J Med Genet 149A:2739-2744

Chiba-Falek O, Kerem E, Shoshani T, Aviram M, Augarten A, Bentur L, Tal A, Tullis E, Rahat A, Kerem B (1998) The molecular basis of disease variability among cystic fibrosis patients carrying the $3,849+10 \mathrm{~kb} \mathrm{C}->$ T mutation. Genomics 53:276-283

Christodoulou K, Wiskin AE, Gibson J, Tapper W, Willis C, Afzal NA, Upstill-Goddard R, Holloway JW, Simpson MA, Beattie RM, Collins A, Ennis S (2013) Next generation exome sequencing of paediatric inflammatory bowel disease patients identifies rare and novel variants in candidate genes. Gut 62:977-984

Chu K, Koller DL, Snyder R, Fishburn T, Lai D, Waguespack SG, Foroud T, Econs MJ (2005) Analysis of variation in expression of autosomal dominant osteopetrosis type 2: searching for modifier genes. Bone 37:655-661

Chung KW, Sunwoo IN, Kim SM, Park KD, Kim WK, Kim TS, Koo H, Cho M, Lee J, Choi BO (2005) Two missense mutations of EGR2 R359W and GJB1 V136A in a Charcot-Marie-Tooth disease family. Neurogenetics 6:159-163

Cigni A, Ledda F, Satta AE (2006) A complex case of renal amyloidosis with a rare co-occurrence of 2 mutations in separate hereditary periodic fever syndrome-related genes. J Nephrol 19:543-549

Čiuladaitè Ž, Kasnauskienè J, Cimbalistiené L, Preikšaitienè E, Patsalis PC, Kučinskas V (2011) Mental retardation and autism associated with recurrent $16 \mathrm{p} 11.2$ microdeletion: incomplete penetrance and variable expressivity. J Appl Genet 52:443-449

Clain J, Fritsch J, Lehmann-Che J, Bali M, Arous N, Goossens M, Edelman A, Fanen P (2001) Two mild cystic fibrosis-associated mutations result in severe cystic fibrosis when combined in cis and reveal a residue important for cystic fibrosis transmembrane conductance regulator processing and function. $\mathrm{J}$ Biol Chem 276:9045-9049

Clementi R, Dagna L, Dianzani U, Dupré L, Dianzani I, Ponzoni M, Cometa A, Chiocchetti A, Sabbadini MG, Rugarli C, Ciceri F, Maccario R, Locatelli F, Danesino C, Ferrarini M, Bregni M (2004) Inherited perforin and Fas mutations in a patient with autoimmune lymphoproliferative syndrome and lymphoma. N Engl J Med 351:1419-1424

Cogan J, Austin E, Hedges L, Womack B, West J, Loyd J, Hamid R (2012) Role of BMPR2 alternative splicing in heritable 
pulmonary arterial hypertension penetrance. Circulation 126:1907-1916

Cohen JC, Kiss RS, Pertsemlidis A, Marcel YL, McPherson R, Hobbs HH (2004) Multiple rare alleles contribute to low plasma levels of HDL cholesterol. Science 305:869-872

Cohen W, Castelli C, Alessi MC, Aillaud MF, Bouvet S, Saut N, Brunet D, Barthet MC, Tregouet DA, Lavigne G, Morange PE (2012) ABO blood group and von Willebrand factor levels partially explained the incomplete penetrance of congenital thrombophilia. Arterioscler Thromb Vasc Biol 32:2021-2028

Cole LW, Sidis Y, Zhang C, Quinton R, Plummer L, Pignatelli D, Hughes VA, Dwyer AA, Raivio T, Hayes FJ, Seminara SB, Huot C, Alos N, Speiser P, Takeshita A, Van Vliet G, Pearce S, Crowley WF Jr, Zhou QY, Pitteloud N (2008) Mutations in prokineticin 2 and prokineticin receptor 2 genes in human gonadotrophin-releasing hormone deficiency: molecular genetics and clinical spectrum. J Clin Endocrinol Metab 93:3551-3559

Constantine CC, Anderson GJ, Vulpe CD, McLaren CE, Bahlo M, Yeap HL, Gertig DM, Osborne NJ, Bertalli NA, Beckman KB, Chen V, Matak P, McKie AT, Delatycki MB, Olynyk JK, English DR, Southey MC, Giles GG, Hopper JL, Allen KJ, Gurrin LC (2009) A novel association between a SNP in CYBRD1 and serum ferritin levels in a cohort study of HFE hereditary haemochromatosis. Br J Haematol 147:140-149

Cooper DN, Chen JM, Ball EV, Howells K, Mort M, Phillips AD, Chuzhanova N, Krawczak M, Kehrer-Sawatzki H, Stenson PD (2010) Genes, mutations, and human inherited disease at the dawn of the age of personalized genomics. Hum Mutat 31:631-655

Cordeiro JM, Perez GJ, Schmitt N, Pfeiffer R, Nesterenko VV, Burashnikov E, Veltmann C, Borggrefe M, Wolpert C, Schimpf R, Antzelevitch C (2010) Overlapping LQT1 and LQT2 phenotype in a patient with long QT syndrome associated with loss-of-function variations in $K C N Q 1$ and $K C N H 2$. Can J Physiol Pharmacol 88:1181-1190

Cordell HJ (2009) Detecting gene-gene interactions that underlie human diseases. Nat Rev Genet 10:392-404

Coventry A, Bull-Otterson LM, Liu X, Clark AG, Maxwell TJ, Crosby J, Hixson JE, Rea TJ, Muzny DM, Lewis LR, Wheeler DA, Sabo A, Lusk C, Weiss KG, Akbar H, Cree A, Hawes AC, Newsham I, Varghese RT, Villasana D, Gross S, Joshi V, Santibanez J, Morgan M, Chang K, Iv WH, Templeton AR, Boerwinkle E, Gibbs R, Sing CF (2010) Deep resequencing reveals excess rare recent variants consistent with explosive population growth. Nat Commun 1:131

Cowley MJ, Cotsapas CJ, Williams RB, Chan EK, Pulvers JN, Liu MY, Luo OJ, Nott DJ, Little PF (2009) Intra- and interindividual genetic differences in gene expression. Mamm Genome 20:281-295

Crotti L, Lundquist AL, Insolia R, Pedrazzini M, Ferrandi C, De Ferrari GM, Vicentini A, Yang P, Roden DM, George AL Jr, Schwartz PJ (2005) KCNH2-K897T is a genetic modifier of latent congenital long-QT syndrome. Circulation 112:1251-1258

Crotti L, Monti MC, Insolia R, Peljto A, Goosen A, Brink PA, Greenberg DA, Schwartz PJ, George AL Jr (2009) NOS1AP is a genetic modifier of the long-QT syndrome. Circulation 120:1657-1663

Croxen R, Hatton C, Shelley C, Brydson M, Chauplannaz G, Oosterhuis H, Vincent A, Newsom-Davis J, Colquhoun D, Beeson D (2002) Recessive inheritance and variable penetrance of slow-channel congenital myasthenic syndromes. Neurology 59:162-168

Cruchaga C, Haller G, Chakraverty S, Mayo K, Vallania FL, Mitra RD, Faber K, Williamson J, Bird T, Diaz-Arrastia R, Foroud TM, Boeve BF, Graff-Radford NR, St. Jean P, Lawson M, Ehm MG, Mayeux R, Goate AM, NIA-LOAD/NCRAD Family Study Consortium (2012) Rare variants in APP, PSEN1 and PSEN2 increase risk for $\mathrm{AD}$ in late-onset Alzheimer's disease families. PLoS ONE 7:e31039

Cullinane CA, Lubinski J, Neuhausen SL, Ghadirian P, Lynch HT, Isaacs C, Weber B, Moller P, Offit K, Kim-Sing C, Friedman E, Randall S, Pasini B, Ainsworth P, Gershoni-Baruch R, Foulkes WD, Klijn J, Tung N, Rennert G, Olopade O, Couch F, Wagner T, Olsson H, Sun P, Weitzel JN, Narod SA (2005) Effect of pregnancy as a risk factor for breast cancer in $B R C A 1 / B R C A 2$ mutation carriers. Int J Cancer 117:988-991

Cuppens H, Lin W, Jaspers M, Costes B, Teng H, Vankeerberghen A, Jorissen M, Droogmans G, Reynaert I, Goossens M, Nilius B, Cassiman JJ (1998) Polyvariant mutant cystic fibrosis transmembrane conductance regulator genes. The polymorphic $(\mathrm{Tg}) \mathrm{m}$ locus explains the partial penetrance of the T5 polymorphism as a disease mutation. J Clin Invest 101:487-496

Custovic A, Marinho S, Simpson A (2012) Gene-environment interactions in the development of asthma and atopy. Expert Rev Respir Med 6:301-308

Członkowska A, Gromadzka G, Chabik G (2009) Monozygotic female twins discordant for phenotype of Wilson's disease. Mov Disord 24:1066-1069

D’Angelo R, Esposito T, Calabrò M, Rinaldi C, Robledo R, Varriale B, Sidoti A (2013) FMO3 allelic variants in Sicilian and Sardinian populations: trimethylaminuria and absence of fishlike body odor. Gene 515:410-415

D’Onofrio M, Ambrosini A, Di Mambro A, Arisi I, Santorelli FM, Grieco GS, Nicoletti F, Nappi G, Pierelli F, Schoenen J, Buzzi MG (2009) The interplay of two single nucleotide polymorphisms in the CACNA1A gene may contribute to migraine susceptibility. Neurosci Lett 453:12-15

Dabell MP, Rosenfeld JA, Bader P, Escobar LF, El-Khechen D, Vallee SE, Dinulos MB, Curry C, Fisher J, Tervo R, Hannibal MC, Siefkas K, Wyatt PR, Hughes L, Smith R, Ellingwood S, Lacassie Y, Stroud T, Farrell SA, Sanchez-Lara PA, Randolph LM, Niyazov D, Stevens CA, Schoonveld C, Skidmore D, MacKay S, Miles JH, Moodley M, Huillet A, Neill NJ, Ellison JW, Ballif BC, Shaffer LG (2013) Investigation of NRXN1 deletions: clinical and molecular characterization. Am J Med Genet A 161:717-731

Dächsel JC, Mata IF, Ross OA, Taylor JP, Lincoln SJ, Hinkle KM, Huerta C, Ribacoba R, Blazquez M, Alvarez V, Farrer MJ (2006) Digenic parkinsonism: investigation of the synergistic effects of PRKN and LRRK2. Neurosci Lett 410:80-84

Dalal D, James C, Devanagondi R, Tichnell C, Tucker A, Prakasa K, Spevak PJ, Bluemke DA, Abraham T, Russell SD, Calkins H, Judge DP (2006) Penetrance of mutations in plakophilin-2 among families with arrhythmogenic right ventricular dysplasia/ cardiomyopathy. J Am Coll Cardiol 48:1416-1424

Daw EW, Chen SN, Czernuszewicz G, Lombardi R, Lu Y, Ma J, Roberts R, Shete S, Marian AJ (2007) Genome-wide mapping of modifier chromosomal loci for human hypertrophic cardiomyopathy. Hum Mol Genet 16:2463-2471

De Cruz R, Horev L, Green J, Babay S, Sladden M, Zlotogorski A, Sinclair R (2012) A novel monilethrix mutation in coil 2A of KRT86 causing autosomal dominant monilethrix with incomplete penetrance. Br J Dermatol 166(Suppl 2):20-26

de la Chapelle A (2009) Genetic predisposition to human disease: allele-specific expression and low-penetrance regulatory loci. Oncogene 28:3345-3348

De Luca A, Sarkozy A, Consoli F, Ferese R, Guida V, Dentici ML, Mingarelli R, Bellacchio E, Tuo G, Limongelli G, Digilio MC, Marino B, Dallapiccola B (2010) Familial transposition of the great arteries caused by multiple mutations in laterality genes. Heart 96:673-677

de Pontual L, Pelet A, Clement-Ziza M, Trochet D, Antonarakis SE, Attie-Bitach T, Beales PL, Blouin JL, Dastot-Le Moal F, Dollfus 
H, Goossens M, Katsanis N, Touraine R, Feingold J, Munnich A, Lyonnet S, Amiel J (2007) Epistatic interactions with a common hypomorphic RET allele in syndromic Hirschsprung disease. Hum Mutat 28:790-796

de Pontual L, Zaghloul NA, Thomas S, Davis EE, McGaughey DM, Dollfus H, Baumann C, Bessling SL, Babarit C, Pelet A, Gascue C, Beales P, Munnich A, Lyonnet S, Etchevers H, Attie-Bitach T, Badano JL, McCallion AS, Katsanis N, Amiel J (2009) Epistasis between RET and $B B S$ mutations modulates enteric innervation and causes syndromic Hirschsprung disease. Proc Natl Acad Sci USA 106:13921-13926

Dedoussis GV, Luo Y, Starremans P, Rossetti S, Ramos AJ, Cantiello HF, Katsareli E, Ziroyannis P, Lamnissou K, Harris PC, Zhou J (2008) Co-inheritance of a PKD1 mutation and homozygous $P K D 2$ variant: a potential modifier in autosomal dominant polycystic kidney disease. Eur J Clin Invest 38:180-190

Dekel B, Metsuyanim S, Goldstein N, Pode-Shakked N, Kovalski Y, Cohen Y, Davidovits M, Anikster Y (2008) Schimke immunoosseous dysplasia: expression of SMARCAL1 in blood and kidney provides novel insight into disease phenotype. Pediatr Res 63:398-403

Delaunay J, Nouyrigat V, Proust A, Schischmanoff PO, Cynober T, Yvart J, Gaillard C, Danos O, Tchernia G (2004) Different impacts of alleles $\alpha$ LEPRA and $\alpha$ LELY as assessed versus a novel, virtually null allele of the SPTA1 gene in trans. Br J Haematol 127:118-122

Del-Castillo-Rueda A, Moreno-Carralero MI, Cuadrado-Grande N, Álvarez-Sala-Walther LA, Enríquez-de-Salamanca R, Méndez M, Morán-Jiménez MJ (2012) Mutations in the HFE, TFR2, and SLC40A1 genes in patients with hemochromatosis. Gene 508:15-20

Denecke J, Brune T, Feldhaus T, Robenek H, Kranz C, Auchus RJ, Agarwal AK, Marquardt T (2006) A homozygous ZMPSTE24 null mutation in combination with a heterozygous mutation in the LMNA gene causes Hutchinson-Gilford progeria syndrome (HGPS): insights into the pathophysiology of HGPS. Hum Mutat 27:524-531

Deugnier Y, Mosser J (2008) Modifying factors of the HFE hemochromatosis phenotype. Expert Rev Gastroenterol Hepatol 2:531-540

Di Pierro E, Brancaleoni V, Moriondo V, Besana V, Cappellini MD (2007) Co-existence of two functional mutations on the same allele of the human ferrochelatase gene in erythropoietic protoporphyria. Clin Genet 71:84-88

Di WL, Hennekam RC, Callard RE, Harper JI (2009) A heterozygous null mutation combined with the G1258A polymorphism of SPINK5 causes impaired LEKTI function and abnormal expression of skin barrier proteins. Br J Dermatol 161:404-412

Dibbens LM, Heron SE, Mulley JC (2007) A polygenic heterogeneity model for common epilepsies with complex genetics. Genes Brain Behav 6:593-597

Dick DM (2011) Gene-environment interaction in psychological traits and disorders. Annu Rev Clin Psychol 7:383-409

Dimas AS, Nica AC, Montgomery SB, Stranger BE, Raj T, Buil A, Giger T, Lappalainen T, Gutierrez-Arcelus M, MuTHER Consortium, McCarthy MI, Dermitzakis ET (2012) Sex-biased genetic effects on gene regulation in humans. Genome Res 22:2368-2375

Ding YC, McGuffog L, Healey S, Friedman E, Laitman Y, PaluchShimon S, Kaufman B; SWE-BRCA, Liljegren A, Lindblom A, Olsson H, Kristoffersson U, Stenmark-Askmalm M, Melin B, Domchek SM, Nathanson KL, Rebbeck TR, Jakubowska A, Lubinski J, Jaworska K, Durda K, Gronwald J, Huzarski T, Cybulski C, Byrski T, Osorio A, Cajal TR, Stavropoulou AV, Benítez J, Hamann U; HEBON, Rookus M, Aalfs CM, de Lange JL, Meijers-Heijboer HE, Oosterwijk JC, van Asperen CJ,
Gómez García EB, Hoogerbrugge N, Jager A, van der Luijt RB; EMBRACE, Easton DF, Peock S, Frost D, Ellis SD, Platte R, Fineberg E, Evans DG, Lalloo F, Izatt L, Eeles R, Adlard J, Davidson R, Eccles D, Cole T, Cook J, Brewer C, Tischkowitz M, Godwin AK, Pathak H; GEMO Study Collaborators, StoppaLyonnet D, Sinilnikova OM, Mazoyer S, Barjhoux L, Léoné M, Gauthier-Villars M, Caux-Moncoutier V, de Pauw A, Hardouin A, Berthet P, Dreyfus H, Ferrer SF, Collonge-Rame MA, Sokolowska J, Buys S, Daly M, Miron A, Terry MB, Chung W, John EM, Southey M, Goldgar D, Singer CF, Tea MK, Gschwantler-Kaulich D, Fink-Retter A, Hansen TV, Ejlertsen B, Johannsson OT, Offit K, Sarrel K, Gaudet MM, Vijai J, Robson M, Piedmonte MR, Andrews L, Cohn D, DeMars LR, DiSilvestro P, Rodriguez G, Toland AE, Montagna M, Agata S, Imyanitov E, Isaacs C, Janavicius R, Lazaro C, Blanco I, Ramus SJ, Sucheston L, Karlan BY, Gross J, Ganz PA, Beattie MS, Schmutzler RK, Wappenschmidt B, Meindl A, Arnold N, Niederacher D, Preisler-Adams S, Gadzicki D, Varon-Mateeva R, Deissler H, Gehrig A, Sutter C, Kast K, Nevanlinna H, Aittomäki K, Simard J; KConFab Investigators, Spurdle AB, Beesley J, Chen X, Tomlinson GE, Weitzel J, Garber JE, Olopade OI, Rubinstein WS, Tung N, Blum JL, Narod SA, Brummel S, Gillen DL, Lindor N, Fredericksen Z, Pankratz VS, Couch FJ, Radice P, Peterlongo P, Greene MH, Loud JT, Mai PL, Andrulis IL, Glendon G, Ozcelik H; OCGN, Gerdes AM, Thomassen M, Jensen UB, Skytte AB, Caligo MA, Lee A, Chenevix-Trench G, Antoniou AC, Neuhausen SL; Consortium of Investigators of Modifiers of BRCA1/2 (CIMBA). (2012) A nonsynonymous polymorphism in IRS1 modifies risk of developing breast and ovarian cancers in BRCAl and ovarian cancer in $B R C A 2$ mutation carriers. Cancer Epidemiol Biomarkers Prev 21:1362-1370

Dipple KM, McCabe ERB (2000) Modifier genes convert "simple" Mendelian disorders to complex traits. Mol Genet Metab 71:43-50

Dobyns WB, Filauro A, Tomson BN, Chan AS, Ho AW, Ting NT, Oosterwijk JC, Ober C (2004) Inheritance of most X-linked traits is not dominant or recessive, just X-linked. Am J Med Genet 129A:136-143

Dodé C, Teixeira L, Levilliers J, Fouveaut C, Bouchard P, Kottler ML, Lespinasse J, Lienhardt-Roussie A, Mathieu M, Moerman A, Morgan G, Murat A, Toublanc JE, Wolczynski S, Delpech M, Petit C, Young J, Hardelin JP (2006) Kallmann syndrome: mutations in the genes encoding prokineticin-2 and prokineticin receptor-2. PLoS Genet 2:e175

Draper N, Walker EA, Bujalska IJ, Tomlinson JW, Chalder SM, Arlt W, Lavery GG, Bedendo O, Ray DW, Laing I, Malunowicz E, White PC, Hewison M, Mason PJ, Connell JM, Shackleton CH, Stewart PM (2003) Mutations in the genes encoding 11betahydroxysteroid dehydrogenase type 1 and hexose-6-phosphate dehydrogenase interact to cause cortisone reductase deficiency. Nat Genet 34:434-439

Drumm ML, Konstan MW, Schluchter MD, Handler A, Pace R, Zou F, Zariwala M, Fargo D, Xu A, Dunn JM, Darrah RJ, Dorfman R, Sandford AJ, Corey M, Zielenski J, Durie P, Goddard K, Yankaskas JR, Wright FA, Knowles MR, Gene Modifier Study Group (2005) Genetic modifiers of lung disease in cystic fibrosis. N Engl J Med 353:1443-1453

Dykes AC, Walker ID, McMahon AD, Islam SI, Tait RC (2001) A study of Protein $\mathrm{S}$ antigen levels in 3,788 healthy volunteers: influence of age, sex and hormone use, and estimate for prevalence of deficiency state. Br J Haematol 113:636-641

Ebermann I, Phillips JB, Liebau MC, Koenekoop RK, Schermer B, Lopez I, Schäfer E, Roux AF, Dafinger C, Bernd A, Zrenner E, Claustres M, Blanco B, Nürnberg G, Nürnberg P, Ruland R, Westerfield M, Benzing T, Bolz HJ (2010) PDZD7 is a modifier 
of retinal disease and a contributor to digenic Usher syndrome. J Clin Invest 120:1812-1823

Eichers ER, Lewis RA, Katsanis N, Lupski JR (2004) Triallelic inheritance: a bridge between Mendelian and multifactorial traits. Ann Med 36:262-272

Ekvall S, Hagenäs L, Allanson J, Annerén G, Bondeson ML (2011) Co-occurring SHOC2 and PTPN11 mutations in a patient with severe/complex Noonan syndrome-like phenotype. Am J Med Genet 155A:1217-1224

El-Hattab AW, Zhang F, Maxim R, Christensen KM, Ward JC, Hines-Dowell S, Scaglia F, Lupski JR, Cheung SW (2010) Deletion and duplication of 15q24: molecular mechanisms and potential modification by additional copy number variants. Genet Med 12:573-586

Elizondo LI, Cho KS, Zhang W, Yan J, Huang C, Huang Y, Choi K, Sloan EA, Deguchi K, Lou S, Baradaran-Heravi A, Takashima H, Lücke T, Quiocho FA, Boerkoel CF (2009) Schimke immuno-osseous dysplasia: SMARCAL1 loss-of-function and phenotypic correlation. J Med Genet 46:49-59

Emery AEH (1986) Risk estimation in autosomal dominant disorders with reduced penetrance. J Med Genet 23:316-318

Emison ES, McCallion AS, Kashuk CS, Bush RT, Grice E, Lin S, Portnoy ME, Cutler DJ, Green ED, Chakravarti A (2005) A common sex-dependent mutation in a RET enhancer underlies Hirschsprung disease risk. Nature 434:857-863

Emison ES, Garcia-Barcelo M, Grice EA, Lantieri F, Amiel J, Burzynski G, Fernandez RM, Hao L, Kashuk C, West K, Miao X, Tam PK, Griseri P, Ceccherini I, Pelet A, Jannot AS, de Pontual L, Henrion-Caude A, Lyonnet S, Verheij JB, Hofstra RM, Antiñolo G, Borrego S, McCallion AS, Chakravarti A (2010) Differential contributions of rare and common, coding and noncoding Ret mutations to multifactorial Hirschsprung disease liability. Am J Hum Genet 87:60-74

Esparza-Gordillo J, Jorge EG, Garrido CA, Carreras L, LópezTrascasa M, Sánchez-Corral P, de Córdoba SR (2006) Insights into hemolytic uremic syndrome: segregation of three independent predisposition factors in a large, multiple affected pedigree. Mol Immunol 43:1769-1775

Esteban Cardeñosa E, de Juan Jiménez I, Palanca Suela S, Chirivella González I, Segura Huerta A, Santaballa Beltran A, Casals El Busto M, Barragán González E, Fuster Lluch O, Bermúdez Edo J, Bolufer Gilabert P (2012) Low penetrance alleles as risk modifiers in familial and sporadic breast cancer. Fam Cancer 11:629-636

Fahim AT, Bowne SJ, Sullivan LS, Webb KD, Williams JT, Wheaton DK, Birch DG, Daiger SP (2011) Allelic heterogeneity and genetic modifier loci contribute to clinical variation in males with $\mathrm{X}$-linked retinitis pigmentosa due to $R P G R$ mutations. PLoS ONE 6:e23021

Fahrner JA, Frazier A, Bachir S, Walsh MF, Applegate CD, Thompson R, Halushka MK, Murphy AM, Gunay-Aygun M (2012) A rasopathy phenotype with severe congenital hypertrophic obstructive cardiomyopathy associated with a PTPN11 mutation and a novel variant in SOS1. Am J Med Genet 158A:1414-1421

Fairley C, Zimran A, Phillips M, Cizmarik M, Yee J, Weinreb N, Packman S (2008) Phenotypic heterogeneity of N370S homozygotes with type I Gaucher disease: an analysis of 798 patients from the ICGG Gaucher Registry. J Inherit Metab Dis 31:738-744

Fan X, Yoshida Y, Honda S, Matsumoto M, Sawada Y, Hattori M, Hisanaga S, Hiwa R, Nakamura F, Tomomori M, Miyagawa S, Fujimaru R, Yamada H, Sawai T, Ikeda Y, Iwata N, Uemura O, Matsukuma E, Aizawa Y, Harada H, Wada H, Ishikawa E, Ashida A, Nangaku M, Miyata T, Fujimura Y (2013) Analysis of genetic and predisposing factors in Japanese patients with atypical hemolytic uremic syndrome. Mol Immunol 54:238-246
Fauser S, Munz M, Besch D (2003) Further support for digenic inheritance in Bardet-Biedl syndrome. J Med Genet 40:e104

Fearnhead NS, Wilding JL, Winney B, Tonks S, Bartlett S, Bicknell DC, Tomlinson IP, Mortensen NJ, Bodmer WF (2004) Multiple rare variants in different genes account for multifactorial inherited susceptibility to colorectal adenomas. Proc Natl Acad Sci USA 101:15992-15997

Fechtel K, Osterbur ML, Kehrer-Sawatzki H, Stenson PD, Cooper DN (2011) Delineating the Hemostaseome as an aid to individualize the analysis of the hereditary basis of thrombotic and bleeding disorders. Hum Genet 130:149-166

Felderbauer P, Hoffmann P, Einwächter H, Bulut K, Ansorge N, Schmitz F, Schmidt WE (2003) A novel mutation of the calcium sensing receptor gene is associated with chronic pancreatitis in a family with heterozygous SPINK1 mutations. BMC Gastroenterol 3:34

Fencl F, Malina M, Stará V, Zieg J, Mixová D, Seeman T, Bláhová K (2012) Discordant expression of a new WT1 gene mutation in a family with monozygotic twins presenting with congenital nephrotic syndrome. Eur J Pediatr 171:121-124

Finch N, Carrasquillo MM, Baker M, Rutherford NJ, Coppola G, Dejesus-Hernandez M, Crook R, Hunter T, Ghidoni R, Benussi L, Crook J, Finger E, Hantanpaa KJ, Karydas AM, Sengdy P, Gonzalez J, Seeley WW, Johnson N, Beach TG, Mesulam M, Forloni G, Kertesz A, Knopman DS, Uitti R, White CL 3rd, Caselli R, Lippa C, Bigio EH, Wszolek ZK, Binetti G, Mackenzie IR, Miller BL, Boeve BF, Younkin SG, Dickson DW, Petersen RC, Graff-Radford NR, Geschwind DH, Rademakers R (2011) TMEM106B regulates progranulin levels and the penetrance of FTLD in GRN mutation carriers. Neurology 76:467-474

Flanagan SE, Xie W, Caswell R, Damhuis A, Vianey-Saban C, Akcay T, Darendeliler F, Bas F, Guven A, Siklar Z, Ocal G, Berberoglu M, Murphy N, O'Sullivan M, Green A, Clayton PE, Banerjee I, Clayton PT, Hussain K, Weedon MN, Ellard S (2013) Nextgeneration sequencing reveals deep intronic cryptic $A B C C 8$ and $H A D H$ splicing founder mutations causing hyperinsulinism by pseudoexon activation. Am J Hum Genet 92:131-136

Floeth M, Bruckner-Tuderman L (1999) Digenic junctional epidermolysis bullosa: mutations in COL17A1 and LAMB3 genes. Am J Hum Genet 65:1530-1537

Font-Llitjós M, Jiménez-Vidal M, Bisceglia L, Di Perna M, de Sanctis L, Rousaud F, Zelante L, Palacín M, Nunes V (2005) New insights into cystinuria: 40 new mutations, genotypephenotype correlation, and digenic inheritance causing partial phenotype. J Med Genet 42:58-68

Forlani G, Zucchini S, Di Rocco A, Di Luzio R, Scipione M, Marasco E, Romeo G, Marchesini G, Mantovani V (2010) Double heterozygous mutations involving both HNF1A/MODY3 and HNF4A/MODY1 genes: a case report. Diabetes Care 33:2336-2338

Formstone CJ, Hallam PJ, Tuddenham EG, Voke J, Layton M, Nicolaides K, Hann IM, Cooper DN (1996) Severe perinatal thrombosis in double and triple heterozygous offspring of a family segregating two independent protein $\mathrm{S}$ mutations and a protein C mutation. Blood 87:3731-3737

Franchini M, Mannucci PM (2009) Multiple gene interaction and modulation of hemostatic balance. Clin Chem Lab Med 47:1455-1460

Frank-Raue K, Rybicki LA, Erlic Z, Schweizer H, Winter A, Milos I, Toledo SP, Toledo RA, Tavares MR, Alevizaki M, Mian C, Siggelkow H, Hüfner M, Wohllk N, Opocher G, Dvořáková S, Bendlova B, Czetwertynska M, Skasko E, Barontini M, Sanso G, Vorländer C, Maia AL, Patocs A, Links TP, de Groot JW, Kerstens MN, Valk GD, Miehle K, Musholt TJ, Biarnes J, Damjanovic S, Muresan M, Wüster C, Fassnacht M, Peczkowska 
M, Fauth C, Golcher H, Walter MA, Pichl J, Raue F, Eng C, Neumann HP, International RET Exon 10 Consortium (2011) Risk profiles and penetrance estimations in multiple endocrine neoplasia type $2 \mathrm{~A}$ caused by germline $R E T$ mutations located in exon 10. Hum Mutat 32:51-58

Friedrich FW, Bausero P, Sun Y, Treszl A, Krämer E, Juhr D, Richard P, Wegscheider K, Schwartz K, Brito D, Arbustini E, Waldenström A, Isnard R, Komajda M, Eschenhagen T, Carrier L, EUROGENE Heart Failure Project (2009) A new polymorphism in human calmodulin III gene promoter is a potential modifier gene for familial hypertrophic cardiomyopathy. Eur Heart J 30:1648-1655

Friedrich K, Rupp C, Hov JR, Steinebrunner N, Weiss K-H, Stiehl A, Brune M, Schaefer PKY, Schemmer P, Sauer P, Schirmacher P, Runz H, Karlsen TH, Stremmel W, Gotthardt DN (2013) A frequent PNPLA3 variant is a sex specific disease modifier in PSC patients with bile duct stenosis. PLoS ONE 8:e58734

Fu W, O'Connor TD, Jun G, Kang HM, Abecasis G, Leal SM, Gabriel S, Altshuler D, Shendure J, Nickerson DA, Bamshad MJ, NHLBI Exome Sequencing Project, Akey JM (2013) Analysis of 6,515 exomes reveals the recent origin of most human proteincoding variants. Nature 493:216-220

Fujita-Jimbo E, Yu ZL, Li H, Yamagata T, Mori M, Momoi T, Momoi MY (2012) Mutation in Parkinson disease-associated, G-protein-coupled receptor 37 (GPR37/PaelR) is related to Autism spectrum disorder. PLoS ONE 7:e51155

Funayama M, Li Y, Tsoi TH, Lam CW, Ohi T, Yazawa S, Uyama E, Djaldetti R, Melamed E, Yoshino H, Imamichi Y, Takashima H, Nishioka K, Sato K, Tomiyama H, Kubo S, Mizuno Y, Hattori N (2008) Familial Parkinsonism with digenic parkin and PINK1 mutations. Mov Disord 23:1461-1465

Furukawa Y, Lang AE, Trugman JM, Bird TD, Hunter A, Sadeh M, Tagawa T, St George-Hyslop PH, Guttman M, Morris LW, Hornykiewicz O, Shimadzu M, Kish SJ (1998) Gender-related penetrance and de novo GTP-cyclohydrolase I gene mutations in dopa-responsive dystonia. Neurology 50:1015-1020

Galassi G, Lamantea E, Invernizzi F, Tavani F, Pisano I, Ferrero I, Palmieri L, Zeviani M (2008) Additive effects of POLG1 and ANT1 mutations in a complex encephalomyopathy. Neuromuscul Disord 18:465-470

Galetzka D, Hansmann T, El Hajj N, Weis E, Irmscher B, Ludwig M, Schneider-Rätzke B, Kohlschmidt N, Beyer V, Bartsch O, Zechner U, Spix C, Haaf T (2012) Monozygotic twins discordant for constitutive BRCA1 promoter methylation, childhood cancer and secondary cancer. Epigenetics 7:47-54

Galvan A, Dragan TA (2009) Nicotine dependence may link the 15q25 locus to lung cancer risk. Carcinogenesis 31:331-333

Gámez-Pozo A, Palacios I, Kontic M, Menéndez I, Camino I, GarcíaMiguel P, Abelairas J, Pestaña A, Alonso J (2007) Pathogenic validation of unique germline intronic variants of $R B 1$ in retinoblastoma patients using minigenes. Hum Mutat 28:1245

Gandrille S, Greengard JS, Alhenc-Gelas M, Juhan-Vague I, Abgrall JF, Jude B, Griffin JH, Aiach M (1995) Incidence of activated protein $\mathrm{C}$ resistance caused by the ARG 506 GLN mutation in factor $\mathrm{V}$ in 113 unrelated symptomatic protein C-deficient patients. The French Network on the behalf of INSERM. Blood 86:219-224

Gan-Or Z, Bar-Shira A, Gurevich T, Giladi N, Orr-Urtreger A (2011) Homozygosity for the MTX1 c.184T >A (p.S63T) alteration modifies the age of onset in GBA-associated Parkinson's disease. Neurogenetics 12:325-332

Garcia-Barcelo MM, Tang CS, Ngan ES, Lui VC, Chen Y, So MT, Leon TY, Miao XP, Shum CK, Liu FQ, Yeung MY, Yuan ZW, Guo WH, Liu L, Sun XB, Huang LM, Tou JF, Song YQ, Chan D, Cheung KM, Wong KK, Cherny SS, Sham PC, Tam PK (2009) Genome-wide association study identifies NRG1 as a susceptibility locus for Hirschsprung's disease. Proc Natl Acad Sci USA 106:2694-2699

Garcia-Garcia AB, Ivorra C, Martinez-Hervas S, Blesa S, Fuentes MJ, Puig O, Martín-de-Llano JJ, Carmena R, Real JT, Chaves FJ (2011) Reduced penetrance of autosomal dominant hypercholesterolemia in a high percentage of families: importance of genetic testing in the entire family. Atherosclerosis 218:423-430

Gasparini P, Rabionet R, Barbujani G, Melçhionda S, Petersen M, Brøndum-Nielsen K, Metspalu A, Oitmaa E, Pisano M, Fortina P, Zelante L, Estivill X (2000) High carrier frequency of the $35 \mathrm{delG}$ deafness mutation in European populations. Genetic Analysis Consortium of GJB2 35delG. Eur J Hum Genet 8:19-23

Gau SS, Liao HM, Hong CC, Chien WH, Chen CH (2012) Identification of two inherited copy number variants in a male with autism supports two-hit and compound heterozygosity models of autism. Am J Med Genet B Neuropsychiatr Genet 159B:710-717

Gehring NH, Frede U, Neu-Yilik G, Hundsdoerfer P, Vetter B, Hentze MW, Kulozik AE (2001) Increased efficiency of mRNA $3^{\prime}$ end formation: a new genetic mechanism contributing to hereditary thrombophilia. Nat Genet 28:389-392

Genuardi M, Klutz M, Devriendt K, Caruso D, Stirpe M, Lohmann DR (2001) Multiple lipomas linked to an $R B 1$ gene mutation in a large pedigree with low penetrance retinoblastoma. Eur J Hum Genet 9:690-694

Germeshausen M, Zeidler C, Stuhrmann M, Lanciotti M, Ballmaier M, Welte K (2010) Digenic mutations in severe congenital neutropenia. Haematologica 95:1207-1210

Gershoni-Baruch R, Brik R, Shinawi M, Livneh A (2002) The differential contribution of $M E F V$ mutant alleles to the clinical profile of familial Mediterranean fever. Eur J Hum Genet 10:145-149

Geyer O, Wolf A, Levinger E, Harari-Shacham A, Walton DS, Shochat C, Korem S, Bercovich D (2011) Genotype/phenotype correlation in primary congenital glaucoma patients from different ethnic groups of the Israeli population. Am J Ophthalmol 151:263-271

Gherasim C, Rosenblatt DS, Banerjee R (2007) Polymorphic background of methionine synthase reductase modulates the phenotype of a disease-causing mutation. Hum Mutat 28:1028-1033

Gianfrancesco F, Rendina D, Di Stefano M, Mingione A, Esposito T, Merlotti D, Gallone S, Magliocca S, Goode A, Formicola D, Morello G, Layfield R, Frattini A, De Filippo G, Nuti R, Searle M, Strazzullo P, Isaia G, Mossetti G, Gennari L (2012) A nonsynonymous TNFRSF11A variation increases NFKB activity and the severity of Paget's disease. J Bone Miner Res 27:443-452

Giess R, Holtmann B, Braga M, Grimm T, Müller-Myhsok B, Toyka KV, Sendtner M (2002) Early onset of severe familial amyotrophic lateral sclerosis with a SOD1 mutation: potential impact of $C N T F$ as a candidate modifier gene. Am $\mathrm{J}$ Hum Genet 70:1277-1286

Gillespie CF, Almli LM, Smith AK, Bradley B, Kerley K, Crain DF, Mercer KB, Weiss T, Phifer J, Tang Y, Cubells JF, Binder EB, Conneely KN, Ressler KJ (2013) Sex dependent influence of a functional polymorphism in steroid 5- $\alpha$-reductase type 2 (SRD5A2) on post-traumatic stress symptoms. Am J Med Genet B Neuropsychiatr Genet 162:283-292

Ginolhac SM, Gad S, Corbex M, Bressac-De-Paillerets B, Chompret A, Bignon YJ, Peyrat JP, Fournier J, Lasset C, Giraud S, Muller D, Fricker JP, Hardouin A, Berthet P, Maugard C, Nogues C, Lidereau R, Longy M, Olschwang S, Toulas C, Guimbaud R, Yannoukakos D, Szabo C, Durocher F, Moisan AM, Simard J, Mazoyer S, Lynch HT, Goldgar D, Stoppa-Lyonnet D, Lenoir 
GM, Sinilnikova OM (2003) BRCA1 wild-type allele modifies risk of ovarian cancer in carriers of $B R C A 1$ germ-line mutations. Cancer Epidemiol Biomarkers Prev 12:90-95

Giráldez MD, Balaguer F, Caldés T, Sanchez-de-Abajo A, GómezFernández N, Ruiz-Ponte C, Muñoz J, Garre P, Gonzalo V, Moreira L, Ocaña T, Clofent J, Carracedo A, Andreu M, Jover R, Llor X, Castells A, Castellví-Bel S, Gastrointestinal Oncology Group of the Spanish Gastroenterological Association (2009) Association of MUTYH and MSH6 germline mutations in colorectal cancer patients. Fam Cancer 8:525-531

Girirajan S, Rosenfeld JA, Coe BP, Parikh S, Friedman N, Goldstein A, Filipink RA, McConnell JS, Angle B, Meschino WS, Nezarati MM, Asamoah A, Jackson KE, Gowans GC, Martin JA, Carmany EP, Stockton DW, Schnur RE, Penney LS, Martin DM, Raskin S, Leppig K, Thiese H, Smith R, Aberg E, Niyazov DM, Escobar LF, El-Khechen D, Johnson KD, Lebel RR, Siefkas K, Ball S, Shur N, McGuire M, Brasington CK, Spence JE, Martin LS, Clericuzio C, Ballif BC, Shaffer LG, Eichler EE (2012) Phenotypic heterogeneity of genomic disorders and rare copy-number variants. N Engl J Med 367:1321-1331

Girolami F, Ho CY, Semsarian C, Baldi M, Will ML, Baldini K, Torricelli F, Yeates L, Cecchi F, Ackerman MJ, Olivotto I (2010) Clinical features and outcome of hypertrophic cardiomyopathy associated with triple sarcomere protein gene mutations. J Am Coll Cardiol 55:1444-1453

Giudicessi JR, Ackerman MJ (2013) Determinants of incomplete penetrance and variable expressivity in heritable cardiac arrhythmia syndromes. Transl Res 161:1-14

Golbus JR, Puckelwartz MJ, Fahrenbach JP, Dellefave-Castillo LM, Wolfgeher D, McNally EM (2012) Population-based variation in cardiomyopathy genes. Circ Cardiovasc Genet 5:391-399

Goldfarb LG, Petersen RB, Tabaton M, Brown P, LeBlanc AC, Montagna P, Cortelli P, Julien J, Vital C, Pendelbury WW (1992) Fatal familial insomnia and familial Creutzfeldt-Jakob disease: disease phenotype determined by a DNA polymorphism. Science 258:806-808

Goode DL, Cooper GM, Schmutz J, Dickson M, Gonzales E, Tsai M, Karra K, Davydov E, Batzoglou S, Myers RM, Sidow A (2010) Evolutionary constraint facilitates interpretation of genetic variation in resequenced human genomes. Genome Res 20:301-310

Goodin DS (2012) The genetic and environmental bases of complex human-disease: extending the utility of twin-studies. PLoS ONE $7: \mathrm{e} 47875$

Gordon L, Joo JH, Andronikos R, Ollikainen M, Wallace EM, Umstad MP, Permezel M, Oshlack A, Morley R, Carlin JB, Saffery R, Smyth GK, Craig JM (2011) Expression discordance of monozygotic twins at birth: effect of intrauterine environment and a possible mechanism for fetal programming. Epigenetics 6:579-592

Gordon L, Joo JE, Powell JE, Ollikainen M, Novakovic B, Li X, Andronikos R, Cruickshank MN, Conneely KN, Smith AK, Alisch RS, Morley R, Visscher PM, Craig JM, Saffery R (2012) Neonatal DNA methylation profile in human twins is specified by a complex interplay between intrauterine environmental and genetic factors, subject to tissue-specific influence. Genome Res 22:1395-1406

Gorlov IP, Gorlova OY, Frazier ML, Spitz MR, Amos CI (2011) Evolutionary evidence of the effect of rare variants on disease etiology. Clin Genet 79:199-206

Gouya L, Puy H, Lamoril J, Da Silva V, Grandchamp B, Nordmann Y, Deybach JC (1999) Inheritance in erythropoietic protoporphyria: a common wild-type ferrochelatase allelic variant with low expression accounts for clinical manifestation. Blood 93:2105-2110

Gouya L, Puy H, Robreau AM, Bourgeois M, Lamoril J, Da Silva V, Grandchamp B, Deybach JC (2002) The penetrance of dominant erythropoietic protoporphyria is modulated by expression of wildtype FECH. Nat Genet 30:27-28

Gouya L, Martin-Schmitt C, Robreau AM, Austerlitz F, Da Silva V, Brun P, Simonin S, Lyoumi S, Grandchamp B, Beaumont C, Puy H, Deybach JC (2006) Contribution of a common singlenucleotide polymorphism to the genetic predisposition for erythropoietic protoporphyria. Am J Hum Genet 78:2-14

Grabowski M, Zimprich A, Lorenz-Depiereux B, Kalscheuer V, Asmus F, Gasser T, Meitinger T, Strom TM (2003) The epsilonsarcoglycan gene $(S G C E)$, mutated in myoclonus-dystonia syndrome, is maternally imprinted. Eur $\mathbf{J}$ Hum Genet 11:138-144

Gracia-Aznarez FJ, Fernandez V, Pita G, Peterlongo P, Dominguez O, de la Hoya M, Duran M, Osorio A, Moreno L, Gonzalez-Neira A, Rosa-Rosa JM, Sinilnikova O, Mazoyer S, Hopper J, Lazaro C, Southey M, Odefrey F, Manoukian S, Catucci I, Caldes T, Lynch HT, Hilbers FSM, van Asperen CJ, Vasen HFA, Golgar D, Radice P, Devilee P, Benitez J (2013) Whole exome sequencing suggests much of non-BRCA1/BRCA2 familial breast cancer is due to moderate and low penetrance susceptibility alleles. PLoS ONE 8:e55681

Granados-Riveron JT, Pope M, Bu'lock FA, Thornborough C, Eason J, Setchfield K, Ketley A, Kirk EP, Fatkin D, Feneley MP, Harvey RP, Brook JD (2012) Combined mutation screening of NKX2-5, GATA4, and TBX5 in congenital heart disease: multiple heterozygosity and novel mutations. Congenit Heart Dis 7:151-159

Grocock CJ, Rebours V, Delhaye MN, Andrén-Sandberg A, Weiss FU, Mountford R, Harcus MJ, Niemczyck E, Vitone LJ, Dodd S, Jørgensen MT, Ammann RW, Schaffalitzky de Muckadell O, Butler JV, Burgess P, Kerr B, Charnley R, Sutton R, Raraty MG, Devière J, Whitcomb DC, Neoptolemos JP, Lévy P, Lerch MM, Greenhalf W, European Registry of Hereditary Pancreatitis and Pancreatic Cancer (2010) The variable phenotype of the p.A16 V mutation of cationic trypsinogen (PRSS1) in pancreatitis families. Gut 59:357-363

Groman JD, Hefferon TW, Casals T, Bassas L, Estivill X, Des Georges M, Guittard C, Koudova M, Fallin MD, Nemeth K, Fekete G, Kadasi L, Friedman K, Schwarz M, Bombieri C, Pignatti PF, Kanavakis E, Tzetis M, Schwartz M, Novelli G, D'Apice MR, Sobczynska-Tomaszewska A, Bal J, Stuhrmann M, Macek M Jr, Claustres M, Cutting GR (2004) Variation in a repeat sequence determines whether a common variant of the cystic fibrosis transmembrane conductance regulator gene is pathogenic or benign. Am J Hum Genet 74:176-179

Gros-Louis F, Andersen PM, Dupre N, Urushitani M, Dion P, Souchon F, D'Amour M, Camu W, Meininger V, Bouchard JP, Rouleau GA, Julien JP (2009) Chromogranin B P413L variant as risk factor and modifier of disease onset for amyotrophic lateral sclerosis. Proc Natl Acad Sci USA 106:21777-21782

Gruber R, Wilson NJ, Smith FJ, Grabher D, Steinwender L, Fritsch PO, Schmuth M (2009) Increased pachyonychia congenita severity in patients with concurrent keratin and filaggrin mutations. Br J Dermatol 161:1391-1395

Grundberg E, Small KS, Hedman ̊K, Nica AC, Buil A, Keildson S, Bell JT, Yang TP, Meduri E, Barrett A, Nisbett J, Sekowska M, Wilk A, Shin SY, Glass D, Travers M, Min JL, Ring S, Ho K, Thorleifsson G, Kong A, Thorsteindottir U, Ainali C, Dimas AS, Hassanali N, Ingle C, Knowles D, Krestyaninova M, Lowe CE, Di Meglio P, Montgomery SB, Parts L, Potter S, Surdulescu G, Tsaprouni L, Tsoka S, Bataille V, Durbin R, Nestle FO, O'Rahilly S, Soranzo N, Lindgren CM, Zondervan KT, Ahmadi KR, Schadt EE, Stefansson K, Smith GD, McCarthy MI, Deloukas P, Dermitzakis ET, Spector TD, Multiple Tissue Human Expression Resource (MuTHER) Consortium (2012) Mapping cis- and trans-regulatory effects across multiple tissues in twins. Nat Genet 44:1084-1089 
Grundy CB, Melissari E, Lindo V, Scully MF, Kakkar VV, Cooper $\mathrm{DN}$ (1991) Late-onset homozygous protein C deficiency. Lancet 338:575-576

Guerrero R, Vernia S, Sanz R, Abreu-Rodríguez I, Almaraz C, García-Hoyos M, Michelucci R, Tassinari CA, Riguzzi P, Nobile C, Sanz P, Serratosa JM, Gómez-Garre P (2011) A PTG variant contributes to a milder phenotype in Lafora disease. PLoS ONE 6:e21294

Gui J, Wang T, Trump D, Zimmer T, Lei M (2010) Mutation-specific effects of polymorphism H558R in SCN5A-related sick sinus syndrome. J Cardiovasc Electrophysiol 21:564-573

Hamid R, Cogan JD, Hedges LK, Austin E, Phillips JA 3rd, Newman JH, Loyd JE (2009a) Penetrance of pulmonary arterial hypertension is modulated by the expression of normal BMPR2 allele. Hum Mutat 30:649-654

Hamid R, Phillips JA 3rd, Holladay C, Cogan JD, Austin ED, Backeljauw PF, Travers SH, Patton JG (2009b) A molecular basis for variation in clinical severity of isolated growth hormone deficiency type II. J Clin Endocrinol Metab 94:4728-4734

Hamza TH, Chen H, Hill-Burns EM, Rhodes SL, Montimurro J, Kay DM, Tenesa A, Kusel VI, Sheehan P, Eaaswarkhanth M, Yearout D, Samii A, Roberts JW, Agarwal P, Bordelon Y, Park Y, Wang L, Gao J, Vance JM, Kendler KS, Bacanu SA, Scott WK, Ritz B, Nutt J, Factor SA, Zabetian CP, Payami H (2011) Genome-wide gene-environment study identifies glutamate receptor gene GRIN2A as a Parkinson's disease modifier gene via interaction with coffee. PLoS Genet 7:e1002237

Harada Y, Sutomo R, Sadewa AH, Akutsu T, Takeshima Y, Wada H, Matsuo M, Nishio H (2002) Correlation between SMN2 copy number and clinical phenotype of spinal muscular atrophy: three SMN2 copies fail to rescue some patients from the disease severity. J Neurol 249:1211-1219

Harbour JW (2001) Molecular basis of low-penetrance retinoblastoma. Arch Ophthalmol 119:1699-1704

Harlid S, Ivarsson MI, Butt S, Grzybowska E, Eyfjörd JE, Lenner P, Försti A, Hemminki K, Manjer J, Dillner J, Carlson J (2012) Combined effect of low-penetrant SNPs on breast cancer risk. $\mathrm{Br}$ J Cancer 106:389-396

Harries LW, Locke JM, Shields B, Hanley NA, Hanley KP, Steele A, Njølstad PR, Ellard S, Hattersley AT (2008) The diabetic phenotype in HNF4A mutation carriers is moderated by the expression of HNF4A isoforms from the P1 promoter during fetal development. Diabetes 57:1745-1752

Hayashida M, Yamada H, Yamazaki S, Nomura H, Yoshimura K, Kitahara O, Momose K, Kubo K, Kurihara M, Hamasaki N (2003) Combined protein $\mathrm{C}$ and protein $\mathrm{S}$ deficiency in a family with repetitive thromboembolism and segregated gene mutations. Intern Med 42:268-272

Healy DG, Falchi M, O'Sullivan SS, Bonifati V, Durr A, Bressman S, Brice A, Aasly J, Zabetian CP, Goldwurm S, Ferreira JJ, Tolosa E, Kay DM, Klein C, Williams DR, Marras C, Lang AE, Wszolek ZK, Berciano J, Schapira AH, Lynch T, Bhatia KP, Gasser T, Lees AJ, Wood NW, International LRRK2 Consortium (2008) Phenotype, genotype, and worldwide genetic penetrance of LRRK2-associated Parkinson's disease: a case-control study. Lancet Neurol 7:583-590

Heidemann S, Fischer C, Engel C, Fischer B, Harder L, Schlegelberger B, Niederacher D, Goecke TO, Doelken SC, Dikow N, Jonat W, Morlot S, Schmutzler RC, Arnold NK (2012) Double heterozygosity for mutations in BRCA1 and BRCA2 in German breast cancer patients: implications on test strategies and clinical management. Breast Cancer Res Treat 134:1229-1239

Hellman U, Alarcon F, Lundgren HE, Suhr OB, Bonaiti-Pellié C, Planté-Bordeneuve V (2008) Heterogeneity of penetrance in familial amyloid polyneuropathy, ATTR Val30Met, in the Swedish population. Amyloid 15:181-186

Hensen EF, Jansen JC, Siemers MD, Oosterwijk JC, Vriends AH, Corssmit EP, Bayley JP, van der Mey AG, Cornelisse CJ, Devilee P (2010) The Dutch founder mutation SDHD.D92Y shows a reduced penetrance for the development of paragangliomas in a large multigenerational family. Eur J Hum Genet 18:62-66

Hes FJ, Weiss MM, Woortman SA, de Miranda NF, van Bunderen PA, Bonsing BA, Stokkel MP, Morreau H, Romijn JA, Jansen JC, Vriends AH, Bayley JP, Corssmit EP (2010) Low penetrance of a $S D H B$ mutation in a large Dutch paraganglioma family. BMC Med Genet 11:92

Hidalgo-Bravo A, Pompa-Mera EN, Kofman-Alfaro S, GonzalezBonilla CR, Zenteno JC (2005) A novel filamin A D203Y mutation in a female patient with otopalatodigital type 1 syndrome and extremely skewed $\mathrm{X}$ chromosome inactivation. Am J Med Genet A 136:190-193

Hirose Y, Chiba K, Karasugi T, Nakajima M, Kawaguchi Y, Mikami Y, Furuichi T, Mio F, Miyake A, Miyamoto T, Ozaki K, Takahashi A, Mizuta H, Kubo T, Kimura T, Tanaka T, Toyama Y, Ikegawa S (2008) A functional polymorphism in THBS2 that affects alternative splicing and MMP binding is associated with lumbar-disc herniation. Am J Hum Genet 82:1122-1129

Hmani-Aifa M, Benzina Z, Zulfiqar F, Dhouib H, Shahzadi A, Ghorbel A, Rebaï A, Söderkvist P, Riazuddin S, Kimberling WJ, Ayadi H (2009) Identification of two new mutations in the GPR 98 and the PDE6B genes segregating in a Tunisian family. Eur J Hum Genet 17:474-482

Ho YY, Ionita-Laza I, Ottman R (2012) Domain-dependent clustering and genotype-phenotype analysis of LGI1 mutations in ADPEAF. Neurology 78:563-568

Hodapp JA, Carter GT, Lipe HP, Michelson SJ, Kraft GH, Bird TD (2006) Double trouble in hereditary neuropathy: concomitant mutations in the $P M P-22$ gene and another gene produce novel phenotypes. Arch Neurol 63:112-117

Hoefele J, Wolf MT, O'Toole JF, Otto EA, Schultheiss U, Dêschenes G, Attanasio M, Utsch B, Antignac C, Hildebrandt F (2007) Evidence of oligogenic inheritance in nephronophthisis. J Am Soc Nephrol 18:2789-2795

Hoffmann F, Lohse P, Stojanov S, Shin YS, Renner ED, Kéry A, Zellerer S, Belohradsky BH (2005) Identification of a novel mevalonate kinase gene mutation in combination with the common $M V K$ V377I substitution and the low-penetrance TNFRSF1A R92Q mutation. Eur J Hum Genet 13:510-512

Holmgren G, Wikström L, Lundgren HE, Suhr OB (2004) Discordant penetrance of the trait for familial amyloidotic polyneuropathy in two pairs of monozygotic twins. J Intern Med 256:453-456

Horowitz M, Pasmanik-Chor M, Borochowitz Z, Falik-Zaccai T, Heldmann K, Carmi R, Parvari R, Beit-Or H, Goldman B, Peleg L, Levy-Lahad E, Renbaum P, Legum S, Shomrat R, Yeger H, Benbenisti D, Navon R, Dror V, Shohat M, Magal N, Navot N, Eyal N (1998) Prevalence of glucocerebrosidase mutations in the Israeli Ashkenazi Jewish population. Hum Mutat 12:240-244

Hosak L, Silhan P, Hosakova J (2012) Genomic copy number variations: a breakthrough in our knowledge on schizophrenia etiology? Neuro Endocrinol Lett 33:183-190

Houlston RS, Peto J (2004) The search for low-penetrance cancer susceptibility alleles. Oncogene 23:6471-6476

Hsiao TL, Vitkup D (2008) Role of duplicate genes in robustness against deleterious human mutations. PLoS Genet 4:e1000014

Hu Z, Jin G, Wang L, Chen F, Wang X, Shen H (2007) MDM2 promoter polymorphism SNP309 contributes to tumor susceptibility: evidence from 21 case-control studies. Cancer Epidemiol Biomarkers Prev 16:2717-2723 
Hu RM, Tan BH, Orland KM, Valdivia CR, Peterson A, Pu J, Makielski JC (2013) Digenic inheritance novel mutations in SCN5A and SNTA1 increase late INa contributing to LQT syndrome. Am J Physiol Heart Circ Physiol 304:H994-H1001

Hunault M, Arbini AA, Lopaciuk S, Carew JA, Bauer KA (1997) The Arg353Gln polymorphism reduces the level of coagulation factor VII. In vivo and in vitro studies. Arterioscler Thromb Vasc Biol 17:2825-2829

Hung RJ, McKay JD, Gaborieau V, Boffetta P, Hashibe M, Zaridze D, Mukeria A, Szeszenia-Dabrowska N, Lissowska J, Rudnai P, Fabianova E, Mates D, Bencko V, Foretova L, Janout V, Chen C, Goodman G, Field JK, Liloglou T, Xinarianos G, Cassidy A, McLaughlin J, Liu G, Narod S, Krokan HE, Skorpen F, Elvestad MB, Hveem K, Vatten L, Linseisen J, Clavel-Chapelon F, Vineis P, Bueno-de-Mesquita HB, Lund E, Martinez C, Bingham S, Rasmuson T, Hainaut P, Riboli E, Ahrens W, Benhamou S, Lagiou P, Trichopoulos D, Holcátová I, Merletti F, Kjaerheim K, Agudo A, Macfarlane G, Talamini R, Simonato L, Lowry R, Conway DI, Znaor A, Healy C, Zelenika D, Boland A, Delepine M, Foglio M, Lechner D, Matsuda F, Blanche H, Gut I, Heath S, Lathrop M, Brennan P (2008) A susceptibility locus for lung cancer maps to nicotinic acetylcholine receptor subunit genes on 15q25. Nature 452:633-637

Hung CC, Lin SY, Lee CN, Chen CP, Lin SP, Chao MC, Chiou SS, $\mathrm{Su}$ YN (2011) Low penetrance of retinoblastoma for p.V654L mutation of the $R B 1$ gene. BMC Med Genet 12:76

Hunter DJ (2005) Gene-environment interactions in human diseases. Nat Rev Genet 6:287-298

Huntington Study Group COHORT Investigators (2012) Characterization of a large group of individuals with Huntington disease and their relatives enrolled in the COHORT study. PLoS ONE 7:e29522

Hutchinson S, Furger A, Halliday D, Judge DP, Jefferson A, Dietz HC, Firth H, Handford PA (2003) Allelic variation in normal human FBN1 expression in a family with Marfan syndrome: a potential modifier of phenotype? Hum Mol Genet 12:2269-2276

Hutter CM, Chang-Claude J, Slattery ML, Pflugeisen BM, Lin Y, Duggan D, Nan H, Lemire M, Rangrej J, Figueiredo JC, Jiao S, Harrison TA, Liu Y, Chen LS, Stelling DL, Warnick GS, Hoffmeister M, Küry S, Fuchs CS, Giovannucci E, Hazra A, Kraft P, Hunter DJ, Gallinger S, Zanke BW, Brenner H, Frank B, Ma J, Ulrich CM, White E, Newcomb PA, Kooperberg C, LaCroix AZ, Prentice RL, Jackson RD, Schoen RE, Chanock SJ, Berndt SI, Hayes RB, Caan BJ, Potter JD, Hsu L, Bézieau S, Chan AT, Hudson TJ, Peters U (2012) Characterization of geneenvironment interactions for colorectal cancer susceptibility loci. Cancer Res 72:2036-2044

Iatropoulos P, Daina E, Mele C, Maranta R, Remuzzi G, Noris M (2012) Discordant phenotype in monozygotic twins with renal coloboma syndrome and a $P A X 2$ mutation. Pediatr Nephrol 27:1989-1993

Ingles J, Doolan A, Chiu C, Seidman J, Seidman C, Semsarian C (2005) Compound and double mutations in patients with hypertrophic cardiomyopathy: implications for genetic testing and counselling. J Med Genet 42:e59

Island ML, Jouanolle AM, Mosser A, Deugnier Y, David V, Brissot P, Loréal O (2009) A new mutation in the hepcidin promoter impairs its BMP response and contributes to a severe phenotype in $H F E$-related hemochromatosis. Haematologica 94:720-724

Itoh H, Shimizu W, Hayashi K, Yamagata K, Sakaguchi T, Ohno S, Makiyama T, Akao M, Ai T, Noda T, Miyazaki A, Miyamoto Y, Yamagishi M, Kamakura S, Horie M (2010) Long QT syndrome with compound mutations is associated with a more severe phenotype: a Japanese multicenter study. Heart Rhythm 7:1411-1418
Jackson CE, Fischer RE, Hsu AP, Anderson SM, Choi Y, Wang J, Dale JK, Fleisher TA, Middelton LA, Sneller MC, Lenardo MJ, Straus SE, Puck JM (1999) Autoimmune lymphoproliferative syndrome with defective Fas: genotype influences penetrance. Am J Hum Genet 64:1002-1014

Jacquemont S, Hagerman RJ, Leehey MA, Hall DA, Levine RA, Brunberg JA, Zhang L, Jardini T, Gane LW, Harris SW, Herman K, Grigsby J, Greco CM, Berry-Kravis E, Tassone F, Hagerman PJ (2004) Penetrance of the fragile $X$-associated tremor/ataxia syndrome in a premutation carrier population. JAMA 291:460-469

Jacolot S, Le Gac G, Scotet V, Quere I, Mura C, Férec C (2004) $H A M P$ as a modifier gene that increases the phenotypic expression of the HFE pC282Y homozygous genotype. Blood 103:2835-2840

Jan de Beur S, Ding C, Germain-Lee E, Cho J, Maret A, Levine MA (2003) Discordance between genetic and epigenetic defects in pseudohypoparathyroidism type $1 \mathrm{~b}$ revealed by inconsistent loss of maternal imprinting at GNAS1. Am J Hum Genet 73:314-322

Jendrzejewski J, He H, Radomska HS, Li W, Tomsic J, Liyanarachchi S, Davuluri RV, Nagy R, de la Chapelle A (2012) The polymorphism rs 944289 predisposes to papillary thyroid carcinoma through a large intergenic noncoding RNA gene of tumor suppressor type. Proc Natl Acad Sci USA 109:8646-8651

Jensen MK, Havndrup O, Christiansen M, Andersen PS, Diness B, Axelsson A, Skovby F, Køber L, Bundgaard H (2013) Penetrance of hypertrophic cardiomyopathy in children and adolescents: a 12-year follow-up study of clinical screening and predictive genetic testing. Circulation 127:48-54

Jernström H, Lubinski J, Lynch HT, Ghadirian P, Neuhausen S, Isaacs C, Weber BL, Horsman D, Rosen B, Foulkes WD, Friedman E, Gershoni-Baruch R, Ainsworth P, Daly M, Garber J, Olsson H, Sun P, Narod SA (2004) Breast-feeding and the risk of breast cancer in BRCA1 and BRCA2 mutation carriers. J Natl Cancer Inst 96:1094-1098

Jiang Q, Ho YY, Hao L, Nichols Berrios C, Chakravarti A (2011) Copy number variants in candidate genes are genetic modifiers of Hirschsprung disease. PLoS ONE 6:e21219

Jin ZB, Mandai M, Yokota T, Higuchi K, Ohmori K, Ohtsuki F, Takakura S, Itabashi T, Wada Y, Akimoto M, Ooto S, Suzuki T, Hirami Y, Ikeda H, Kawagoe N, Oishi A, Ichiyama S, Takahashi M, Yoshimura N, Kosugi S (2008) Identifying pathogenic genetic background of simplex or multiplex retinitis pigmentosa patients: a large scale mutation screening study. J Med Genet 45:465-472

Joergensen MT, Brusgaard K, Crüger DG, Gerdes AM, Schaffalitzky de Muckadell OB (2010) Genetic, epidemiological, and clinical aspects of hereditary pancreatitis: a population-based cohort study in Denmark. Am J Gastroenterol 105:1876-1883

Johansen CT, Hegele RA (2012) Allelic and phenotypic spectrum of plasma triglycerides. Biochim Biophys Acta 1821:833-842

Johansen CT, Wang J, McIntyre AD, Martins RA, Ban MR, Lanktree MB, Huff MW, Péterfy M, Mehrabian M, Lusis AJ, Kathiresan S, Anand SS, Yusuf S, Lee AH, Glimcher LH, Cao H, Hegele RA (2012) Excess of rare variants in non-genome-wide association study candidate genes in patients with hypertriglyceridemia. Circ Cardiovasc Genet 5:66-72

Kajiwara K, Berson EL, Dryja TP (1994) Digenic retinitis pigmentosa due to mutations at the unlinked peripherin/RDS and ROM1 loci. Science 264:1604-1608

Kallberg H, Padyukov L, Plenge RM, Ronnelid J, Gregersen PK, van der Helm-van Mil AH, Toes RE, Huizinga TW, Klareskog L, Alfredsson L, Epidemiological Investigation of Rheumatoid Arthritis study group (2007) Gene-gene and gene-environment interactions involving $H L A-D R B 1, P T P N 22$, and smoking in two subsets of rheumatoid arthritis. Am J Hum Genet 80:867-875 
Kaminsky ZA, Tang T, Wang SC, Ptak C, Oh GH, Wong AH, Feldcamp LA, Virtanen C, Halfvarson J, Tysk C, McRae AF, Visscher PM, Montgomery GW, Gottesman II, Martin NG, Petronis A (2009) DNA methylation profiles in monozygotic and dizygotic twins. Nat Genet 41:240-245

Kaneko H, Kawamoto N, Asano T, Mabuchi Y, Horikoshi H, Teramoto T, Matsui E, Kondo M, Fukao T, Kasahara K, Kondo $\mathrm{N}$ (2005) Leaky phenotype of X-linked agammaglobulinaemia in a Japanese family. Clin Exp Immunol 140:520-523

Karges B, Bergmann C, Scholl K, Heinze E, Rasche FM, Zerres K, Debatin KM, Wabitsch M, Karges W (2007) Digenic inheritance of hepatocyte nuclear factor- $1 \alpha$ and $-1 \beta$ with maturity-onset diabetes of the young, polycystic thyroid, and urogenital malformations. Diabetes Care 30:1613-1614

Kaski DN, Pennington C, Beck J, Poulter M, Uphill J, Bishop MT, Linehan JM, O'Malley C, Wadsworth JD, Joiner S, Knight RS, Ironside JW, Brandner S, Collinge J, Mead S (2011) Inherited prion disease with 4-octapeptide repeat insertion: disease requires the interaction of multiple genetic risk factors. Brain 134:1829-1838

Katsanis N, Ansley SJ, Badano JL, Eichers ER, Lewis RA, Hoskins BE, Scambler PJ, Davidson WS, Beales PL, Lupski JR (2001) Triallelic inheritance in Bardet-Biedl syndrome, a Mendelian recessive disorder. Science 293:2256-2259

Kaur-Knudsen D, Bojesen SE, Tybjærg-Hansen A, Nordestgaard BG (2011) Nicotinic acetylcholine receptor polymorphism, smoking behavior, and tobacco-related cancer and lung and cardiovascular diseases: a cohort study. J Clin Oncol 29:2875-2882

Kauwe JS, Bertelsen S, Mayo K, Cruchaga C, Abraham R, Hollingworth P, Harold D, Owen MJ, Williams J, Lovestone S, Morris JC, Goate AM, Alzheimer's Disease Neuroimaging Initiative (2010) Suggestive synergy between genetic variants in $T F$ and $H F E$ as risk factors for Alzheimer's disease. Am J Med Genet B Neuropsychiatr Genet 153B:955-959

Kawamura S, Ikeda Y, Tomita K, Watanabe N, Seki K (2004) A family of hypokalemic periodic paralysis with CACNA1S gene mutation showing incomplete penetrance in women. Intern Med 43:218-222

Ke Q, Luo B, Qi M, Du Y, Wu W (2013) Gender differences in penetrance and phenotype in hypokalemic periodic paralysis. Muscle Nerve 47:41-45

Keenan BT, Shulman JM, Chibnik LB, Raj T, Tran D, Sabuncu MR, Alzheimer's Disease Neuroimaging Initiative, Allen AN, Corneveaux JJ, Hardy JA, Huentelman MJ, Lemere CA, Myers AJ, Nicholson-Weller A, Reiman EM, Evans DA, Bennett DA, De Jager PL (2012) A coding variant in $C R 1$ interacts with APOE$\varepsilon 4$ to influence cognitive decline. Hum Mol Genet 21:2377-2388

Kelberman D, Islam L, Holder SE, Jacques TS, Calvas P, Hennekam RC, Nischal KK, Sowden JC (2011) Digenic inheritance of mutations in FOXC1 and PITX2: correlating transcription factor function and Axenfeld-Rieger disease severity. Hum Mutat 32:1144-1152

Kellermayer R, Keller M, Ratajczak P, Richardson E, Harangi F, Mérei E, Melegh B, Kosztolányi G, Richard G (2005) Bigenic connexin mutations in a patient with hidrotic ectodermal dysplasia. Eur J Dermatol 15:75-79

Kenna KP, McLaughlin RL, Hardiman O, Bradley DG (2013) Using reference databases of genetic variation to evaluate the potential pathogenicity of candidate disease variants. Hum Mutat 34:836-841

Kentsis A, Anewalt R, Ganguly A, Allen JB, Neufeld EJ (2009) Discordant haemophilia $\mathrm{A}$ in male siblings due to a de novo mutation on a familial missense mutant allele. Haemophilia 15:971-972

Kerlin BA, Yan SB, Isermann BH, Brandt JT, Sood R, Basson BR, Joyce DE, Weiler H, Dhainaut JF (2003) Survival advantage associated with heterozygous factor $\mathrm{V}$ Leiden mutation in patients with severe sepsis and in mouse endotoxemia. Blood 102:3085-3092

Khalid A, Finkelstein S, Thompson B, Kelly L, Hanck C, Godfrey TE, Whitcomb DC (2006) A 93 year old man with the PRSS1 R122H mutation, low SPINK1 expression, and no pancreatitis: insights into phenotypic non-penetrance. Gut 55:728-731

Khanna H, Davis EE, Murga-Zamalloa CA, Estrada-Cuzcano A, Lopez I, den Hollander AI, Zonneveld MN, Othman MI, Waseem N, Chakarova CF, Maubaret C, Diaz-Font A, MacDonald I, Muzny DM, Wheeler DA, Morgan M, Lewis LR, Logan CV, Tan PL, Beer MA, Inglehearn CF, Lewis RA, Jacobson SG, Bergmann C, Beales PL, Attié-Bitach T, Johnson CA, Otto EA, Bhattacharya SS, Hildebrandt F, Gibbs RA, Koenekoop RK, Swaroop A, Katsanis N (2009) A common allele in RPGRIP1L is a modifier of retinal degeneration in ciliopathies. Nat Genet 41:739-745

Khayat M, Hardouf H, Zlotogora J, Shalev SA (2010) High carriers frequency of an apparently ancient founder mutation p.Tyr322X in the ERCC8 gene responsible for Cockayne syndrome among Christian Arabs in Northern Israel. Am J Med Genet 152A:3091-3094

Kiesewetter S, Macek M Jr, Davis C, Curristin SM, Chu CS, Graham C, Shrimpton AE, Cashman SM, Tsui LC, Mickle J, Amos J, Highsmith WE, Shuber A, Witt DR, Crystal RG, Cutting GR (1993) A mutation in CFTR produces different phenotypes depending on chromosomal background. Nat Genet 5:274-278

Kilpeläinen TO, Qi L, Brage S, Sharp SJ, Sonestedt E, Demerath E, Ahmad T, Mora S, Kaakinen M, Sandholt CH, Holzapfel C, Autenrieth CS, Hyppönen E, Cauchi S, He M, Kutalik Z, Kumari M, Stančáková A, Meidtner K, Balkau B, Tan JT, Mangino M, Timpson NJ, Song Y, Zillikens MC, Jablonski KA, Garcia ME, Johansson S, Bragg-Gresham JL, Wu Y, van Vliet-Ostaptchouk JV, Onland-Moret NC, Zimmermann E, Rivera NV, Tanaka T, Stringham HM, Silbernagel G, Kanoni S, Feitosa MF, Snitker S, Ruiz JR, Metter J, Larrad MT, Atalay M, Hakanen M, Amin N, Cavalcanti-Proença C, Grøntved A, Hallmans G, Jansson JO, Kuusisto J, Kähönen M, Lutsey PL, Nolan JJ, Palla L, Pedersen O, Pérusse L, Renström F, Scott RA, Shungin D, Sovio U, Tammelin TH, Rönnemaa T, Lakka TA, Uusitupa M, Rios MS, Ferrucci L, Bouchard C, Meirhaeghe A, Fu M, Walker M, Borecki IB, Dedoussis GV, Fritsche A, Ohlsson C, Boehnke M, Bandinelli S, van Duijn CM, Ebrahim S, Lawlor DA, Gudnason V, Harris TB, Sørensen TI, Mohlke KL, Hofman A, Uitterlinden AG, Tuomilehto J, Lehtimäki T, Raitakari O, Isomaa B, Njølstad PR, Florez JC, Liu S, Ness A, Spector TD, Tai ES, Froguel P, Boeing H, Laakso M, Marmot M, Bergmann S, Power C, Khaw KT, Chasman D, Ridker P, Hansen T, Monda KL, Illig T, Järvelin MR, Wareham NJ, Hu FB, Groop LC, Orho-Melander M, Ekelund U, Franks PW, Loos RJ (2011) Physical activity attenuates the influence of $F T O$ variants on obesity risk: a metaanalysis of 218,166 adults and 19,268 children. PLoS Med 8:e1001116

Klassen T, Davis C, Goldman A, Burgess D, Chen T, Wheeler D, McPherson J, Bourquin T, Lewis L, Villasana D, Morgan M, Muzny D, Gibbs R, Noebels J (2011) Exome sequencing of ion channel genes reveals complex profiles confounding personal risk assessment in epilepsy. Cell 145:1036-1048

Klei L, Sanders SJ, Murtha MT, Hus V, Lowe JK, Willsey AJ, Moreno-De-Luca D, Yu TW, Fombonne E, Geschwind D, Grice DE, Ledbetter DH, Lord C, Mane SM, Lese Martin C, Martin DM, Morrow EM, Walsh CA, Melhem NM, Chaste P, Sutcliffe JS, State MW, Cook EH Jr, Roeder K, Devlin B (2012) Common genetic variants, acting additively, are a major source of risk for autism. Mol Autism 3:9

Kleinle S, Lang R, Fischer GF, Vierhapper H, Waldhauser F, Födinger M, Baumgartner-Parzer SM (2009) Duplications of the 
functional CYP21A2 gene are primarily restricted to Q318X alleles: evidence for a founder effect. J Clin Endocrinol Metab 94:3954-3958

Klengel T, Mehta D, Anacker C, Rex-Haffner M, Pruessner JC, Pariante CM, Pace TW, Mercer KB, Mayberg HS, Bradley B, Nemeroff CB, Holsboer F, Heim CM, Ressler KJ, Rein T, Binder EB (2013) Allele-specific FKBP5 DNA demethylation mediates gene-childhood trauma interactions. Nat Neurosci 16:33-41

Klopocki E, Lohan S, Doelken SC, Stricker S, Ockeloen CW, Thiele Soares, de Aguiar R, Lezirovitz K, Mingroni Netto RC, Jamsheer A, Shah H, Kurth I, Habenicht R, Warman M, Devriendt K, Kordass U, Hempel M, Rajab A, Mäkitie O, Naveed M, Radhakrishna U, Antonarakis SE, Horn D, Mundlos S (2012) Duplications of BHLHA9 are associated with ectrodactyly and tibia hemimelia inherited in non-Mendelian fashion. $\mathrm{J}$ Med Genet 49:119-125

Klutz M, Brockmann D, Lohmann DR (2002) A parent-of-origin effect in two families with retinoblastoma is associated with a distinct splice mutation in the RBI gene. Am J Hum Genet 71:174-179

Knoll B, Hach-Wunderle V, Rieger S, Häring D, Mannhalter C (2001) Combined occurrence of a heterozygous missense mutation in the protein $\mathrm{C}$ gene and allelic exclusion of one protein $\mathrm{S}$ allele leading to severe venous thrombosis. Thromb Res 103:3-8

Kock N, Naismith TV, Boston HE, Ozelius LJ, Corey DP, Breakefield XO, Hanson PI (2006) Effects of genetic variations in the dystonia protein torsin A: identification of polymorphism at residue 216 as protein modifier. Hum Mol Genet 15:1355-1364

Koeijvoets KC, Mooijaart SP, Dallinga-Thie GM, Defesche JC, Steyerberg EW, Westendorp RG, Kastelein JJ, van Hagen PM, Sijbrands EJ (2009) Complement factor H Y402H decreases cardiovascular disease risk in patients with familial hypercholesterolaemia. Eur Heart J 30:618-623

Koeleman BP, Reitsma PH, Allaart CF, Bertina RM (1994) Activated protein $\mathrm{C}$ resistance as an additional risk factor for thrombosis in protein C-deficient families. Blood 84:1031-1035

Koeleman BP, van Rumpt D, Hamulyák K, Reitsma PH, Bertina RM (1995) Factor V Leiden: an additional risk factor for thrombosis in protein S deficient families? Thromb Haemost 74:580-583

Kontorovich T, Levy A, Korostishevsky M, Nir U, Friedman E (2010) Single nucleotide polymorphisms in miRNA binding sites and miRNA genes as breast/ovarian cancer risk modifiers in Jewish high-risk women. Int J Cancer 127:589-597

Koren-Michowitz M, Friedman E, Gershoni-Baruch R, Brok-Simoni F, Patael Y, Rechavi G, Amariglio N (2005) Coinheritance of BRCA1 and BRCA2 mutations with Fanconi anemia and Bloom syndrome mutations in Ashkenazi Jewish population: possible role in risk modification for cancer development. Am J Hematol 78:203-206

Kowalewski C, Hamada T, Wozniak K, Kawano Y, Szczecinska W, Yasumoto S, Schwartz RA, Hashimoto T (2007) A novel autosomal partially dominant mutation designated G476D in the keratin 5 gene causing epidermolysis bullosa simplex WeberCockayne type: a family study with a genetic twist. Int J Mol Med 20:75-78

Kratzke RA, Otterson GA, Hogg A, Coxon AB, Geradts J, Cowell JK, Kaye FJ (1994) Partial inactivation of the RB product in a family with incomplete penetrance of familial retinoblastoma and benign retinal tumors. Oncogene 9:1321-1326

Krayenbuehl PA, Hersberger M, Truninger K, Müllhaupt B, Maly FE, Bargetzi M, Schulthess G (2010) Toll-like receptor 4 gene polymorphism modulates phenotypic expression in patients with hereditary hemochromatosis. Eur J Gastroenterol Hepatol $22: 835-841$

Krüger S, Silber AS, Engel C, Görgens H, Mangold E, Pagenstecher C, Holinski-Feder E, von Knebel Doeberitz M, Moeslein G,
Dietmaier W, Stemmler S, Friedl W, Rüschoff J, Schackert HK, German Hereditary Non-Polyposis Colorectal Cancer Consortium (2005) Arg462Gln sequence variation in the prostatecancer-susceptibility gene RNASEL and age of onset of hereditary non-polyposis colorectal cancer: a case-control study. Lancet Oncol 6:566-572

Krüger S, Engel C, Bier A, Silber AS, Görgens H, Mangold E, Pagenstecher C, Holinski-Feder E, von Knebel Doeberitz M, Royer-Pokora B, Dechant S, Pox C, Rahner N, Müller A, Schackert HK, German HNPCC Consortium (2007) The additive effect of p53 Arg72Pro and RNASEL Arg462Gln genotypes on age of disease onset in Lynch syndrome patients with pathogenic germline mutations in MSH2 or MLH1. Cancer Lett 252:55-64

Kryukov GV, Pennacchio LA, Sunyaev SR (2007) Most rare missense alleles are deleterious in humans: implications for complex disease and association studies. Am J Hum Genet 80:727-739

Kubo T, Kitaoka H, Okawa M, Baba Y, Hirota T, Hayato K, Yamasaki N, Matsumura Y, Otsuka H, Arimura T, Kimura A, Doi YL (2011) Genetic screening and double mutation in Japanese patients with hypertrophic cardiomyopathy. Circ J 75:2654-2659

Kujovich JL (2011) Factor V Leiden thrombophilia. Genet Med 13:1-16

Kumar V, Westra HJ, Karjalainen J, Zhernakova DV, Esko T, Hrdlickova B, Almeida R, Zhernakova A, Reinmaa E, Võsa U, Hofker MH, Fehrmann RS, Fu J, Withoff S, Metspalu A, Franke L, Wijmenga C (2013) Human disease-associated genetic variation impacts large intergenic non-coding RNA expression. PLoS Genet 9:e1003201

Küry S, Buecher B, Robiou-du-Pont S, Scoul C, Colman H, Le Neel T, Le Houérou C, Faroux R, Ollivry J, Lafraise B, Chupin LD, Sébille V, Bézieau S (2008) Low-penetrance alleles predisposing to sporadic colorectal cancers: a French case-controlled genetic association study. BMC Cancer 8:326

Kwok JB, Raskin S, Morgan G, Antoniuk SA, Bruk I, Schofield PR (2001) Mutations in the glycine receptor alpha1 subunit (GLRA1) gene in hereditary hyperekplexia pedigrees: evidence for non-penetrance of mutation Y279C. J Med Genet 38:E17

Kwong AK, Fung CW, Chan SY, Wong VC (2012) Identification of SCN1A and PCDH19 mutations in Chinese children with Dravet syndrome. PLoS ONE 7:e41802

Lachmann RH, Grant IR, Halsall D, Cox TM (2004) Twin pairs showing discordance of phenotype in adult Gaucher's disease. QJM 97:199-204

Lahtinen AM, Lehtonen A, Kaartinen M, Toivonen L, Swan H, Widén E, Lehtonen E, Lehto VP, Kontula K (2008) Plakophilin2 missense mutations in arrhythmogenic right ventricular cardiomyopathy. Int J Cardiol 126:92-100

Lahtinen AM, Marjamaa A, Swan H, Kontula K (2011) KCNE1 D85N polymorphism - a sex-specific modifier in type 1 long QT syndrome? BMC Med Genet 12:11

Lappalainen T, Montgomery SB, Nica AC, Dermitzakis ET (2011) Epistatic selection between coding and regulatory variation in human evolution and disease. Am J Hum Genet 89:459-463

LaRusch J, Barmada MM, Solomon S, Whitcomb DC (2012) Whole exome sequencing identifies multiple, complex etiologies in an idiopathic hereditary pancreatitis kindred. JOP 13:258-262

Latourelle JC, Sun M, Lew MF, Suchowersky O, Klein C, Golbe LI, Mark MH, Growdon JH, Wooten GF, Watts RL, Guttman M, Racette BA, Perlmutter JS, Ahmed A, Shill HA, Singer C, Goldwurm S, Pezzoli G, Zini M, Saint-Hilaire MH, Hendricks AE, Williamson S, Nagle MW, Wilk JB, Massood T, Huskey KW, Laramie JM, DeStefano AL, Baker KB, Itin I, Litvan I, Nicholson G, Corbett A, Nance M, Drasby E, Isaacson S, Burn DJ, Chinnery PF, Pramstaller PP, Al-hinti J, Moller AT, Ostergaard K, Sherman SJ, Roxburgh R, Snow B, Slevin JT, 
Cambi F, Gusella JF, Myers RH (2008) The Gly2019Ser mutation in $L R R K 2$ is not fully penetrant in familial Parkinson's disease: the GenePD study. BMC Med 6:32

Lavie O, Narod S, Lejbkowicz F, Dishon S, Goldberg Y, Gemer O, Rennert G (2011) Double heterozygosity in the BRCA1 and $B R C A 2$ genes in the Jewish population. Ann Oncol 22:964-966

Law MJ, Lower KM, Voon HP, Hughes JR, Garrick D, Viprakasit V, Mitson M, De Gobbi M, Marra M, Morris A, Abbott A, Wilder SP, Taylor S, Santos GM, Cross J, Ayyub H, Jones S, Ragoussis J, Rhodes D, Dunham I, Higgs DR, Gibbons RJ (2010) ATR-X syndrome protein targets tandem repeats and influences allele-specific expression in a size-dependent manner. Cell 143:367-378

Lazarin GA, Haque IS, Nazareth S, Iori K, Patterson AS, Jacobson JL, Marshall JR, Seltzer WK, Patrizio P, Evans EA, Srinivasan BS (2013) An empirical estimate of carrier frequencies for $400+$ causal Mendelian variants: results from an ethnically diverse clinical sample of 23,453 individuals. Genet Med 15:178-186

Le Ber I, Camuzat A, Guillot-Noel L, Hannequin D, Lacomblez L, Golfier V, Puel M, Martinaud O, Deramecourt V, RivaudPechoux S, Millecamps S, Vercelletto M, Couratier P, Sellal F, Pasquier F, Salachas F, Thomas-Antérion C, Didic M, Pariente J, Seilhean D, Ruberg M, Wargon I, Blanc F, Camu W, Michel BF, Berger E, Sauvée M, Thauvin-Robinet C, Mondon K, TournierLasserve E, Goizet C, Fleury M, Viennet G, Verpillat P, Meininger V, Duyckaerts C, Dubois B, Brice A (2013) C9ORF72 repeat expansions in the frontotemporal dementias spectrum of diseases: a flow-chart for genetic testing. J Alzheimers Dis 34:485-499

Lebo RV, Grody WW (2007) Variable penetrance and expressivity of the splice altering $5 \mathrm{~T}$ sequence in the cystic fibrosis gene. Genet Test 11:32-44

Lee JM, Ramos EM, Lee JH, Gillis T, Mysore JS, Hayden MR, Warby SC, Morrison P, Nance M, Ross CA, Margolis RL, Squitieri F, Orobello S, Di Donato S, Gomez-Tortosa E, Ayuso C, Suchowersky O, Trent RJ, McCusker E, Novelletto A, Frontali M, Jones R, Ashizawa T, Frank S, Saint-Hilaire MH, Hersch SM, Rosas HD, Lucente D, Harrison MB, Zanko A, Abramson RK, Marder K, Sequeiros J, Paulsen JS, PREDICTHD study of the Huntington Study Group (HSG), Landwehrmeyer GB; REGISTRY study of the European Huntington's Disease Network, Myers RH; HD-MAPS Study Group, MacDonald ME, Gusella JF, COHORT study of the HSG (2012a) CAG repeat expansion in Huntington disease determines age at onset in a fully dominant fashion. Neurology 78:690-695

Lee Y, Gamazon ER, Rebman E, Lee Y, Lee S, Dolan ME, Cox NJ, Lussier YA (2012) Variants affecting exon skipping contribute to complex traits. PLoS Genet 8:e1002998

Leegte B, van der Hout AH, Deffenbaugh AM, Bakker MK, Mulder IM, ten Berge A, Leenders EP, Wesseling J, de Hullu J, Hoogerbrugge N, Ligtenberg MJ, Ardern-Jones A, Bancroft E, Salmon A, Barwell J, Eeles R, Oosterwijk JC (2005) Phenotypic expression of double heterozygosity for $B R C A 1$ and $B R C A 2$ germline mutations. J Med Genet 42:e20

Lefévre SH, Chauveinc L, Stoppa-Lyonnet D, Michon J, Lumbroso L, Berthet P, Frappaz D, Dutrillaux B, Chevillard S, Malfoy B (2002) A T to C mutation in the polypyrimidine tract of the exon 9 splicing site of the $R B 1$ gene responsible for low penetrance hereditary retinoblastoma. J Med Genet 39:E21

Leistritz DF, Pepin MG, Schwarze U, Byers PH (2011) COL3A1 haploinsufficiency results in a variety of Ehlers-Danlos syndrome type IV with delayed onset of complications and longer life expectancy. Genet Med 13:717-722

Lekarev O, Tafuri K, Lane AH, Zhu G, Nakamoto JM, Buller-Burckle AM, Wilson TA, New MI (2013) Erroneous prenatal diagnosis of congenital adrenal hyperplasia owing to a duplication of the CYP21A2 gene. J Perinatol 33:76-78

Lerch MM, Mayerle J, Aghdassi AA, Budde C, Nitsche C, Sauter G, Persike M, Günther A, Simon P, Weiss FU (2010) Advances in the etiology of chronic pancreatitis. Dig Dis 28:324-329

Li FF, Li QQ, Tan ZX, Zhang SY, Liu J, Zhao EY, Yu GC, Zhou J, Zhang LM, Liu SL (2012) A novel mutation in CACNA1S gene associated with hypokalemic periodic paralysis which has a genderdifference in the penetrance. J Mol Neurosci 46:378-383

Li-Chang HH, Driman DK, Levin H, Siu VM, Scanlan NL, Buckley K, Cairney AE, Ainsworth PJ (2013) Colorectal cancer in a 9-year-old due to combined EPCAM and MSH2 germline mutations: case report of a unique genotype and immunophenotype. J Clin Pathol (in press)

Lim KP, Yip SP, Cheung SC, Leung KW, Lam ST, To CH (2009) Novel PRPF31 and PRPH2 mutations and co-occurrence of $P R P F 31$ and $R H O$ mutations in Chinese patients with retinitis pigmentosa. Arch Ophthalmol 127:784-790

Liu JY, Dai X, Sheng J, Cui X, Wang X, Jiang X, Tu X, Tang Z, Bai Y, Liu M, Wang QK (2008) Identification and functional characterization of a novel splicing mutation in RP gene PRPF31. Biochem Biophys Res Commun 367:420-426

Liu XZ, Yuan Y, Yan D, Ding EH, Ouyang XM, Fei Y, Tang W, Yuan H, Chang Q, Du LL, Zhang X, Wang G, Ahmad S, Kang DY, Lin X, Dai P (2009) Digenic inheritance of non-syndromic deafness caused by mutations at the gap junction proteins $C \times 26$ and $C x 31$. Hum Genet 125:53-62

Lo HS, Wang Z, Hu Y, Yang HH, Gere S, Buetow KH, Lee MP (2003) Allelic variation in gene expression is common in the human genome. Genome Res 13:1855-1862

Loewen CJ, Moritz OL, Molday RS (2001) Molecular characterization of peripherin- 2 and rom- 1 mutants responsible for digenic retinitis pigmentosa. J Biol Chem 276:22388-22396

Lohmueller KE, Indap AR, Schmidt S, Boyko AR, Hernandez RD, Hubisz MJ, Sninsky JJ, White TJ, Sunyaev SR, Nielsen R, Clark AG, Bustamante CD (2008) Proportionally more deleterious genetic variation in European than in African populations. Nature 451:994-997

Lopes LR, Zekavati A, Syrris P, Hubank M, Giambartolomei C, Dalageorgou C, Jenkins S, McKenna W, Uk10k Consortium Consortium, Plagnol V, Elliott PM (2013) Genetic complexity in hypertrophic cardiomyopathy revealed by high-throughput sequencing. J Med Genet 50:228-239

Lorenzon A, Beffagna G, Bauce B, De Bortoli M, Li Mura IE, Calore M, Dazzo E, Basso C, Nava A, Thiene G, Rampazzo A (2013) Desmin mutations and arrhythmogenic right ventricular cardiomyopathy. Am J Cardiol 111:400-405

Löwik M, Levtchenko E, Westra D, Groenen P, Steenbergen E, Weening J, Lilien M, Monnens L, van den Heuvel L (2008) Bigenic heterozygosity and the development of steroid-resistant focal segmental glomerulosclerosis. Nephrol Dial Transplant 23:3146-3151

Lupski JR, Belmont JW, Boerwinkle E, Gibbs RA (2011) Clan genomics and the complex architecture of human disease. Cell 147:32-43

MacArthur DG, Balasubramanian S, Frankish A, Huang N, Morris J, Walter K, Jostins L, Habegger L, Pickrell JK, Montgomery SB, Albers CA, Zhang ZD, Conrad DF, Lunter G, Zheng H, Ayub Q, DePristo MA, Banks E, Hu M, Handsaker RE, Rosenfeld JA, Fromer M, Jin M, Mu XJ, Khurana E, Ye K, Kay M, Saunders GI, Suner MM, Hunt T, Barnes IH, Amid C, Carvalho-Silva DR, Bignell AH, Snow C, Yngvadottir B, Bumpstead S, Cooper DN, Xue Y, Romero IG, 1000 Genomes Project Consortium, Wang J, Li Y, Gibbs RA, McCarroll SA, Dermitzakis ET, Pritchard JK, Barrett JC, Harrow J, Hurles ME, Gerstein MB, Tyler-Smith C 
(2012) A systematic survey of loss-of-function variants in human protein-coding genes. Science 335:823-828

Machens A, Schaaf L, Karges W, Frank-Raue K, Bartsch DK, Rothmund M, Schneyer U, Goretzki P, Raue F, Dralle H (2007) Age-related penetrance of endocrine tumours in multiple endocrine neoplasia type 1 (MEN1): a multicentre study of 258 gene carriers. Clin Endocrinol 67:613-622

Majounie E, Renton AE, Mok K, Dopper EG, Waite A, Rollinson S, Chiò A, Restagno G, Nicolaou N, Simon-Sanchez J, van Swieten JC, Abramzon Y, Johnson JO, Sendtner M, Pamphlett R, Orrell RW, Mead S, Sidle KC, Houlden H, Rohrer JD, Morrison KE, Pall H, Talbot K, Ansorge O; Chromosome 9-ALS/FTD Consortium; French research network on FTLD/FTLD/ALS; ITALSGEN Consortium, Hernandez DG, Arepalli S, Sabatelli M, Mora G, Corbo M, Giannini F, Calvo A, Englund E, Borghero G, Floris GL, Remes AM, Laaksovirta H, McCluskey L, Trojanowski JQ, Van Deerlin VM, Schellenberg GD, Nalls MA, Drory VE, Lu CS, Yeh TH, Ishiura H, Takahashi Y, Tsuji S, Le Ber I, Brice A, Drepper C, Williams N, Kirby J, Shaw P, Hardy J, Tienari PJ, Heutink P, Morris HR, Pickering-Brown S, Traynor BJ (2011) Frequency of the C9orf72 hexanucleotide repeat expansion in patients with amyotrophic lateral sclerosis and frontotemporal dementia: a cross-sectional study. Lancet Neurol 11:323-330

Maller J, George S, Purcell S, Fagerness J, Altshuler D, Daly MJ, Seddon JM (2006) Common variation in three genes, including a noncoding variant in $\mathrm{CFH}$, strongly influences risk of age-related macular degeneration. Nat Genet 38:1055-1059

Maloney JP, Stearman RS, Bull TM, Calabrese DW, Tripp-Addison ML, Wick MJ, Broeckel U, Robbins IM, Wheeler LA, Cogan JD, Loyd JE (2012) Loss-of-function thrombospondin-1 mutations in familial pulmonary hypertension. Am J Physiol Lung Cell Mol Physiol 302:L541-L554

Mankad A, Taniguchi T, Cox B, Akkari Y, Rathbun RK, Lucas L, Bagby G, Olson S, D'Andrea A, Grompe M (2006) Natural gene therapy in monozygotic twins with Fanconi anemia. Blood 107:3084-3090

Marchani EE, Bird TD, Steinbart EJ, Rosenthal E, Yu CE, Schellenberg GD, Wijsman EM (2010) Evidence for three loci modifying age-at-onset of Alzheimer's disease in early onset PSEN2 families. Am J Med Genet B Neuropsychiatr Genet 153B:1031-1041

Margolin DH, Kousi M, Chan YM, Lim ET, Schmahmann JD, Hadjivassiliou M, Hall JE, Adam I, Dwyer A, Plummer L, Aldrin SV, O'Rourke J, Kirby A, Lage K, Milunsky A, Milunsky JM, Chan J, Hedley-Whyte ET, Daly MJ, Katsanis N, Seminara SB (2013) Ataxia, dementia, and hypogonadotropism caused by disordered ubiquitination. N Engl J Med 368:1992-2003

Marras C, Klein C, Lang AE, Wakutani Y, Moreno D, Sato C, Yip E, Munhoz RP, Lohmann K, Djarmati A, Bi A, Rogaeva E (2010) LRRK2 and Parkin mutations in a family with parkinsonism-lack of genotype-phenotype correlation. Neurobiol Aging 31:721-722

Marth GT, Yu F, Indap AR, Garimella K, Gravel S, Leong WF, TylerSmith C, Bainbridge M, Blackwell T, Zheng-Bradley X, Chen Y, Challis D, Clarke L, Ball EV, Cibulskis K, Cooper DN, Fulton B, Hartl C, Koboldt D, Muzny D, Smith R, Sougnez C, Stewart C, Ward A, Yu J, Xue Y, Altshuler D, Bustamante CD, Clark AG, Daly M, DePristo M, Flicek P, Gabriel S, Mardis E, Palotie A, Gibbs R, 1000 Genomes Project (2011) The functional spectrum of low-frequency coding variation. Genome Biol 12:R84

Martin N, Zügge K, Brandt R, Friebel D, Janssen B, Zimmerhackl LB (2003) Discordant clinical manifestations in monozygotic twins with the identical mutation in the TSC2 gene. Clin Genet 63:427-430

Martinelli N, Trabetti E, Pinotti M, Olivieri O, Sandri M, Friso S, Pizzolo F, Bozzini C, Caruso PP, Cavallari U, Cheng S, Pignatti
PF, Bernardi F, Corrocher R, Girelli D (2008) Combined effect of hemostatic gene polymorphisms and the risk of myocardial infarction in patients with advanced coronary atherosclerosis. PLoS ONE 3:e1523

Martinez SL, Kolodner RD (2010) Functional analysis of human mismatch repair gene mutations identifies weak alleles and polymorphisms capable of polygenic interactions. Proc Natl Acad Sci USA 107:5070-5075

Martino D, Gajos A, Gallo V, Cif L, Coubes P, Tinazzi M, Schneider SA, Fiorio M, Zorzi G, Nardocci N, Ben-Shlomo Y, Edwards MJ, Bhatia KP (2013) Extragenetic factors and clinical penetrance of DYT1 dystonia: an exploratory study. J Neurol 260:1081-1086

Masson E, Le Maréchal C, Levy P, Chuzhanova N, Ruszniewski P, Cooper DN, Chen JM, Férec C (2007) Co-inheritance of a novel deletion of the entire SPINK1 gene with a CFTR missense mutation (L997F) in a family with chronic pancreatitis. Mol Genet Metab 92:168-175

Mathias A, Moss AJ, Lopes CM, Barsheshet A, McNitt S, Zareba W, Robinson JL, Locati EH, Ackerman MJ, Benhorin J, Kaufman ES, Platonov PG, Qi M, Shimizu W, Towbin JA, Vincent GM, Wilde AA, Zhang L, Goldenberg I (2013) Prognostic implications of mutation-specific QTc standard deviation in congenital long QT syndrome. Heart Rhythm 10:720-725

Matsuo T, Okamoto S, Izumi Y, Hosokawa A, Takegawa T, Fukui H, Tun Z, Honda K, Matoba R, Tatsumi K, Amino N (2000) A novel mutation of the $K A L 1$ gene in monozygotic twins with Kallmann syndrome. Eur J Endocrinol 143:783-787

Matsuzaki H, Wang PH, Hu J, Rava R, Fu GK (2009) High resolution discovery and confirmation of copy number variants in 90 Yoruba Nigerians. Genome Biol 10:R125

Maubaret CG, Vaclavik V, Mukhopadhyay R, Waseem NH, Churchill A, Holder GE, Moore AT, Bhattacharya SS, Webster AR (2011) Autosomal dominant retinitis pigmentosa with intrafamilial variability and incomplete penetrance in two families carrying mutations in PRPF8. Invest Ophthalmol Vis Sci 52:9304-9309

Maugeri A, Flothmann K, Hemmrich N, Ingvast S, Jorge P, Paloma E, Patel R, Rozet JM, Tammur J, Testa F, Balcells S, Bird AC, Brunner HG, Hoyng CB, Metspalu A, Simonelli F, Allikmets R, Bhattacharya SS, D’Urso M, Gonzàlez-Duarte R, Kaplan J, te Meerman GJ, Santos R, Schwartz M, Van Camp G, Wadelius C, Weber BH, Cremers FP (2002) The ABCA4 2588G $>$ C Stargardt mutation: single origin and increasing frequency from SouthWest to North-East Europe. Eur J Hum Genet 10:197-203

Mavaddat N, Peock S, Frost D, Ellis S, Platte R, Fineberg E, Evans DG, Izatt L, Eeles RA, Adlard J, Davidson R, Eccles D, Cole T, Cook J, Brewer C, Tischkowitz M, Douglas F, Hodgson S, Walker L, Porteous ME, Morrison PJ, Side LE, Kennedy MJ, Houghton C, Donaldson A, Rogers MT, Dorkins H, Miedzybrodzka Z, Gregory H, Eason J, Barwell J, McCann E, Murray A, Antoniou AC, Easton DF, on behalf of EMBRACE (2013) Cancer risks for $B R C A 1$ and $B R C A 2$ mutation carriers: results from prospective analysis of EMBRACE. J Natl Cancer Inst 105:812-822

Mayeux R, Stern Y, Ottman R, Tatemichi TK, Tang MX, Maestre G, Ngai C, Tycko B, Ginsberg H (1993) The apolipoprotein epsilon 4 allele in patients with Alzheimer's disease. Ann Neurol 34:752-754

McColl M, Tait RC, Walker ID, Perry DJ, McCall F, Conkie JA (1996) Low thrombosis rate seen in blood donors and their relatives with inherited deficiencies of antithrombin and protein $\mathrm{C}$ : correlation with type of defect, family history, and absence of the factor $\mathrm{V}$ Leiden mutation. Blood Coagul Fibrinolysis 7:689-694

McCune CA, Ravine D, Carter K, Jackson HA, Hutton D, Hedderich J, Krawczak M, Worwood M (2006) Iron loading and morbidity 
among relatives of HFE C282Y homozygotes identified either by population genetic testing or presenting as patients. Gut 55:554-562

McDade E, Boeve BF, Burrus TM, Boot BP, Kantarci K, Fields J, Lowe VJ, Peller P, Knopman D, Baker M, Finch N, Rademakers R, Petersen R (2012) Similar clinical and neuroimaging features in monozygotic twin pair with mutation in progranulin. Neurology $78: 1245-1249$

McGee TL, Devoto M, Ott J, Berson EL, Dryja TP (1997) Evidence that the penetrance of mutations at the RP11 locus causing dominant retinitis pigmentosa is influenced by a gene linked to the homologous RP11 allele. Am J Hum Genet 61:1059-1066

McLaughlin JR, Risch HA, Lubinski J, Moller P, Ghadirian P, Lynch H, Karlan B, Fishman D, Rosen B, Neuhausen SL, Offit K, Kauff N, Domchek S, Tung N, Friedman E, Foulkes W, Sun P, Narod SA, Hereditary Ovarian Cancer Clinical Study Group (2007) Reproductive risk factors for ovarian cancer in carriers of $B R C A 1$ or $B R C A 2$ mutations: a case-control study. Lancet Oncol 8:26-34

McNeil SM, Novelletto A, Srinidhi J, Barnes G, Kornbluth I, Altherr MR, Wasmuth JJ, Gusella JF, MacDonald ME, Myers RH (1997) Reduced penetrance of the Huntington's disease mutation. Hum Mol Genet 6:775-779

Medeiros F, Lindor NM, Couch FJ, Highsmith WE Jr (2012) The germline $M L H 1$ K618A variant and susceptibility to Lynch syndrome-associated tumors. J Mol Diagn 14:264-273

Mégarbané A, Salem N, Stephan E, Ashoush R, Lenoir D, Delague V, Kassab R, Loiselet J, Bouvagnet P (2000) X-linked transposition of the great arteries and incomplete penetrance among males with a nonsense mutation in ZIC3. Eur J Hum Genet 8:704-708

Meggouh F, de Visser M, Arts WF, De Coo RI, van Schaik IN, Baas F (2005) Early onset neuropathy in a compound form of CharcotMarie-Tooth disease. Ann Neurol 57:589-591

Memisoglu A, Hu FB, Hankinson SE, Manson JE, De Vivo I, Willett WC, Hunter DJ (2003) Interaction between a peroxisome proliferator-activated receptor gamma gene polymorphism and dietary fat intake in relation to body mass. Hum Mol Genet 12:2923-2929

Mereuta OM, Baldovino S, Errichiello E, Binello GB, Restagno G, Battaglia GG, Mazzucco G, Roccatello D (2013) Systemic AA amyloidosis as a unique manifestation of a combined mutation of TNFRSF1A and MEFV genes. Amyloid 20:122-126

Merryweather-Clarke AT, Cadet E, Bomford A, Capron D, Viprakasit V, Miller A, McHugh PJ, Chapman RW, Pointon JJ, Wimhurst VL, Livesey KJ, Tanphaichitr V, Rochette J, Robson KJ (2003) Digenic inheritance of mutations in $H A M P$ and $H F E$ results in different types of haemochromatosis. Hum Mol Genet 12:2241-2247

Metzger S, Rong J, Nguyen HP, Cape A, Tomiuk J, Soehn AS, Propping P, Freudenberg-Hua Y, Freudenberg J, Tong L, Li SH, Li XJ, Riess O (2008) Huntingtin-associated protein-1 is a modifier of the age-at-onset of Huntington's disease. Hum Mol Genet 17:1137-1146

Meyle KD, Guldberg P (2009) Genetic risk factors for melanoma. Hum Genet 126:499-510

Michels M, Soliman OI, Phefferkorn J, Hoedemaekers YM, Kofflard MJ, Dooijes D, Majoor-Krakauer D, Ten Cate FJ (2009) Disease penetrance and risk stratification for sudden cardiac death in asymptomatic hypertrophic cardiomyopathy mutation carriers. Eur Heart J 30:2593-2598

Miesfeldt S, Turner BL, Lovell MA, Cooper MR, Lescallett J, Jones SM (1998) A novel BRCAl mutation in an identical twin pair with similar clinical histories. Cancer Genet Cytogenet 100:43-48

Migita K, Agematsu K, Masumoto J, Ida H, Honda S, Jiuchi Y, Izumi Y, Maeda Y, Uehara R, Nakamura Y, Koga T, Kawakami A,
Nakashima M, Fujeida Y, Nonaka F, Eguchi K, Furukawa H, Nakamura T, Nakamura M, Yasunami M (2013) The contribution of $S A A 1$ polymorphisms to familial Mediterranean fever susceptibility in the Japanese population. PLoS ONE 8:e55227

Milanesi E, Bonvicini C, Alberici A, Pilotto A, Cattane N, Premi E, Gazzina S, Archetti S, Gasparotti R, Cancelli V, Gennarelli M, Padovani A, Borroni B (2013) Molecular signature of disease onset in granulin mutation carriers: a gene expression analysis study. Neurobiol Aging 34:1837-1845

Milet J, Dehais V, Bourgain C, Jouanolle AM, Mosser A, Perrin M, Morcet J, Brissot P, David V, Deugnier Y, Mosser J (2007) Common variants in the $B M P 2, B M P 4$, and $H J V$ genes of the hepcidin regulation pathway modulate $H F E$ hemochromatosis penetrance. Am J Hum Genet 81:799-807

Millar DS, Kemball-Cook G, McVey JH, Tuddenham EG, Mumford AD, Attock GB, Reverter JC, Lanir N, Parapia LA, Reynaud J, Meili E, von Felton A, Martinowitz U, Prangnell DR, Krawczak M, Cooper DN (2000) Molecular analysis of the genotypephenotype relationship in factor VII deficiency. Hum Genet 107:327-342

Millat G, Bouvagnet P, Chevalier P, Dauphin C, Jouk PS, Da Costa A, Prieur F, Bresson JL, Faivre L, Eicher JC, Chassaing N, Crehalet H, Porcher R, Rodriguez-Lafrasse C, Rousson R (2010) Prevalence and spectrum of mutations in a cohort of 192 unrelated patients with hypertrophic cardiomyopathy. Eur J Med Genet 53:261-267

Milos IN, Frank-Raue K, Wohllk N, Maia AL, Pusiol E, Patocs A, Robledo M, Biarnes J, Barontini M, Links TP, de Groot JW, Dvorakova S, Peczkowska M, Rybicki LA, Sullivan M, Raue F, Zosin I, Eng C, Neumann HP (2008) Age-related neoplastic risk profiles and penetrance estimations in multiple endocrine neoplasia type 2A caused by germ line RET Cys634Trp (TGC > TGG) mutation. Endocr Relat Cancer 15:1035-1041

Mitne-Neto M, Kok F, Beetz C, Pessoa A, Bueno C, Graciani Z, Martyn M, Monteiro CB, Mitne G, Hubert P, Nygren AO, Valadares M, Cerqueira AM, Starling A, Deufel T, Zatz M (2007) A multi-exonic SPG4 duplication underlies sex-dependent penetrance of hereditary spastic paraplegia in a large Brazilian pedigree. Eur J Hum Genet 15:1276-1279

Mondal K, Ramachandran D, Patel VC, Hagen KR, Bose P, Cutler DJ, Zwick ME (2012) Excess variants in $A F F 2$ detected by massively parallel sequencing of males with autism spectrum disorder. Hum Mol Genet 21:4356-4364

Morak M, Massdorf T, Sykora H, Kerscher M, Holinski-Feder E (2011) First evidence for digenic inheritance in hereditary colorectal cancer by mutations in the base excision repair genes. Eur J Cancer 47:1046-1055

Morell R, Spritz RA, Ho L, Pierpont J, Guo W, Friedman TB, Asher JH Jr (1997) Apparent digenic inheritance of Waardenburg syndrome type 2 (WS2) and autosomal recessive ocular albinism (AROA). Hum Mol Genet 6:659-664

Morgan NV, Hartley JL, Setchell KD, Simpson MA, Brown R, Tee L, Kirkham S, Pasha S, Trembath RC, Maher ER, Gissen P, Kelly DA (2013) A combination of mutations in AKR1D1 and SKIV2L in a family with severe infantile liver disease. Orphanet J Rare Dis 8:74

Müller B, Hedrich K, Kock N, Dragasevic N, Svetel M, Garrels J, Landt O, Nitschke M, Pramstaller PP, Reik W, Schwinger E, Sperner J, Ozelius L, Kostic V, Klein C (2002) Evidence that paternal expression of the epsilon-sarcoglycan gene accounts for reduced penetrance in myoclonus-dystonia. Am J Hum Genet 71:1303-1311

Mulley JC, Scheffer IE, Desai T, Bayly MA, Grinton BE, Vears DF, Berkovic SF, Dibbens LM (2011) Investigation of the 15q13.3 $\mathrm{CNV}$ as a genetic modifier for familial epilepsies with variable phenotypes. Epilepsia 52:e139-e142 
Munhoz RP, Wakutani Y, Marras C, Teive HA, Raskin S, Werneck LC, Moreno D, Sato C, Lang AE, Rogaeva E (2008) The G2019S LRRK2 mutation in Brazilian patients with Parkinson's disease: phenotype in monozygotic twins. Mov Disord 23:290-294

Muntoni F, Bonne G, Goldfarb LG, Mercuri E, Piercy RJ, Burke M, Yaou RB, Richard P, Récan D, Shatunov A, Sewry CA, Brown SC (2006) Disease severity in dominant Emery Dreifuss is increased by mutations in both emerin and desmin proteins. Brain 129:1260-1268

Nadeau JH (2003) Modifier genes and protective alleles in humans and mice. Curr Opin Genet Dev 13:290-295

Nahhas F, Garbern J, Feely S, Feldman GL (2009) An intergenerational contraction of a fully penetrant Huntington disease allele to a reduced penetrance allele: interpretation of results and significance for risk assessment and genetic counseling. Am J Med Genet 149A:732-736

Nakajima T, Kaneko Y, Irie T, Takahashi R, Kato T, Iijima T, Iso T, Kurabayashi M (2012) Compound and digenic heterozygosity in desmosome genes as a cause of arrhythmogenic right ventricular cardiomyopathy in Japanese patients. Circ J 76:737-743

Neale BM, Kou Y, Liu L, Ma'ayan A, Samocha KE, Sabo A, Lin CF, Stevens C, Wang LS, Makarov V, Polak P, Yoon S, Maguire J, Crawford EL, Campbell NG, Geller ET, Valladares O, Schafer C, Liu H, Zhao T, Cai G, Lihm J, Dannenfelser R, Jabado O, Peralta Z, Nagaswamy U, Muzny D, Reid JG, Newsham I, Wu Y, Lewis L, Han Y, Voight BF, Lim E, Rossin E, Kirby A, Flannick J, Fromer M, Shakir K, Fennell T, Garimella K, Banks E, Poplin R, Gabriel S, DePristo M, Wimbish JR, Boone BE, Levy SE, Betancur C, Sunyaev S, Boerwinkle E, Buxbaum JD, Cook EH Jr, Devlin B, Gibbs RA, Roeder K, Schellenberg GD, Sutcliffe JS, Daly MJ (2012) Patterns and rates of exonic de novo mutations in autism spectrum disorders. Nature 485:242-245

Nelson MR, Wegmann D, Ehm MG, Kessner D, St Jean P, Verzilli C, Shen J, Tang Z, Bacanu SA, Fraser D, Warren L, Aponte J, Zawistowski M, Liu X, Zhang H, Zhang Y, Li J, Li Y, Li L, Woollard P, Topp S, Hall MD, Nangle K, Wang J, Abecasis G, Cardon LR, Zöllner S, Whittaker JC, Chissoe SL, Novembre J, Mooser V (2012) An abundance of rare functional variants in 202 drug target genes sequenced in 14,002 people. Science 337:100-104

Ng PC, Levy S, Huang J, Stockwell TB, Walenz BP, Li K, Axelrod N, Busam DA, Strausberg RL, Venter JC (2008) Genetic variation in an individual human exome. PLoS Genet 4:e1000160

Niksic M, Romano M, Buratti E, Pagani F, Baralle FE (1999) Functional analysis of cis-acting elements regulating the alternative splicing of human CFTR exon 9. Hum Mol Genet 8:2339-2349

Nishiguchi KM, Rivolta C (2012) Genes associated with retinitis pigmentosa and allied diseases are frequently mutated in the general population. PLoS ONE 7:e41902

Nishio Y, Makiyama T, Itoh H, Sakaguchi T, Ohno S, Gong YZ, Yamamoto S, Ozawa T, Ding WG, Toyoda F, Kawamura M, Akao M, Matsuura H, Kimura T, Kita T, Horie M (2009) D85N, a $K C N E 1$ polymorphism, is a disease-causing gene variant in long QT syndrome. J Am Coll Cardiol 54:812-819

Nissim-Rafinia M, Kerem B (2005) The splicing machinery is a genetic modifier of disease severity. Trends Genet 21:480-483

Niu DM, Hwang B, Hwang HW, Wang NH, Wu JY, Lee PC, Chien JC, Shieh RC, Chen YT (2006) A common SCN5A polymorphism attenuates a severe cardiac phenotype caused by a nonsense SCN5A mutation in a Chinese family with an inherited cardiac conduction defect. J Med Genet 43:817-821

Noguchi T, Katsuda S, Kawashiri MA, Tada H, Nohara A, Inazu A, Yamagishi M, Kobayashi J, Mabuchi H (2010) The E32K variant of $P C S K 9$ exacerbates the phenotype of familial hypercholesterolaemia by increasing PCSK 9 function and concentration in the circulation. Atherosclerosis 210:166-172

Nolte D, Sobanski E, Wissen A, Regula JU, Lichy C, Müller U (2010) Spinocerebellar ataxia type 17 associated with an expansion of 42 glutamine residues in TATA-box binding protein gene. J Neurol Neurosurg Psychiatry 81:1396-1399

Nossent AY, VAN Marion V, VAN Tilburg NH, Rosendaal FR, Bertina RM, VAN Mourik JA, Eikenboom HC (2006) von Willebrand factor and its propeptide: the influence of secretion and clearance on protein levels and the risk of venous thrombosis. J Thromb Haemost 4:2556-2562

Nozu K, Inagaki T, Fu XJ, Nozu Y, Kaito H, Kanda K, Sekine T, Igarashi T, Nakanishi K, Yoshikawa N, Iijima K, Matsuo M (2008) Molecular analysis of digenic inheritance in Bartter syndrome with sensorineural deafness. J Med Genet 45:182-186

Núñez L, Barana A, Amorós I, de la Fuente MG, Dolz-Gaitón P, Gómez R, Rodríguez-García I, Mosquera I, Monserrat L, Delpón E, Caballero R, Castro-Beiras A, Tamargo J (2013) p.D1690N Nav1.5 rescues p.G1748D mutation gating defects in a compound heterozygous Brugada syndrome patient. Heart Rhythm $10: 264-272$

Núñez-Torres R, Fernández RM, Acosta MJ, Enguix-Riego Mdel V, Marbá M, Carlos de Agustín J, Castaño L, Antiñolo G, Borrego $\mathrm{S}$ (2011) Comprehensive analysis of RET common and rare variants in a series of Spanish Hirschsprung patients confirms a synergistic effect of both kinds of events. BMC Med Genet $12: 138$

Nuytemans K, Bademci G, Inchausti V, Dressen A, Kinnamon DD, Mehta A, Wang L, Züchner S, Beecham GW, Martin ER, Scott WK, Vance JM (2013) Whole exome sequencing of rare variants in EIF4G1 and VPS35 in Parkinson disease. Neurology 80:982-989

O'Gorman C, Lucas R, Taylor B (2012) Environmental risk factors for multiple sclerosis: a review with a focus on molecular mechanisms. Int J Mol Sci 13:11718-11752

Ober C, Loisel DA, Gilad Y (2008) Sex-specific genetic architecture of human disease. Nat Rev Genet 9:911-922

Oda M, Maruyama $\mathrm{H}$, Komure $\mathrm{O}$, Morino $\mathrm{H}$, Terasawa $\mathrm{H}$, Izumi $\mathrm{Y}$, Imamura T, Yasuda M, Ichikawa K, Ogawa M, Matsumoto M, Kawakami H (2004) Possible reduced penetrance of expansion of 44 to $47 \mathrm{CAG} / \mathrm{CAA}$ repeats in the TATA-binding protein gene in spinocerebellar ataxia type 17. Arch Neurol 61:209-212

Ogaki K, Li Y, Atsuta N, Tomiyama H, Funayama M, Watanabe H, Nakamura R, Yoshino H, Yato S, Tamura A, Naito Y, Taniguchi A, Fujita K, Izumi Y, Kaji R, Hattori N, Sobue G, Japanese Consortium for Amyotrophic Lateral Sclerosis research (JaCALS) (2012) Analysis of C9orf72 repeat expansion in 563 Japanese patients with amyotrophic lateral sclerosis. Neurobiol Aging 33:2527

Ohmori I, Ouchida M, Miki T, Mimaki N, Kiyonaka S, Nishiki T, Tomizawa K, Mori Y, Matsui H (2008) A CACNB4 mutation shows that altered $\mathrm{Ca}_{\mathrm{v}} 2.1$ function may be a genetic modifier of severe myoclonic epilepsy in infancy. Neurobiol Dis 32:349-354

Ohmori I, Ouchida M, Kobayashi K, Jitsumori Y, Mori A, Michiue H, Nishiki T, Ohtsuka Y, Matsui H (2013) CACNA1A variants may modify the epileptic phenotype of Dravet syndrome. Neurobiol Dis 50:209-217

Okkels H, Sunde L, Lindorff-Larsen K, Thorlacius-Ussing O, Gandrup P, Lindebjerg J, Stubbeteglbjaerg P, Oestergaard JR, Nielsen FC, Krarup HB (2006) Polyposis and early cancer in a patient with low penetrant mutations in MSH6 and $A P C$ : hereditary colorectal cancer as a polygenic trait. Int J Colorectal Dis $21: 847-850$

Oliva-Sandoval MJ, Ruiz-Espejo F, Monserrat L, Hermida-Prieto M, Sabater M, García-Molina E, Ortiz M, Rodríguez-García MI, 
Núñez L, Gimeno JR, Castro-Beiras A, Valdés M (2010) Insights into genotype-phenotype correlation in hypertrophic cardiomyopathy. Findings from 18 Spanish families with a single mutation in MYBPC3. Heart 96:1980-1984

Onadim Z, Hogg A, Baird PN, Cowell JK (1992) Oncogenic point mutations in exon 20 of the $R B 1$ gene in families showing incomplete penetrance and mild expression of the retinoblastoma phenotype. Proc Natl Acad Sci USA 89:6177-6181

Oprea GE, Kröber S, McWhorter ML, Rossoll W, Müller S, Krawczak M, Bassell GJ, Beattie CE, Wirth B (2008) Plastin 3 is a protective modifier of autosomal recessive spinal muscular atrophy. Science 320:524-527

Ostrer H, Skorecki K (2013) The population genetics of the Jewish people. Hum Genet 132:119-127

Otterson GA, Wd Chen, Coxon AB, Khleif SN, Kaye FJ (1997) Incomplete penetrance of familial retinoblastoma linked to germ-line mutations that result in partial loss of RB function. Proc Natl Acad Sci USA 94:12036-12040

Otterson GA, Modi S, Nguyen K, Coxon AB, Kaye FJ (1999) Temperature-sensitive RB mutations linked to incomplete penetrance of familial retinoblastoma in 12 families. Am J Hum Genet 65:1040-1046

Otto PA, Maestrelli SR (2000) Heterozygosity probabilities for normal relatives of isolated cases affected by incompletely penetrant conditions and the calculation of recurrence risks for their offspring. I. Autosomal dominant genes. Am J Med Genet 95:43-48

Paavonen KJ, Swan H, Piippo K, Laitinen P, Fodstad H, Sarna S, Toivonen L, Kontula K, Viitasalo M (2007) Beta1-adrenergic receptor polymorphisms, QTc interval and occurrence of symptoms in type 1 of long QT syndrome. Int J Cardiol 118:197-202

Padalon-Brauch G, Amitai DB, Vodo D, Harel A, Sarig O, Sprecher E, Mashiah J (2012) Digenic inheritance in epidermolysis bullosa simplex. J Invest Dermatol 132:2852-2854

Page SP, Kounas S, Syrris P, Christiansen M, Frank-Hansen R, Andersen PS, Elliott PM, McKenna WJ (2012) Cardiac myosin binding protein-C mutations in families with hypertrophic cardiomyopathy: disease expression in relation to age, gender, and long term outcome. Circ Cardiovasc Genet 5:156-166

Palacios R, Gazave E, Goñi J, Piedrafita G, Fernando O, Navarro A, Villoslada P (2009) Allele-specific gene expression is widespread across the genome and biological processes. PLoS ONE 4:e4150

Panegyres PK, Goh JG (2011) The neurology and natural history of patients with indeterminate CAG repeat length mutations of the Huntington disease gene. J Neurol Sci 301:14-20

Pantakani DV, Zechner U, Arygriou L, Pauli S, Sauter SM, Mannan AU (2008) Compound heterozygosity in the SPG4 gene causes hereditary spastic paraplegia. Clin Genet 73:268-272

Park Y, Kubo A, Komiya T, Coxon A, Beebe K, Neckers L, Meltzer PS, Kaye FJ (2008) Low-penetrant RB allele in small-cell cancer shows geldanamycin instability and discordant expression with mutant ras. Cell Cycle 7:2384-2391

Pedroni M, Di Gregorio C, Cortesi L, Reggiani Bonetti L, Magnani G, Simone ML, Medici V, Priore Oliva C, Marino M, Ponz de Leon M (2013) Double heterozygosity for BRCA1 and $h M L H 1$ gene mutations in a 46-year-old woman with five primary tumors. Tech Coloproctol (in press)

Pei Y, Paterson AD, Wang KR, He N, Hefferton D, Watnick T, Germino GG, Parfrey P, Somlo S, St George-Hyslop P (2001) Bilineal disease and trans-heterozygotes in autosomal dominant polycystic kidney disease. Am J Hum Genet 68:355-363

Pelucchi S, Mariani R, Calza S, Fracanzani AL, Modignani GL, Bertola F, Busti F, Trombini P, Fraquelli M, Forni GL, Girelli D, Fargion S, Specchia C, Piperno A (2012) CYBRD1 as a modifier gene that modulates iron phenotype in HFE p.C282Y homozygous patients. Haematologica 97:1818-1825
Pereira TV, Mingroni-Netto RC, Yamada Y (2011) ADRB2 and LEPR gene polymorphisms: synergistic effects on the risk of obesity in Japanese. Obesity 19:1523-1527

Pern F, Bogdanova N, Schürmann P, Lin M, Ay A, Länger F, Hillemanns P, Christiansen H, Park-Simon TW, Dörk T (2012) Mutation analysis of BRCA1, BRCA2, PALB2 and BRD7 in a hospital-based series of German patients with triple-negative breast cancer. PLoS ONE 7:e47993

Phillips JA 3rd, Poling JS, Phillips CA, Stanton KC, Austin ED, Cogan JD, Wheeler L, Yu C, Newman JH, Dietz HC, Loyd JE (2008) Synergistic heterozygosity for TGFbeta1 SNPs and BMPR2 mutations modulates the age at diagnosis and penetrance of familial pulmonary arterial hypertension. Genet Med 10:359-365

Pinto D, Pagnamenta AT, Klei L, Anney R, Merico D, Regan R, Conroy J, Magalhaes TR, Correia C, Abrahams BS, Almeida J, Bacchelli E, Bader GD, Bailey AJ, Baird G, Battaglia A, Berney T, Bolshakova N, Bölte S, Bolton PF, Bourgeron T, Brennan S, Brian J, Bryson SE, Carson AR, Casallo G, Casey J, Chung BH, Cochrane L, Corsello C, Crawford EL, Crossett A, Cytrynbaum C, Dawson G, de Jonge M, Delorme R, Drmic I, Duketis E, Duque F, Estes A, Farrar P, Fernandez BA, Folstein SE, Fombonne E, Freitag CM, Gilbert J, Gillberg C, Glessner JT, Goldberg J, Green A, Green J, Guter SJ, Hakonarson H, Heron EA, Hill M, Holt R, Howe JL, Hughes G, Hus V, Igliozzi R, Kim C, Klauck SM, Kolevzon A, Korvatska O, Kustanovich V, Lajonchere CM, Lamb JA, Laskawiec M, Leboyer M, Le Couteur A, Leventhal BL, Lionel AC, Liu XQ, Lord C, Lotspeich L, Lund SC, Maestrini E, Mahoney W, Mantoulan C, Marshall CR, McConachie H, McDougle CJ, McGrath J, McMahon WM, Merikangas A, Migita O, Minshew NJ, Mirza GK, Munson J, Nelson SF, Noakes C, Noor A, Nygren G, Oliveira G, Papanikolaou K, Parr JR, Parrini B, Paton T, Pickles A, Pilorge M, Piven J, Ponting CP, Posey DJ, Poustka A, Poustka F, Prasad A, Ragoussis J, Renshaw K, Rickaby J, Roberts W, Roeder K, Roge B, Rutter ML, Bierut LJ, Rice JP, Salt J, Sansom K, Sato D, Segurado R, Sequeira AF, Senman L, Shah N, Sheffield VC, Soorya L, Sousa I, Stein O, Sykes N, Stoppioni V, Strawbridge C, Tancredi R, Tansey K, Thiruvahindrapduram B, Thompson AP, Thomson S, Tryfon A, Tsiantis J, Van Engeland H, Vincent JB, Volkmar F, Wallace S, Wang K, Wang Z, Wassink TH, Webber C, Weksberg R, Wing $\mathrm{K}$, Wittemeyer K, Wood S, Wu J, Yaspan BL, Zurawiecki D, Zwaigenbaum L, Buxbaum JD, Cantor RM, Cook EH, Coon H, Cuccaro ML, Devlin B, Ennis S, Gallagher L, Geschwind DH, Gill M, Haines JL, Hallmayer J, Miller J, Monaco AP, Nurnberger JI Jr, Paterson AD, Pericak-Vance MA, Schellenberg GD, Szatmari P, Vicente AM, Vieland VJ, Wijsman EM, Scherer SW, Sutcliffe JS, Betancur C (2010) Functional impact of global rare copy number variation in autism spectrum disorders. Nature 466:368-372

Pisciotta L, Priore Oliva C, Cefalù AB, Noto D, Bellocchio A, Fresa R, Cantafora A, Patel D, Averna M, Tarugi P, Calandra S, Bertolini S (2006) Additive effect of mutations in $L D L R$ and PCSK 9 genes on the phenotype of familial hypercholesterolemia. Atherosclerosis 186:433-440

Pitteloud N, Quinton R, Pearce S, Raivio T, Acierno J, Dwyer A, Plummer L, Hughes V, Seminara S, Cheng YZ, Li WP, Maccoll G, Eliseenkova AV, Olsen SK, Ibrahimi OA, Hayes FJ, Boepple P, Hall JE, Bouloux P, Mohammadi M, Crowley W (2007) Digenic mutations account for variable phenotypes in idiopathic hypogonadotropic hypogonadism. J Clin Invest 117:457-463

Plassart-Schiess E, Gervais A, Eymard B, Lagueny A, Pouget J, Warter JM, Fardeau M, Jentsch TJ, Fontaine B (1998) Novel muscle chloride channel (CLCN1) mutations in myotonia congenita with various modes of inheritance including incomplete dominance and penetrance. Neurology 50:1176-1179 
Plenge RM, Tranebjaerg L, Jensen PK, Schwartz C, Willard HF (1999) Evidence that mutations in the X-linked DDP gene cause incompletely penetrant and variable skewed $\mathrm{X}$ inactivation. Am J Hum Genet 64:759-767

Plon SE, Eccles DM, Easton D, Foulkes WD, Genuardi M, Greenblatt MS, Hogervorst FB, Hoogerbrugge N, Spurdle AB, Tavtigian SV, IARC Unclassified Genetic Variants Working Group (2008) Sequence variant classification and reporting: recommendations for improving the interpretation of cancer susceptibility genetic test results. Hum Mutat 29:1282-1291

Plon SE, Wheeler DA, Strong LC, Tomlinson GE, Pirics M, Meng Q, Cheung HC, Begin PR, Muzny DM, Lewis L, Biegel JA, Gibbs RA (2011) Identification of genetic susceptibility to childhood cancer through analysis of genes in parallel. Cancer Genet 204:19-25

Podder S, Ghosh TC (2011) Insights into the molecular correlates modulating functional compensation between monogenic and polygenic disease gene duplicates in human. Genomics 97:200-204

Preda L, Dinca O, Bucur A, Dragomir C, Severin E (2010) Identical mutation in $S H 3 B P 2$ gene causes clinical phenotypes with different severity in mother and daughter-case report. Mol Syndromol 1:87-90

Prior TW, Krainer AR, Hua Y, Swoboda KJ, Snyder PC, Bridgeman SJ, Burghes AH, Kissel JT (2009) A positive modifier of spinal muscular atrophy in the SMN2 gene. Am J Hum Genet 85:408-413

Qiu LX, Wang Y, Xia ZG, Xi B, Mao C, Wang JL, Wang BY, Lv FF, Wu XH, Hu LQ (2011) miR-196a2 C allele is a low-penetrant risk factor for cancer development. Cytokine 56:589-592

Quarrell OW, Rigby AS, Barron L, Crow Y, Dalton A, Dennis N, Fryer AE, Heydon F, Kinning E, Lashwood A, Losekoot M, Margerison L, McDonnell S, Morrison PJ, Norman A, Peterson M, Raymond FL, Simpson S, Thompson E, Warner J (2007) Reduced penetrance alleles for Huntington's disease: a multicentre direct observational study. J Med Genet 44:e68

Quaynor SD, Kim HG, Cappello EM, Williams T, Chorich LP, Bick DP, Sherins RJ, Layman LC (2011) The prevalence of digenic mutations in patients with normosmic hypogonadotropic hypogonadism and Kallmann syndrome. Fertil Steril 96:1424-1430

Raef H, Baitei EY, Zou M, Shi Y (2008) Genotype-phenotype correlation in a family with primary cortisol resistance: possible modulating effect of the ER22/23EK polymorphism. Eur $\mathrm{J}$ Endocrinol 158:577-582

Raivio T, Sidis Y, Plummer L, Chen H, Ma J, Mukherjee A, Jacobson-Dickman E, Quinton R, Van Vliet G, Lavoie H, Hughes VA, Dwyer A, Hayes FJ, Xu S, Sparks S, Kaiser UB, Mohammadi M, Pitteloud N (2009) Impaired fibroblast growth factor receptor 1 signaling as a cause of normosmic idiopathic hypogonadotropic hypogonadism. J Clin Endocrinol Metab 94:4380-4390

Ramachandrappa S, Farooqi IS (2011) Genetic approaches to understanding human obesity. J Clin Invest 121:2080-2086

Rana HQ, Balwani M, Bier L, Alcalay RN (2013) Age-specific Parkinson disease risk in $G B A$ mutation carriers: information for genetic counseling. Genet Med 15:146-149

Rankin J, Auer-Grumbach M, Bagg W, Colclough K, Nguyen TD, Fenton-May J, Hattersley A, Hudson J, Jardine P, Josifova D, Longman C, McWilliam R, Owen K, Walker M, Wehnert M, Ellard S (2008) Extreme phenotypic diversity and nonpenetrance in families with the LMNA gene mutation R644C. Am J Med Genet 146A:1530-1542

Rasmussen TB, Palmfeldt J, Nissen PH, Magnoni R, Dalager S, Jensen UB, Kim WY, Heickendorff L, Mølgaard H, Jensen HK, Baandrup UT, Bross P, Mogensen J (2013) Mutated desmoglein2 proteins are incorporated into desmosomes and exhibit dominant-negative effects in arrhythmogenic right ventricular cardiomyopathy. Hum Mutat 34:697-705

Rave-Harel N, Kerem E, Nissim-Rafinia M, Madjar I, Goshen R, Augarten A, Rahat A, Hurwitz A, Darvasi A, Kerem B (1997) The molecular basis of partial penetrance of splicing mutations in cystic fibrosis. Am J Hum Genet 60:87-94

Refsgaard L, Holst AG, Sadjadieh G, Haunsø S, Nielsen JB, Olesen MS (2012) High prevalence of genetic variants previously associated with LQT syndrome in new exome data. Eur J Hum Genet 20:905-908

Richard P, Charron P, Carrier L, Ledeuil C, Cheav T, Pichereau C, Benaiche A, Isnard R, Dubourg O, Burban M, Gueffet JP, Millaire A, Desnos M, Schwartz K, Hainque B, Komajda M, EUROGENE Heart Failure Project (2003) Hypertrophic cardiomyopathy: distribution of disease genes, spectrum of mutations, and implications for a molecular diagnosis strategy. Circulation 107:2227-2232

Rigaud S, Lopez-Granados E, Sibéril S, Gloire G, Lambert N, Lenoir C, Synaeve C, Stacey M, Fugger L, Stephan JL, Fischer A, Picard C, Durandy A, Chapel H, Latour S (2011) Human $\mathrm{X}$-linked variable immunodeficiency caused by a hypomorphic mutation in XIAP in association with a rare polymorphism in CD4OLG. Blood 118:252-261

Rio Frio T, Civic N, Ransijn A, Beckmann JS, Rivolta C (2008) Two trans-acting eQTLs modulate the penetrance of PRPF31 mutations. Hum Mol Genet 17:3154-3165

Rio Frio T, McGee TL, Wade NM, Iseli C, Beckmann JS, Berson EL, Rivolta C (2009) A single-base substitution within an intronic repetitive element causes dominant retinitis pigmentosa with reduced penetrance. Hum Mutat 30:1340-1347

Risch HA, McLaughlin JR, Cole DE, Rosen B, Bradley L, Kwan E, Jack E, Vesprini DJ, Kuperstein G, Abrahamson JL, Fan I, Wong B, Narod SA (2001) Prevalence and penetrance of germline BRCA1 and BRCA2 mutations in a population series of 649 women with ovarian cancer. Am J Hum Genet 68:700-710

Risch NJ, Bressman SB, Senthil G, Ozelius LJ (2007) Intragenic cis and trans modification of genetic susceptibility in DYT1 torsion dystonia. Am J Hum Genet 80:1188-1193

Rivolta C, McGee TL, Rio Frio T, Jensen RV, Berson EL, Dryja TP (2006) Variation in retinitis pigmentosa-11 (PRPF31 or RP11) gene expression between symptomatic and asymptomatic patients with dominant RP11 mutations. Hum Mutat 27:644-653

Robson KJ, Lehmann DJ, Wimhurst VL, Livesey KJ, Combrinck M, Merryweather-Clarke AT, Warden DR, Smith AD (2004) Synergy between the $\mathrm{C} 2$ allele of transferrin and the $\mathrm{C} 282 \mathrm{Y}$ allele of the haemochromatosis gene (HFE) as risk factors for developing Alzheimer's disease. J Med Genet 41:261-265

Rochette J, Le Gac G, Lassoued K, Férec C, Robson KJ (2010) Factors influencing disease phenotype and penetrance in $H F E$ haemochromatosis. Hum Genet 128:233-248

Rodeghiero F, Castaman G, Dini E (1987) Epidemiological investigation of the prevalence of von Willebrand's disease. Blood 69:454-459

Rodriguez-Antona C, Gomez A, Karlgren M, Sim SC, IngelmanSundberg M (2010) Molecular genetics and epigenetics of the cytochrome $\mathrm{P} 450$ gene family and its relevance for cancer risk and treatment. Hum Genet 127:1-17

Rohlfs EM, Zhou Z, Heim RA, Nagan N, Rosenblum LS, Flynn K, Scholl T, Akmaev VR, Sirko-Osadsa DA, Allitto BA, Sugarman EA (2011) Cystic fibrosis carrier testing in an ethnically diverse US population. Clin Chem 57:841-848

Roncarati R, Viviani Anselmi C, Krawitz P, Lattanzi G, von Kodolitsch Y, Perrot A, di Pasquale E, Papa L, Portararo P, Columbaro M, Forni A, Faggian G, Condorelli G, Robinson PN (2013) Doubly heterozygous $L M N A$ and $T T N$ mutations revealed 
by exome sequencing in a severe form of dilated cardiomyopathy. Eur J Hum Genet (in press)

Rosendahl J, Landt O, Bernadova J, Kovacs P, Teich N, Bodeker H, Keim V, Ruffert C, Mossner J, Kage A, Stumvoll M, Groneberg D, Kruger R, Luck W, Treiber M, Becker M, Witt H (2013) CFTR, SPINK1, CTRC and PRSS1 variants in chronic pancreatitis: is the role of mutated CFTR overestimated? Gut 62:582-592

Rosenfeld JA, Coe BP, Eichler EE, Cuckle H, Shaffer LG (2013) Estimates of penetrance for recurrent pathogenic copy-number variations. Genet Med 15:478-481

Rossetti S, Kubly VJ, Consugar MB, Hopp K, Roy S, Horsley SW, Chauveau D, Rees L, Barratt TM, van't Hoff WG, Niaudet P, Torres VE, Harris PC (2009) Incompletely penetrant PKDI alleles suggest a role for gene dosage in cyst initiation in polycystic kidney disease. Kidney Int 75:848-855

Rossi E, Olynyk JK, Jeffrey GP (2008) Clinical penetrance of C282Y homozygous HFE hemochromatosis. Expert Rev Hematol $1: 205-216$

Roumenina LT, Frimat M, Miller EC, Provot F, Dragon-Durey MA, Bordereau P, Bigot S, Hue C, Satchell SC, Mathieson PW, Mousson C, Noel C, Sautes-Fridman C, Halbwachs-Mecarelli L, Atkinson JP, Lionet A, Fremeaux-Bacchi V (2012) A prevalent C3 mutation in aHUS patients causes a direct $\mathrm{C} 3$ convertase gain of function. Blood 119:4182-4191

Roxburgh RH, Marquis-Nicholson R, Ashton F, George AM, Lea RA, Eccles D, Mossman S, Bird T, van Gassen KL, Kamsteeg EJ, Love DR (2013) The p.Ala510Val mutation in the SPG7 (paraplegin) gene is the most common mutation causing adult onset neurogenetic disease in patients of British ancestry. J Neurol 260:1286-1294

Ruiz-Martínez J, Gorostidi A, Ibañez B, Alzualde A, Otaegui D, Moreno F, López de Munain A, Bergareche A, Gómez-Esteban JC, Martí Massó JF (2010) Penetrance in Parkinson's disease related to the LRRK2 R1441G mutation in the Basque country (Spain). Mov Disord 25:2340-2345

Runyon RS, Cachola LM, Rajeshuni N, Hunter T, Garcia M, Ahn R, Lurmann F, Krasnow R, Jack LM, Miller RL, Swan GE, Kohli A, Jacobson AC, Nadeau KC (2012) Asthma discordance in twins is linked to epigenetic modifications of T cells. PLoS ONE 7:e48796

Ryan JG, Masters SL, Booty MG, Habal N, Alexander JD, Barham BK, Remmers EF, Barron KS, Kastner DL, Aksentijevich I (2010) Clinical features and functional significance of the P369S/R408Q variant in pyrin, the familial Mediterranean fever protein. Ann Rheum Dis 69:1383-1388

Sagong B, Baek J-I, Oh S-K, Na KJ, Bae JW, Choi SY, Jeong JY, Choi JY, Lee S-H, Lee K-Y, Kim U-K (2013) A rapid method for simultaneous screening of multi-gene mutations associated with hearing loss in the Korean population. PLoS ONE 8:e57237

Saini S, Robinson PN, Singh JR, Vanita V (2012) A novel 7 bp deletion in PRPF31 associated with autosomal dominant retinitis pigmentosa with incomplete penetrance in an Indian family. Exp Eye Res 104:82-88

Sampieri K, Hadjistilianou T, Mari F, Speciale C, Mencarelli MA, Cetta F, Manoukian S, Peissel B, Giachino D, Pasini B, Acquaviva A, Caporossi A, Frezzotti R, Renieri A, Bruttini M (2006) Mutational screening of the $R B 1$ gene in Italian patients with retinoblastoma reveals 11 novel mutations. J Hum Genet 51:209-216

San Millán JL, Botella-Carretero JI, Alvarez-Blasco F, LuqueRamírez M, Sancho J, Moghetti P, Escobar-Morreale HF (2005) A study of the hexose-6-phosphate dehydrogenase gene R453Q and 11beta-hydroxysteroid dehydrogenase type 1 gene 83557insA polymorphisms in the polycystic ovary syndrome. J Clin Endocrinol Metab 90:4157-4162
Sánchez-Mejías A, Fernández RM, López-Alonso M, Antiñolo G, Borrego S (2009) Contribution of RET, NTRK3 and EDN3 to the expression of Hirschsprung disease in a multiplex family. J Med Genet 46:862-864

Sánchez-Sánchez F, Kruetzfeldt M, Nájera C, Mittnacht S (2005) A novel constitutional mutation affecting splicing of retinoblastoma tumor suppressor gene intron 23 causes partial loss of pRB activity. Hum Mutat 25:223

Sánchez-Sánchez F, Ramírez-Castillejo C, Weekes DB, Beneyto M, Prieto F, Nájera C, Mittnacht S (2007) Attenuation of disease phenotype through alternative translation initiation in lowpenetrance retinoblastoma. Hum Mutat 28:159-167

Sando SB, Melquist S, Cannon A, Hutton ML, Sletvold O, Saltvedt I, White LR, Lydersen S, Aasly JO (2008) APOE epsilon 4 lowers age at onset and is a high risk factor for Alzheimer's disease; a case control study from central Norway. BMC Neurol 8:9

Santana A, Salido E, Torres A, Shapiro LJ (2003) Primary hyperoxaluria type 1 in the Canary Islands: a conformational disease due to I244T mutation in the P11L-containing alanine:glyoxylate aminotransferase. Proc Natl Acad Sci USA 100:7277-7282

Sarfati J, Guiochon-Mantel A, Rondard P, Arnulf I, Garcia-Piñero A, Wolczynski S, Brailly-Tabard S, Bidet M, Ramos-Arroyo M, Mathieu M, Lienhardt-Roussie A, Morgan G, Turki Z, Bremont C, Lespinasse J, Du Boullay H, Chabbert-Buffet N, Jacquemont $\mathrm{S}$, Reach G, De Talence N, Tonella P, Conrad B, Despert F, Delobel B, Brue T, Bouvattier C, Cabrol S, Pugeat M, Murat A, Bouchard P, Hardelin JP, Dodé C, Young J (2010) A comparative phenotypic study of Kallmann syndrome patients carrying monoallelic and biallelic mutations in the prokineticin 2 or prokineticin receptor 2 genes. J Clin Endocrinol Metab 95:659-669

Sato K, Emi M, Ezura Y, Fujita Y, Takada D, Ishigami T, Umemura S, Xin Y, Wu LL, Larrinaga-Shum S, Stephenson SH, Hunt SC, Hopkins PN (2004) Soluble epoxide hydrolase variant (Glu287Arg) modifies plasma total cholesterol and triglyceride phenotype in familial hypercholesterolemia: intrafamilial association study in an eight-generation hyperlipidemic kindred. J Hum Genet 49:29-34

Sato D, Lionel AC, Leblond CS, Prasad A, Pinto D, Walker S, O'Connor I, Russell C, Drmic IE, Hamdan FF, Michaud JL, Endris V, Roeth R, Delorme R, Huguet G, Leboyer M, Rastam M, Gillberg C, Lathrop M, Stavropoulos DJ, Anagnostou E, Weksberg R, Fombonne E, Zwaigenbaum L, Fernandez BA, Roberts W, Rappold GA, Marshall CR, Bourgeron T, Szatmari P, Scherer SW (2012) SHANK1 deletions in males with autism spectrum disorder. Am J Hum Genet 90:879-887

Savage DB, Agostini M, Barroso I, Gurnell M, Luan J, Meirhaeghe A, Harding AH, Ihrke G, Rajanayagam O, Soos MA, George S, Berger D, Thomas EL, Bell JD, Meeran K, Ross RJ, Vidal-Puig A, Wareham NJ, O'Rahilly S, Chatterjee VK, Schafer AJ (2002) Digenic inheritance of severe insulin resistance in a human pedigree. Nat Genet 31:379-384

Schaaf CP, Blazo M, Lewis RA, Tonini RE, Takei H, Wang J, Wong LJ, Scaglia F (2011a) Early-onset severe neuromuscular phenotype associated with compound heterozygosity for OPAl mutations. Mol Genet Metab 103:383-387

Schaaf CP, Sabo A, Sakai Y, Crosby J, Muzny D, Hawes A, Lewis L, Akbar H, Varghese R, Boerwinkle E, Gibbs RA, Zoghbi HY (2011b) Oligogenic heterozygosity in individuals with highfunctioning autism spectrum disorders. Hum Mol Genet 20:3366-3375

Scheffer H, Van Der Vlies P, Burton M, Verlind E, Moll AC, Imhof SM, Buys CH (2000) Two novel germline mutations of the retinoblastoma gene $(R B 1)$ that show incomplete penetrance, one splice site and one missense. J Med Genet 37:E6

Scherer D, Kumar R (2010) Genetics of pigmentation in skin cancer-a review. Mutat Res 705:141-153 
Schild R, Knüppel T, Konrad M, Bergmann C, Trautmann A, Kemper MJ, Wu K, Yaklichkin S, Wang J, Pestell R, Müller-Wiefel DE, Schaefer F, Weber S (2013) Double homozygous missense mutations in $D A C H 1$ and $B M P 4$ in a patient with bilateral cystic renal dysplasia. Nephrol Dial Transplant 28:227-232

Schneider A, Larusch J, Sun X, Aloe A, Lamb J, Hawes R, Cotton P, Brand RE, Anderson MA, Money ME, Banks PA, Lewis MD, Baillie J, Sherman S, Disario J, Burton FR, Gardner TB, Amann ST, Gelrud A, George R, Rockacy MJ, Kassabian S, Martinson J, Slivka A, Yadav D, Oruc N, Barmada MM, Frizzell R, Whitcomb DC (2011) Combined bicarbonate conductanceimpairing variants in CFTR and SPINK1 variants are associated with chronic pancreatitis in patients without cystic fibrosis. Gastroenterology 140:162-171

Schork NJ, Murray SS, Frazer KA, Topol EJ (2009) Common vs. rare allele hypotheses for complex diseases. Curr Opin Genet Dev 19:212-219

Schubert EL, Strong LC, Hansen MF (1997) A splicing mutation in $R B 1$ in low penetrance retinoblastoma. Hum Genet 100:557-563

Schwartz PJ, Priori SG, Napolitano C (2003) How really rare are rare diseases?: the intriguing case of independent compound mutations in the long QT syndrome. J Cardiovasc Electrophysiol 14:1120-1121

Sequeiros J, Ramos EM, Cerqueira J, Costa MC, Sousa A, PintoBasto J, Alonso I (2010) Large normal and reduced penetrance alleles in Huntington disease: instability in families and frequency at the laboratory, at the clinic and in the population. Clin Genet 78:381-387

Serrano-Fernández P, Debniak T, Górski B, Bogdanova N, Dörk T, Cybulski C, Huzarski T, Byrski T, Gronwald J, Wokołorczyk D, Narod SA, Lubiński J (2009) Synergistic interaction of variants in $C H E K 2$ and $B R C A 2$ on breast cancer risk. Breast Cancer Res Treat 117:161-165

Sévin M, Kutalik Z, Bergman S, Vercelletto M, Renou P, Lamy E, Vingerhoets FJ, Di Virgilio G, Boisseau P, Bezieau S, Pasquier L, Rival JM, Beckmann JS, Damier P, Jacquemont S (2009) Penetrance of marked cognitive impairment in older male carriers of the FMRl gene premutation. J Med Genet 46:818-824

Shalata A, Furman H, Adir V, Adir N, Hujeirat Y, Shalev SA, Borochowitz ZU (2010) Myotonia congenita in a large consanguineous Arab family: insight into the clinical spectrum of carriers and double heterozygotes of a novel mutation in the chloride channel CLCN1 gene. Muscle Nerve 41:464-469

Shankar RK, Ellard S, Standiford D, Pihoker C, Gilliam LK, Hattersley A, Dolan LM (2013) Digenic heterozygous HNF1A and HNF4A mutations in two siblings with childhood-onset diabetes. Pediatr Diabetes (in press)

Shaw ND, Seminara SB, Welt CK, Au MG, Plummer L, Hughes VA, Dwyer AA, Martin KA, Quinton R, Mericq V, Merino PM, Gusella JF, Crowley WF Jr, Pitteloud N, Hall JE (2011) Expanding the phenotype and genotype of female GnRH deficiency. J Clin Endocrinol Metab 96:E566-E576

Shen J (2009) Evaluation of environmental and personal susceptibility characteristics that modify genetic risks. Methods Mol Biol 471:163-177

Shen H, Li J, Zhang J, Xu C, Jiang Y, Wu Z, Zhao F, Liao L, Chen J, Lin Y, Tian Q, Papasian CJ, Deng H-W (2013a) Comprehensive characterization of human genome variation by high coverage whole-genome sequencing of forty four Caucasians. PLoS ONE 8:e59494

Shen W, Gu Y, Zhu R, Zhang L, Zhang J, Ying C (2013b) Copy number variations of the $F 8$ gene are associated with venous thromboembolism. Blood Cells Mol Dis 50:259-262

Shinlapawittayatorn K, Du XX, Liu H, Ficker E, Kaufman ES, Deschênes I (2011) A common SCN5A polymorphism modulates the biophysical defects of SCN5A mutations. Heart Rhythm 8:455-462

Shirahama S, Miyahara A, Kitoh H, Honda A, Kawase A, Yamada K, Mabuchi A, Kura H, Yokoyama Y, Tsutsumi M, Ikeda T, Tanaka N, Nishimura G, Ohashi H, Ikegawa S (2003) Skewed $\mathrm{X}$-chromosome inactivation causes intra-familial phenotypic variation of an EBP mutation in a family with $\mathrm{X}$-linked dominant chondrodysplasia punctata. Hum Genet 112:78-83

Shohat M, Halpern GJ (2011) Familial Mediterranean fever-a review. Genet Med 13:487-498

Sibille A, Eng CM, Kim SJ, Pastores G, Grabowski GA (1993) Phenotype/genotype correlations in Gaucher disease type I: clinical and therapeutic implications. Am J Hum Genet 52:1094-1101

Sidransky E (2006) Heterozygosity for a Mendelian disorder as a risk factor for complex disease. Clin Genet 70:275-282

Siegert S, Hampe J, Schafmayer C, von Schönfels W, Egberts JH, Försti A, Chen B, Lascorz J, Hemminki K, Franke A, Nothnagel M, Nöthlings U, Krawczak M (2013) Genome-wide investigation of gene-environment interactions in colorectal cancer. Hum Genet 132:219-231

Sierra M, González-Aramburu I, Sánchez-Juan P, Sánchez-Quintana C, Polo JM, Berciano J, Combarros O, Infante J (2011) High frequency and reduced penetrance of LRRK2 G2019S mutation among Parkinson's disease patients in Cantabria (Spain). Mov Disord 26:2343-2346

Silva E, Dharmaraj S, Li YY, Pina AL, Carter RC, Loyer M, Traboulsi E, Theodossiadis G, Koenekoop R, Sundin O, Maumenee I (2004) A missense mutation in $G U C Y 2 D$ acts as a genetic modifier in RPE65-related Leber Congenital Amaurosis. Ophthalmic Genet 25:205-217

Simi L, Sestini R, Ferruzzi P, Gaglianò MS, Gensini F, Mascalchi M, Guerrini L, Pratesi C, Pinzani P, Nesi G, Ercolino T, Genuardi M, Mannelli M (2005) Phenotype variability of neural crest derived tumours in six Italian families segregating the same founder SDHD mutation Q109X. J Med Genet 42:e52

Singh NA, Pappas C, Dahle EJ, Claes LR, Pruess TH, De Jonghe P, Thompson J, Dixon M, Gurnett C, Peiffer A, White HS, Filloux F, Leppert MF (2009) A role of $S C N 9 A$ in human epilepsies, as a cause of febrile seizures and as a potential modifier of Dravet syndrome. PLoS Genet 5:e1000649

Skelly DA, Ronald J, Akey JM (2009) Inherited variation in gene expression. Annu Rev Genomics Hum Genet 10:313-332

Sleegers K, Bettens K, Brouwers N, Engelborghs S, van Miegroet H, De Deyn PP, Van Broeckhoven C (2009) Common variation in GRB-associated binding protein $2(G A B 2)$ and increased risk for Alzheimer dementia. Hum Mutat 30:E338-E344

Smaoui N, Chaabouni M, Sergeev YV, Kallel H, Li S, Mahfoudh N, Maazoul F, Kammoun H, Gandoura N, Bouaziz A, Nouiri E, M'Rad R, Chaabouni H, Hejtmancik JF (2006) Screening of the eight BBS genes in Tunisian families: no evidence of triallelism. Invest Ophthalmol Vis Sci 47(8):3487-3495

Smith MW, Dean M, Carrington M, Winkler C, Huttley GA, Lomb DA, Goedert JJ, O'Brien TR, Jacobson LP, Kaslow R, Buchbinder S, Vittinghoff E, Vlahov D, Hoots K, Hilgartner MW, O'Brien SJ (1997) Contrasting genetic influence of CCR2 and CCR5 variants on HIV-1 infection and disease progression. Hemophilia Growth and Development Study (HGDS), Multicenter AIDS Cohort Study (MACS), Multicenter Hemophilia Cohort Study (MHCS), San Francisco City Cohort (SFCC), ALIVE Study. Science 277:959-965

Snozek CL, Lagerstedt SA, Khoo TK, Rubenfire M, Isley WL, Train LJ, Baudhuin LM (2009) $L D L R$ promoter variant and exon 14 mutation on the same chromosome are associated with an unusually severe FH phenotype and treatment resistance. Eur J Hum Genet 17:85-90 
Soares ML, Coelho T, Sousa A, Batalov S, Conceição I, Sales-Luís ML, Ritchie MD, Williams SM, Nievergelt CM, Schork NJ, Saraiva MJ, Buxbaum JN (2005) Susceptibility and modifier genes in Portuguese transthyretin V30M amyloid polyneuropathy: complexity in a single-gene disease. Hum Mol Genet 14:543-553

Song MJ, Lee ST, Lee MK, Ji Y, Kim JW, Ki CS (2012) Estimation of carrier frequencies of six autosomal-recessive Mendelian disorders in the Korean population. J Hum Genet 57:139-144

Soriano A, Manna R (2012) Familial Mediterranean fever: new phenotypes. Autoimmun Rev 12:31-37

Soto-Ramírez N, Arshad SH, Holloway JW, Zhang H, Schauberger E, Ewart S, Patil V, Karmaus W (2013) The interaction of genetic variants and DNA methylation of the interleukin-4 receptor gene increase the risk of asthma at age 18 years. Clin Epigenetics 5:1

Sparrow DB, Chapman G, Smith AJ, Mattar MZ, Major JA, O'Reilly VC, Saga Y, Zackai EH, Dormans JP, Alman BA, McGregor L, Kageyama R, Kusumi K, Dunwoodie SL (2012) A mechanism for gene-environment interaction in the etiology of congenital scoliosis. Cell 149:295-306

Spurdle AB, Whiley PJ, Thompson B, Feng B, Healey S, Brown MA, Pettigrew C; kConFab, Van Asperen CJ, Ausems MG, Kattentidt-Mouravieva AA, van den Ouweland AM; Dutch Belgium UV Consortium, Lindblom A, Pigg MH, Schmutzler RK, Engel C, Meindl A; German Consortium of Hereditary Breast and Ovarian Cancer, Caputo S, Sinilnikova OM, Lidereau R; French COVAR group collaborators, Couch FJ, Guidugli L, Hansen Tv, Thomassen M, Eccles DM, Tucker K, Benitez J, Domchek SM, Toland AE, Van Rensburg EJ, Wappenschmidt B, Borg A, Vreeswijk MP, Goldgar DE; ENIGMA Consortium (2012) BRCA1 R1699Q variant displaying ambiguous functional abrogation confers intermediate breast and ovarian cancer risk. J Med Genet 49:525-532

Staropoli JF, Xin W, Barone R, Cotman SL, Sims KB (2012) An atypical case of neuronal ceroid lipofuscinosis with co-inheritance of a variably penetrant $P O L G 1$ mutation. BMC Med Genet 13:50

Steinberg MH, Sebastiani P (2012) Genetic modifiers of sickle cell disease. Am J Hematol 87:795-803

Stenson PD, Mort M, Ball EV, Howells K, Phillips AD, Thomas NS, Cooper DN (2009) The human gene mutation database: 2008 update. Genome Med 1:13

Stranger BE, Nica AC, Forrest MS, Dimas A, Bird CP, Beazley C, Ingle CE, Dunning M, Flicek P, Koller D, Montgomery S, Tavaré S, Deloukas P, Dermitzakis ET (2007) Population genomics of human gene expression. Nat Genet 39:1217-1224

Striano P, Weber YG, Toliat MR, Schubert J, Leu C, Chaimana R, Baulac S, Guerrero R, LeGuern E, Lehesjoki AE, Polvi A, Robbiano A, Serratosa JM, Guerrini R, Nürnberg P, Sander T, Zara F, Lerche H, Marini C, EPICURE Consortium (2012) GLUT1 mutations are a rare cause of familial idiopathic generalized epilepsy. Neurology 78:557-562

Strom SP, Gorin MB (2013) Evaluation of autosomal dominant retinal dystrophy genes in an unaffected cohort suggests rare or private missense variants may often be benign. Mol Vis 19:980-985

Stutzmann F, Tan K, Vatin V, Dina C, Jouret B, Tichet J, Balkau B, Potoczna N, Horber F, O'Rahilly S, Farooqi IS, Froguel P, Meyre D (2008) Prevalence of melanocortin-4 receptor deficiency in Europeans and their age-dependent penetrance in multigenerational pedigrees. Diabetes 57:2511-2518

Subramanian S (2012) Quantifying harmful mutations in human populations. Eur J Hum Genet 20:1320-1322

Sugawara H, Iwamoto K, Bundo M, Ueda J, Miyauchi T, Komori A, Kazuno A, Adati N, Kusumi I, Okazaki Y, Ishigooka J, Kojima T, Kato T (2011) Hypermethylation of serotonin transporter gene in bipolar disorder detected by epigenome analysis of discordant monozygotic twins. Transl Psychiatry 1:e24

Sullivan LS, Bowne SJ, Birch DG, Hughbanks-Wheaton D, Heckenlively JR, Lewis RA, Garcia CA, Ruiz RS, Blanton SH, Northrup H, Gire AI, Seaman R, Duzkale H, Spellicy CJ, Zhu J, Shankar SP, Daiger SP (2006) Prevalence of disease-causing mutations in families with autosomal dominant retinitis pigmentosa: a screen of known genes in 200 families. Invest Ophthalmol Vis Sci 47:3052-3064

Sullivan M, Rybicki LA, Winter A, Hoffmann MM, Reiermann S, Linke H, Arbeiter K, Patzer L, Budde K, Hoppe B, Zeier M, Lhotta K, Bock A, Wiech T, Gaspert A, Fehr T, Woznowski M, Berisha G, Malinoc A, Goek ON, Eng C, Neumann HP (2011) Age-related penetrance of hereditary atypical hemolytic uremic syndrome. Ann Hum Genet 75:639-647

Svenson IK, Ashley-Koch AE, Gaskell PC, Riney TJ, Cumming WJ, Kingston HM, Hogan EL, Boustany RM, Vance JM, Nance MA, Pericak-Vance MA, Marchuk DA (2001) Identification and expression analysis of spastin gene mutations in hereditary spastic paraplegia. Am J Hum Genet 68:1077-1085

Sykiotis GP, Plummer L, Hughes VA, Au M, Durrani S, NayakYoung S, Dwyer AA, Quinton R, Hall JE, Gusella JF, Seminara SB, Crowley WF Jr, Pitteloud N (2010) Oligogenic basis of isolated gonadotropin-releasing hormone deficiency. Proc Natl Acad Sci USA 107:15140-15144

Synofzik M, Ronchi D, Keskin I, Basak AN, Wilhelm C, Gobbi C, Birve A, Biskup S, Zecca C, Fernández-Santiago R, Kaugesaar T, Schöls L, Marklund SL, Andersen PM (2012) Mutant superoxide dismutase-1 indistinguishable from wild-type causes ALS. Hum Mol Genet 21:3568-3574

Szabó A, Sahin-Tóth M (2012) Increased activation of hereditary pancreatitis-associated human cationic trypsinogen mutants in presence of chymotrypsin C. J Biol Chem 287:20701-20710

Szyf M (2012) How do environments talk to genes? Nat Neurosci $16: 2-4$

Szymanski M, Wang R, Bassett SS, Avramopoulos D (2011) Alzheimer's risk variants in the clusterin gene are associated with alternative splicing. Transl Psychiatry 1:e18

Tada H, Kawashiri MA, Ohtani R, Noguchi T, Nakanishi C, Konno T, Hayashi K, Nohara A, Inazu A, Kobayashi J, Mabuchi H, Yamagishi M (2011) A novel type of familial hypercholesterolemia: double heterozygous mutations in LDL receptor and LDL receptor adaptor protein 1 gene. Atherosclerosis 219:663-666

Taddei TH, Kacena KA, Yang M, Yang R, Malhotra A, Boxer M, Aleck KA, Rennert G, Pastores GM, Mistry PK (2009) The underrecognized progressive nature of N370S Gaucher disease and assessment of cancer risk in 403 patients. Am J Hematol $84: 208-214$

Tait RC, Walker ID, Perry DJ, Islam SI, Daly ME, McCall F, Conkie JA, Carrell RW (1994) Prevalence of antithrombin deficiency in the healthy population. Br J Haematol 87:106-112

Tait RC, Walker ID, Reitsma PH, Islam SI, McCall F, Poort SR, Conkie JA, Bertina RM (1995) Prevalence of protein C deficiency in the healthy population. Thromb Haemost 73:87-93

Takada D, Ezura Y, Ono S, Iino Y, Katayama Y, Xin Y, Wu LL, Larringa-Shum S, Stephenson SH, Hunt SC, Hopkins PN, Emi M (2003a) Apolipoprotein $\mathrm{H}$ variant modifies plasma triglyceride phenotype in familial hypercholesterolemia: a molecular study in an eight-generation hyperlipidemic family. $\mathrm{J}$ Atheroscler Thromb 10:79-84

Takada D, Ezura Y, Ono S, Iino Y, Katayama Y, Xin Y, Wu LL, Larringa-Shum S, Stephenson SH, Hunt SC, Hopkins PN, Emi M (2003b) Growth hormone receptor variant (L526I) modifies plasma HDL cholesterol phenotype in familial hypercholesterolemia: intra-familial association study in an eight-generation hyperlipidemic kindred. Am J Med Genet 121A:136-140 
Talmud PJ (2007) Rare APOA5 mutations-clinical consequences, metabolic and functional effects: an ENID review. Atherosclerosis 194:287-292

Tamanaha R, Camacho CP, Pereira AC, da Silva AM, Maciel RM, Cerutti JM (2009) Evaluation of RET polymorphisms in a sixgeneration family with G533C RET mutation: specific RET variants may modulate age at onset and clinical presentation. Clin Endocrinol 71:56-64

Tang B, Xiong H, Sun P, Zhang Y, Wang D, Hu Z, Zhu Z, Ma H, Pan Q, Xia JH, Xia K, Zhang Z (2006) Association of PINK1 and $D J-1$ confers digenic inheritance of early onset Parkinson's disease. Hum Mol Genet 15:1816-1825

Tang CS, Sribudiani Y, Miao XP, de Vries AR, Burzynski G, So MT, Leon YY, Yip BH, Osinga J, Hui KJ, Verheij JB, Cherny SS, Tam PK, Sham PC, Hofstra RM, Garcia-Barceló MM (2010) Fine mapping of the 9q31 Hirschsprung's disease locus. Hum Genet 127:675-683

Tang CS, Tang WK, So MT, Miao XP, Leung BM, Yip BH, Leon TY, Ngan ES, Lui VC, Chen Y, Chan IH, Chung PH, Liu XL, Wu XZ, Wong KK, Sham PC, Cherny SS, Tam PK, GarciaBarceló MM (2011) Fine mapping of the NRG1 Hirschsprung's disease locus. PLoS ONE 6:e16181

Tang CS, Ngan ES, Tang WK, So MT, Cheng G, Miao XP, Leon TY, Leung BM, Hui KJ, Lui VH, Chen Y, Chan IH, Chung PH, Liu XL, Wong KK, Sham PC, Cherny SS, Tam PK, Garcia-Barcelo MM (2012a) Mutations in the NRG1 gene are associated with Hirschsprung disease. Hum Genet 131:67-76

Tang CS, Cheng G, So MT, Yip BH, Miao XP, Wong EH, Ngan ES, Lui VC, Song YQ, Chan D, Cheung K, Yuan ZW, Lei L, Chung PH, Liu XL, Wong KK, Marshall CR, Scherer SW, Cherny SS, Sham PC, Tam PK, Garcia-Barceló MM (2012b) Genome-wide copy number analysis uncovers a new HSCR gene: NRG3. PLoS Genet 8:e1002687

Tavtigian SV, Greenblatt MS, Lesueur F, Byrnes GB, IARC Unclassified Genetic Variants Working Group (2008) In silico analysis of missense substitutions using sequence-alignment based methods. Hum Mutat 29:1327-1336

Taylor M, Dehainault C, Desjardins L, Doz F, Levy C, Sastre X, Couturier J, Stoppa-Lyonnet D, Houdayer C, Gauthier-Villars M (2007) Genotype-phenotype correlations in hereditary familial retinoblastoma. Hum Mutat 28:284-293

Taylor A, Bayly G, Patel K, Yarram L, Williams M, Hamilton-Shield J, Humphries SE, Norbury G (2010) A double heterozygote for familial hypercholesterolaemia and familial defective apolipoprotein B-100. Ann Clin Biochem 47:487-490

Tennessen JA, Bigham AW, O'Connor TD, Fu W, Kenny EE, Gravel S, McGee S, Do R, Liu X, Jun G, Kang HM, Jordan D, Leal SM, Gabriel S, Rieder MJ, Abecasis G, Altshuler D, Nickerson DA, Boerwinkle E, Sunyaev S, Bustamante CD, Bamshad MJ, Akey JM, Broad GO, Seattle GO, on behalf of the NHLBI Exome Sequencing Project (2012) Evolution and functional impact of rare coding variation from deep sequencing of human exomes. Science 337:64-69

Tester DJ, Will ML, Haglund CM, Ackerman MJ (2005) Compendium of cardiac channel mutations in 541 consecutive unrelated patients referred for long QT syndrome genetic testing. Heart Rhythm 2:507-517

Thauvin-Robinet C, Munck A, Huet F, Génin E, Bellis G, Gautier E, Audrézet MP, Férec C, Lalau G, Georges MD, Claustres M, Bienvenu T, Gérard B, Boisseau P, Cabet-Bey F, Feldmann D, Clavel C, Bieth E, Iron A, Simon-Bouy B, Costa C, Medina R, Leclerc J, Hubert D, Nové-Josserand R, Sermet-Gaudelus I, Rault G, Flori J, Leroy S, Wizla N, Bellon G, Haloun A, PerezMartin S, d'Acremont G, Corvol H, Clément A, Houssin E, Binquet C, Bonithon-Kopp C, Alberti-Boulmé C, Morris MA, Faivre L, Goossens M, Roussey M, Collaborating Working
Group on R117H, Girodon E (2009) The very low penetrance of cystic fibrosis for the $\mathrm{R} 117 \mathrm{H}$ mutation: a reappraisal for genetic counselling and newborn screening. J Med Genet 46:752-758

The 1000 Genomes Project Consortium (2010) A map of human genome variation from population-scale sequencing. Nature 467:1061-1073

Thiadens AA, Roosing S, Collin RW, van Moll-Ramirez N, van LithVerhoeven JJ, van Schooneveld MJ, den Hollander AI, van den Born LI, Hoyng CB, Cremers FP, Klaver CC (2010) Comprehensive analysis of the achromatopsia genes CNGA3 and $C N G B 3$ in progressive cone dystrophy. Ophthalmology 117:825-830

Thiel C, Kessler K, Giessl A, Dimmler A, Shalev SA, von der Haar S, Zenker M, Zahnleiter D, Stöss H, Beinder E, Abou Jamra R, Ekici AB, Schröder-Kress N, Aigner T, Kirchner T, Reis A, Brandstätter JH, Rauch A (2011) NEK1 mutations cause shortrib polydactyly syndrome type Majewski. Am J Hum Genet $88: 106-114$

Thorgeirsson TE, Gudbjartsson DF, Surakka I, Vink JM, Amin N, Geller F, Sulem P, Rafnar T, Esko T, Walter S, Gieger C, Rawal R, Mangino M, Prokopenko I, Mägi R, Keskitalo K, Gudjonsdottir IH, Gretarsdottir S, Stefansson H, Thompson JR, Aulchenko YS, Nelis M, Aben KK, den Heijer M, Dirksen A, Ashraf H, Soranzo N, Valdes AM, Steves C, Uitterlinden AG, Hofman A, Tönjes A, Kovacs P, Hottenga JJ, Willemsen G, Vogelzangs N, Döring A, Dahmen N, Nitz B, Pergadia ML, Saez B, De Diego V, Lezcano V, Garcia-Prats MD, Ripatti S, Perola M, Kettunen J, Hartikainen AL, Pouta A, Laitinen J, Isohanni M, Huei-Yi S, Allen M, Krestyaninova M, Hall AS, Jones GT, van Rij AM, Mueller T, Dieplinger B, Haltmayer M, Jonsson S, Matthiasson SE, Oskarsson H, Tyrfingsson T, Kiemeney LA, Mayordomo JI, Lindholt JS, Pedersen JH, Franklin WA, Wolf H, Montgomery GW, Heath AC, Martin NG, Madden PA, Giegling I, Rujescu D, Järvelin MR, Salomaa V, Stumvoll M, Spector TD, Wichmann HE, Metspalu A, Samani NJ, Penninx BW, Oostra BA, Boomsma DI, Tiemeier H, van Duijn CM, Kaprio J, Gulcher JR, ENGAGE Consortium, McCarthy MI, Peltonen L, Thorsteinsdottir U, Stefansson K (2008) Sequence variants at CHRNB3-CHRNA6 and CYP2A6 affect smoking behavior. Nat Genet 42:448-453

Tirado I, Mateo J, Soria JM, Oliver A, Borrell M, Coll I, Vallvé C, Souto JC, Martínez-Sánchez E, Fontcuberta J (2001) Contribution of prothrombin 20210A allele and factor V Leiden mutation to thrombosis risk in thrombophilic families with other hemostatic deficiencies. Haematologica 86:1200-1208

Tirado I, Mateo J, Soria JM, Oliver A, Martínez-Sánchez E, Vallvé C, Borrell M, Urrutia T, Fontcuberta J (2005) The ABO blood group genotype and factor VIII levels as independent risk factors for venous thromboembolism. Thromb Haemost 93:468-474

Titeux M, Pendaries V, Tonasso L, Décha A, Bodemer C, Hovnanian A (2008) A frequent functional SNP in the $M M P 1$ promoter is associated with higher disease severity in recessive dystrophic epidermolysis bullosa. Hum Mutat 29:267-276

To-Figueras J, Ducamp S, Clayton J, Badenas C, Delaby C, Ged C, Lyoumi S, Gouya L, de Verneuil H, Beaumont C, Ferreira GC, Deybach JC, Herrero C, Puy H (2011) ALAS2 acts as a modifier gene in patients with congenital erythropoietic porphyria. Blood 118:1443-1451

Toledo RA, Wagner SM, Coutinho FL, Lourenço DM Jr, Azevedo JA, Longuini VC, Reis MT, Siqueira SA, Lucon AM, Tavares MR, Fragoso MC, Pereira AA, Dahia PL, Mulligan LM, Toledo SP (2010) High penetrance of pheochromocytoma associated with the novel C634Y/Y791F double germline mutation in the RET protooncogene. J Clin Endocrinol Metab 95:1318-1327

Tonooka N, Tomura H, Takahashi Y, Onigata K, Kikuchi N, Horikawa Y, Mori M, Takeda J (2002) High frequency of 
mutations in the HNF-1 $\alpha$ gene in non-obese patients with diabetes of youth in Japanese and identification of a case of digenic inheritance. Diabetologia 45:1709-1712

Tory K, Lacoste T, Burglen L, Morinière V, Boddaert N, Macher MA, Llanas B, Nivet H, Bensman A, Niaudet P, Antignac C, Salomon $\mathrm{R}$, Saunier S (2007) High NPHP1 and NPHP6 mutation rate in patients with Joubert syndrome and nephronophthisis: potential epistatic effect of NPHP6 and AHII mutations in patients with NPHP1 mutations. J Am Soc Nephrol 18:1566-1575

Touitou I, Perez C, Dumont B, Federici L, Jorgensen C (2006) Refractory auto-inflammatory syndrome associated with digenic transmission of low-penetrance tumour necrosis factor receptorassociated periodic syndrome and cryopyrin-associated periodic syndrome mutations. Ann Rheum Dis 65:1530-1531

Tropeano M, Ahn JW, Dobson RJ, Breen G, Rucker J, Dixit A, Pal DK, McGuffin P, Farmer A, White PS, Andrieux J, Vassos E, Ogilvie CM, Curran S, Collier DA (2013) Male-biased autosomal effect of 16p13.11 copy number variation in neurodevelopmental disorders. PLoS ONE 8:e61365

Tzetis M, Kaliakatsos M, Fotoulaki M, Papatheodorou A, Doudounakis S, Tsezou A, Makrythanasis P, Kanavakis E, NousiaArvanitakis S (2007) Contribution of the CFTR gene, the pancreatic secretory trypsin inhibitor gene (SPINK1) and the cationic trypsinogen gene (PRSS1) to the etiology of recurrent pancreatitis. Clin Genet 71:451-457

Uhrhammer N, Bignon YJ (2008) Report of a family segregating mutations in both the $A P C$ and $M S H 2$ genes: juvenile onset of colorectal cancer in a double heterozygote. Int J Colorectal Dis 23:1131-1135

Utz VM, Beight CD, Marino MJ, Hagstrom SA, Traboulsi EI. (2013) Autosomal dominant retinitis pigmentosa secondary to premRNA splicing-factor gene PRPF31 (RP11): review of disease mechanism and report of a family with a novel 3-base pair insertion. Ophthalmic Genet (in press)

Vaags AK, Lionel AC, Sato D, Goodenberger M, Stein QP, Curran S, Ogilvie C, Ahn JW, Drmic I, Senman L, Chrysler C, Thompson A, Russell C, Prasad A, Walker S, Pinto D, Marshall CR, Stavropoulos DJ, Zwaigenbaum L, Fernandez BA, Fombonne E, Bolton PF, Collier DA, Hodge JC, Roberts W, Szatmari P, Scherer SW (2012) Rare deletions at the neurexin 3 locus in autism spectrum disorder. Am J Hum Genet 90:133-141

Valenti L, Fracanzani AL, Rametta R, Fraquelli M, Soverini G, Pelusi S, Dongiovanni P, Conte D, Fargion S (2012) Effect of the A736V TMPRSS6 polymorphism on the penetrance and clinical expression of hereditary hemochromatosis. J Hepatol 57:1319-1325

Valle L, Serena-Acedo T, Liyanarachchi S, Hampel H, Comeras I, Li Z, Zeng Q, Zhang HT, Pennison MJ, Sadim M, Pasche B, Tanner SM, de la Chapelle A (2008) Germline allele-specific expression of TGFBR1 confers an increased risk of colorectal cancer. Science 321:1361-1365

Valverde JR, Alonso J, Palacios I, Pestaña A (2005) RB1 gene mutation up-date, a meta-analysis based on 932 reported mutations available in a searchable database. BMC Genet 6:53

van Blitterswijk M, van Es MA, Hennekam EA, Dooijes D, van Rheenen W, Medic J, Bourque PR, Schelhaas HJ, van der Kooi AJ, de Visser M, de Bakker PI, Veldink JH, van den Berg LH (2012a) Evidence for an oligogenic basis of amyotrophic lateral sclerosis. Hum Mol Genet 21:3776-3784

van Blitterswijk M, van Es MA, Koppers M, van Rheenen W, Medic J, Schelhaas HJ, van der Kooi AJ, de Visser M, Veldink JH, van den Berg LH (2012b) VAPB and C9orf72 mutations in 1 familial amyotrophic lateral sclerosis patient. Neurobiol Aging 33:2950.e1-4

van Boven HH, Reitsma PH, Rosendaal FR, Bayston TA, Chowdhury V, Bauer KA, Scharrer I, Conard J, Lane DA (1996) Factor V
Leiden (FV R506Q) in families with inherited antithrombin deficiency. Thromb Haemost 75:417-421

van den Veyver IB (2001) Skewed X inactivation in X-linked disorders. Semin Reprod Med 19:183-191

van der Kolk DM, de Bock GH, Leegte BK, Schaapveld M, Mourits MJ, de Vries J, van der Hout AH, Oosterwijk JC (2010) Penetrance of breast cancer, ovarian cancer and contralateral breast cancer in BRCA1 and BRCA2 families: high cancer incidence at older age. Breast Cancer Res Treat 124:643-651

van der Velden PA, Sandkuijl LA, Bergman W, Pavel S, van Mourik L, Frants RR, Gruis NA (2001) Melanocortin-1 receptor variant R151C modifies melanoma risk in Dutch families with melanoma. Am J Hum Genet 69:774-779

van der Werf C, Nederend I, Hofman N, van Geloven N, Ebink C, Frohn-Mulder IM, Alings AM, Bosker HA, Bracke FA, van den Heuvel F, Waalewijn RA, Bikker H, van Tintelen JP, Bhuiyan ZA, van den Berg MP, Wilde AA (2012) Familial evaluation in catecholaminergic polymorphic ventricular tachycardia: disease penetrance and expression in cardiac ryanodine receptor mutation-carrying relatives. Circ Arrhythm Electrophysiol 5:748-756

Van Driest SL, Vasile VC, Ommen SR, Will ML, Tajik AJ, Gersh BJ, Ackerman MJ (2004) Myosin binding protein C mutations and compound heterozygosity in hypertrophic cardiomyopathy. J Am Coll Cardiol 44:1903-1910

Van Goethem G, Löfgren A, Dermaut B, Ceuterick C, Martin JJ, Van Broeckhoven C (2003) Digenic progressive external ophthalmoplegia in a sporadic patient: recessive mutations in $P O L G$ and C10orf2/Twinkle. Hum Mutat 22:175-176

van Mens TE, Levi M, Middeldorp S (2013) Evolution of Factor V Leiden. Thromb Haemost (in press). doi:10.1160/TH13-02-0115

van Puijenbroek M, Nielsen M, Reinards TH, Weiss MM, Wagner A, Hendriks YM, Vasen HF, Tops CM, Wijnen J, van Wezel T, Hes FJ, Morreau H (2007) The natural history of a combined defect in MSH6 and MUTYH in a HNPCC family. Fam Cancer 6:43-51

van Rijsingen IA, Nannenberg EA, Arbustini E, Elliott PM, Mogensen J, Ast JF, van der Kooi AJ, van Tintelen JP, van den Berg MP, Grasso M, Serio A, Jenkins S, Rowland C, Richard P, Wilde AA, Perrot A, Pankuweit S, Zwinderman AH, Charron P, Christiaans I, Pinto YM (2013) Gender-specific differences in major cardiac events and mortality in lamin $\mathrm{A} / \mathrm{C}$ mutation carriers. Eur J Heart Fail 15:376-384

van Spronsen FJ (2010) Phenylketonuria: a 21st century perspective. Nat. Rev. Endocrinol. 6:509-514

van Tuyll van Serooskerken AM, de Rooij FW, Edixhoven A, Bladergroen RS, Baron JM, Joussen S, Merk HF, Steijlen PM, Poblete-Gutiérrez P, te Velde K, Wilson JH, Koole RH, van Geel M, Frank J (2011) Digenic inheritance of mutations in the coproporphyrinogen oxidase and protoporphyrinogen oxidase genes in a unique type of porphyria. J Invest Dermatol 131:2249-2254

VanderWeele TJ, Asomaning K, Tchetgen Tchetgen EJ, Han Y, Spitz MR, Shete S, Wu X, Gaborieau V, Wang Y, McLaughlin J, Hung RJ, Brennan P, Amos CI, Christiani DC, Lin X (2012) Genetic variants on $15 \mathrm{q} 25.1$, smoking, and lung cancer: an assessment of mediation and interaction. Am $\mathrm{J}$ Epidemiol 175:1013-1020

Vannberg FO, Chapman SJ, Hill AVS (2011) Human genetic susceptibility to intracellular pathogens. Immunol Rev 240:105-116

Varga RE, Schüle R, Fadel H, Valenzuela I, Speziani F, Gonzalez M, Rudenskaia G, Nürnberg G, Thiele H, Altmüller J, Alvarez V, Gamez J, Garbern JY, Nürnberg P, Zuchner S, Beetz C (2013) Don't trust the pedigree: reduced and sex-dependent penetrance at a novel mutation hotspot in ATL1 blurs autosomal dominant inheritance of spastic paraplegia. Hum Mutat 34:860-863 
Vassos E, Collier DA, Holden S, Patch C, Rujescu D, St Clair D, Lewis CM (2010) Penetrance for copy number variants associated with schizophrenia. Hum Mol Genet 19:3477-3481

Venturini G, Rose AM, Shah AZ, Bhattacharya SS, Rivolta C (2012) CNOT3 is a modifier of PRPF31 mutations in retinitis pigmentosa with incomplete penetrance. PLoS Genet 8:e1003040

Vincent AL, Billingsley G, Buys Y, Levin AV, Priston M, Trope G, Williams-Lyn D, Héon E (2002) Digenic inheritance of early onset glaucoma: $C Y P 1 B 1$, a potential modifier gene. Am J Hum Genet 70:448-460

Vital A, Latour P, Sole G, Ferrer X, Rouanet M, Tison F, Vital C, Goizet C (2012) A French family with Charcot-Marie-Tooth disease related to simultaneous heterozygous MFN2 and GDAP1 mutations. Neuromuscul Disord 22:735-741

Vitali T, Sossi V, Tiziano F, Zappata S, Giuli A, Paravatou-Petsotas M, Neri G, Brahe C (1999) Detection of the survival motor neuron (SMN) genes by FISH: further evidence for a role for SMN2 in the modulation of disease severity in SMA patients. Hum Mol Genet 8:2525-2532

Vithana EN, Abu-Safieh L, Pelosini L, Winchester E, Hornan D, Bird AC, Hunt DM, Bustin SA, Bhattacharya SS (2003) Expression of PRPF31 mRNA in patients with autosomal dominant retinitis pigmentosa: a molecular clue for incomplete penetrance? Invest Ophthalmol Vis Sci 44:4204-4209

Vitko I, Chen Y, Arias JM, Shen Y, Wu XR, Perez-Reyes E (2005) Functional characterization and neuronal modeling of the effects of childhood absence epilepsy variants of $C A C N A 1 H$, a T-type calcium channel. J Neurosci 25:4844-4855

Vockley J, Rinaldo P, Bennett MJ, Matern D, Vladutiu GD (2000) Synergistic heterozygosity: disease resulting from multiple partial defects in one or more metabolic pathways. Mol Genet Metab 71:10-18

Vogel F, Motulsky AG (1997) Human genetics. Problems and approaches.s 3rd edn., Springer, Berlin

Vogt J, Kohlhase J, Morlot S, Kluwe L, Mautner VF, Cooper DN, Kehrer-Sawatzki H (2011) Monozygotic twins discordant for neurofibromatosis type 1 due to a postzygotic $N F 1$ gene mutation. Hum Mutat 32:E2134-E2147

Vujic M, Heyer CM, Ars E, Hopp K, Markoff A, Orndal C, Rudenhed B, Nasr SH, Torres VE, Torra R, Bogdanova N, Harris PC (2010) Incompletely penetrant $P K D 1$ alleles mimic the renal manifestations of ARPKD. J Am Soc Nephrol 21:1097-1102

Vytopil M, Ricci E, Dello Russo A, Hanisch F, Neudecker S, Zierz S, Ricotti R, Demay L, Richard P, Wehnert M, Bonne G, Merlini L, Toniolo D (2002) Frequent low penetrance mutations in the lamin A/C gene, causing Emery Dreifuss muscular dystrophy. Neuromuscul Disord 12:958-963

Waalen J, Beutler E (2009) Genetic screening for low-penetrance variants in protein-coding genes. Annu Rev Genomics Hum Genet 10:431-450

Walker LC, Fredericksen ZS, Wang X, Tarrell R, Pankratz VS, Lindor NM, Beesley J, Healey S, Chen X; KConFab investigators, Stoppa-Lyonnet D, Tirapo C, Giraud S, Mazoyer S, Muller D, Fricker JP, Delnatte C; GEMO Study Collaborators, Schmutzler RK, Wappenschmidt B, Engel C, Schönbuchner I, Deissler H, Meindl A, Hogervorst FB, Verheus M, Hooning MJ, van den Ouweland AM, Nelen MR, Ausems MG, Aalfs CM, van Asperen CJ, Devilee P, Gerrits MM, Waisfisz Q; HEBON, Szabo CI; ModSQuaD, Easton DF, Peock S, Cook M, Oliver CT, Frost D, Harrington P, Evans DG, Lalloo F, Eeles R, Izatt L, Chu C, Davidson R, Eccles D, Ong KR, Cook J; EMBRACE, Rebbeck $\mathrm{T}$, Nathanson KL, Domchek SM, Singer CF, GschwantlerKaulich D, Dressler AC, Pfeiler G, Godwin AK, Heikkinen T, Nevanlinna H, Agnarsson BA, Caligo MA, Olsson H, Kristoffersson U, Liljegren A, Arver B, Karlsson P, Melin B; SWEBRCA, Sinilnikova OM, McGuffog L, Antoniou AC, Chenevix-
Trench G, Spurdle AB, Couch FJ (2010) Evidence for SMAD3 as a modifier of breast cancer risk in BRCA2 mutation carriers. Breast Cancer Res 12:R102

Walsh DM, Shalev SA, Simpson MA, Morgan NV, Gelman-Kohan Z, Chemke J, Trembath RC, Maher ER (2013) Acrocallosal syndrome: identification of a novel KIF7 mutation and evidence for oligogenic inheritance. Eur J Med Genet 56:39-42

Walters RG, Jacquemont S, Valsesia A, de Smith AJ, Martinet D, Andersson J, Falchi M, Chen F, Andrieux J, Lobbens S, Delobel B, Stutzmann F, El-Sayed Moustafa JS, Chèvre JC, Lecoeur C, Vatin V, Bouquillon S, Buxton JL, Boute O, Holder-Espinasse M, Cuisset JM, Lemaitre MP, Ambresin AE, Brioschi A, Gaillard M, Giusti V, Fellmann F, Ferrarini A, Hadjikhani N, Campion D, Guilmatre A, Goldenberg A, Calmels N, Mandel JL, Le Caignec C, David A, Isidor B, Cordier MP, Dupuis-Girod S, Labalme A, Sanlaville D, Béri-Dexheimer M, Jonveaux P, Leheup B, Ounap K, Bochukova EG, Henning E, Keogh J, Ellis RJ, Macdermot KD, van Haelst MM, Vincent-Delorme C, Plessis G, Touraine R, Philippe A, Malan V, Mathieu-Dramard M, Chiesa J, Blaumeiser B, Kooy RF, Caiazzo R, Pigeyre M, Balkau B, Sladek R, Bergmann S, Mooser V, Waterworth D, Reymond A, Vollenweider P, Waeber G, Kurg A, Palta P, Esko T, Metspalu A, Nelis M, Elliott P, Hartikainen AL, McCarthy MI, Peltonen L, Carlsson L, Jacobson P, Sjöström L, Huang N, Hurles ME, O'Rahilly S, Farooqi IS, Männik K, Jarvelin MR, Pattou F, Meyre D, Walley AJ, Coin LJ, Blakemore AI, Froguel P, Beckmann JS (2010) A new highly penetrant form of obesity due to deletions on chromosome 16p11.2. Nature 463:671-675

Wang J, Ban MR, Zou GY, Cao H, Lin T, Kennedy BA, Anand S, Yusuf S, Huff MW, Pollex RL, Hegele RA (2008) Polygenic determinants of severe hypertriglyceridemia. Hum Mol Genet 17:2894-2899

Wang X, Pankratz VS, Fredericksen Z, Tarrell R, Karaus M, McGuffog L, Pharaoh PD, Ponder BA, Dunning AM, Peock S, Cook M, Oliver C, Frost D, EMBRACE, Sinilnikova OM, Stoppa-Lyonnet D, Mazoyer S, Houdayer C; GEMO, Hogervorst FB, Hooning MJ, Ligtenberg MJ, HEBON, Spurdle A, Chenevix-Trench G; kConFab, Schmutzler RK, Wappenschmidt B, Engel C, Meindl A, Domchek SM, Nathanson KL, Rebbeck TR, Singer CF, Gschwantler-Kaulich D, Dressler C, Fink A, Szabo CI, Zikan M, Foretova L, Claes K, Thomas G, Hoover RN, Hunter DJ, Chanock SJ, Easton DF, Antoniou AC, Couch FJ (2010a) Common variants associated with breast cancer in genome-wide association studies are modifiers of breast cancer risk in BRCA1 and BRCA2 mutation carriers. Hum Mol Genet 19:2886-2897

Wang J, Spitz MR, Amos CI, Wilkinson AV, Wu X, Shete S (2010b) Mediating effects of smoking and chronic obstructive pulmonary disease on the relation between the CHRNA5-A3 genetic locus and lung cancer risk. Cancer 116:3458-3462

Wang C, Cai Y, Zheng Z, Tang BS, Xu Y, Wang T, Ma J, Chen SD, Langston JW, Tanner CM, Chan P, Chinese Parkinson Study Group (CPSG) (2012) Penetrance of LRRK2 G2385R and $\mathrm{R} 1628 \mathrm{P}$ is modified by common PD-associated genetic variants. Parkinsonism Relat Disord 18:958-963

Wang QY, Song J, Gibbs RA, Boerwinkle E, Dong JF, Yu FL (2013a) Characterizing polymorphisms and allelic diversity of von Willebrand factor gene in the 1,000 Genomes. J Thromb Haemost 11:261-269

Wang Z, Yan A, Lin Y, Xie H, Zhou C, Lan F (2013b) Familial skewed $\mathrm{x}$ chromosome inactivation in adrenoleukodystrophy manifesting heterozygotes from a chinese pedigree. PLoS ONE 8:e57977

Wasielewski M, Riaz M, Vermeulen J, van den Ouweland A, LabrijnMarks I, Olmer R, van der Spaa L, Klijn JG, Meijers-Heijboer H, Dooijes D, Schutte M (2010) Association of rare MSH6 variants 
with familial breast cancer. Breast Cancer Res Treat 123:315-320

Wei AH, Yang XM, Lian S, Li W (2013) Genetic analyses of Chinese patients with digenic oculocutaneous albinism. Chin Med J 126:226-230

Weischenfeldt J, Symmons O, Spitz F, Korbel JO (2013) Phenotypic impact of genomic structural variation: insights from and for human disease. Nat Rev Genet 14:125-138

Weiss G (2010) Genetic mechanisms and modifying factors in hereditary hemochromatosis. Nat Rev Gastroenterol Hepatol 7:50-58

Westenskow P, Splawski I, Timothy KW, Keating MT, Sanguinetti MC (2004) Compound mutations: a common cause of severe long-QT syndrome. Circulation 109:1834-1841

Westra D, Volokhina E, van der Heijden E, Vos A, Huigen M, Jansen J, van Kaauwen E, van der Velden T, van de Kar N, van den Heuvel L (2010) Genetic disorders in complement (regulating) genes in patients with atypical haemolytic uraemic syndrome (aHUS). Nephrol Dial Transplant 25:2195-2202

Westrick RJ, Ginsburg D (2009) Modifier genes for disorders of thrombosis and hemostasis. J Thromb Haemost 7(Suppl. 1):132-135

Wexler NS, Lorimer J, Porter J, Gomez F, Moskowitz C, Shackell E, Marder K, Penchaszadeh G, Roberts SA, Gayán J, Brocklebank D, Cherny SS, Cardon LR, Gray J, Dlouhy SR, Wiktorski S, Hodes ME, Conneally PM, Penney JB, Gusella J, Cha JH, Irizarry M, Rosas D, Hersch S, Hollingsworth Z, MacDonald M, Young AB, Andresen JM, Housman DE, De Young MM, Bonilla E, Stillings T, Negrette A, Snodgrass SR, Martinez-Jaurrieta MD, Ramos-Arroyo MA, Bickham J, Ramos JS, Marshall F, Shoulson I, Rey GJ, Feigin A, Arnheim N, Acevedo-Cruz A, Acosta L, Alvir J, Fischbeck K, Thompson LM, Young A, Dure L, O'Brien CJ, Paulsen J, Brickman A, Krch D, Peery S, Hogarth P, Higgins DS Jr, Landwehrmeyer B, US-Venezuela Collaborative Research Project (2004) Venezuelan kindreds reveal that genetic and environmental factors modulate Huntington's disease age of onset. Proc Natl Acad Sci USA 101:3498-3503

Whitlock EP, Garlitz BA, Harris EL, Beil TL, Smith PR (2006) Screening for hereditary hemochromatosis: systematic review. Ann Intern Med 145:209-223

Wijsman EM, Daw EW, Yu X, Steinbart EJ, Nochlin D, Bird TD, Schellenberg GD (2005) APOE and other loci affect age-at-onset in Alzheimer's disease families with PS2 mutation. Am J Med Genet B Neuropsychiatr Genet 132B:14-20

Williams RB, Chan EK, Cowley MJ, Little PF (2007) The influence of genetic variation on gene expression. Genome Res 17:1707-1716

Williams KL, Fifita JA, Vucic S, Durnall JC, Kiernan MC, Blair IP, Nicholson GA (2013) Pathophysiological insights into ALS with C9ORF72 expansions. J Neurol Neurosurg Psych (in press)

Wilmotte R, Maréchal J, Morlé L, Baklouti F, Philippe N, Kastally R, Kotula L, Delaunay J, Alloisio N (1993) Low expression allele alpha LELY of red cell spectrin is associated with mutations in exon 40 (alpha V/41 polymorphism) and intron 45 and with partial skipping of exon 46. J Clin Invest 91:2091-2096

Wirth B, Brichta L, Schrank B, Lochmüller H, Blick S, Baasner A, Heller R (2006) Mildly affected patients with spinal muscular atrophy are partially protected by an increased SMN2 copy number. Hum Genet 119:422-428

Wolf I, Laitman Y, Rubinek T, Abramovitz L, Novikov I, Beeri R, Kuro-O M, Koeffler HP, Catane R, Freedman LS, Levy-Lahad E, Karlan BY, Friedman E, Kaufman B (2010) Functional variant of KLOTHO: a breast cancer risk modifier among BRCAl mutation carriers of Ashkenazi origin. Oncogene 29:26-33

Wong AH, Gottesman II, Petronis A (2005) Phenotypic differences in genetically identical organisms: the epigenetic perspective. Hum Mol Genet 14 Spec no 1:R11-R18
Xu T, Yang Z, Vatta M, Rampazzo A, Beffagna G, Pilichou K, Scherer SE, Saffitz J, Kravitz J, Zareba W, Danieli GA, Lorenzon A, Nava A, Bauce B, Thiene G, Basso C, Calkins H, Gear K, Marcus F, Towbin JA, Multidisciplinary Study of Right Ventricular Dysplasia Investigators (2010) Compound and digenic heterozygosity contributes to arrhythmogenic right ventricular cardiomyopathy. J Am Coll Cardiol 55:587-597

Xu N, Kim HG, Bhagavath B, Cho SG, Lee JH, Ha K, Meliciani I, Wenzel W, Podolsky RH, Chorich LP, Stackhouse KA, Grove AM, Odom LN, Ozata M, Bick DP, Sherins RJ, Kim SH, Cameron RS, Layman LC (2011) Nasal embryonic LHRH factor (NELF) mutations in patients with normosmic hypogonadotropic hypogonadism and Kallmann syndrome. Fertil Steril 95:1613-1620

Xue Y, Chen Y, Ayub Q, Huang N, Ball EV, Mort M, Phillips AD, Shaw K, Stenson PD, Cooper DN, Tyler-Smith C, the 1000 Genomes Project Consortium (2012) Deleterious- and diseaseallele prevalence in healthy individuals: insights from current predictions, mutation databases, and population-scale resequencing. Am J Hum Genet 91:1022-1032

Yan H, Dobbie Z, Gruber SB, Markowitz S, Romans K, Giardiello FM, Kinzler KW, Vogelstein B (2002) Small changes in expression affect predisposition to tumorigenesis. Nat Genet 30:25-26

Yan X, Zhang T, Wang Z, Jiang Y, Chen Y, Wang H, Ma D, Wang L, $\mathrm{Li} \mathrm{H}$ (2011) A novel mutation in the MITF may be digenic with GJB2 mutations in a large Chinese family of Waardenburg syndrome type II. J Genet Genomics 38:585-591

Yang T, Gurrola JG 2nd, Wu H, Chiu SM, Wangemann P, Snyder PM, Smith RJ (2009) Mutations of KCNJ10 together with mutations of SLC26A4 cause digenic nonsyndromic hearing loss associated with enlarged vestibular aqueduct syndrome. Am J Hum Genet 84:651-657

Yang Z, Wu S, Zheng T, Lu H, Ma X, Jia W, Hu R (2010) Genderdependent penetrance of small heterodimer partner $(S H P)$ gene deficiency in overweight/obese Chinese pedigrees. J Int Med Res 38:142-149

Yang T, Li X, Huang Q, Li L, Chai Y, Sun L, Wang X, Zhu Y, Wang Z, Huang Z, Li Y, Wu H (2013) Double heterozygous mutations of MITF and PAX3 result in Waardenburg syndrome with increased penetrance in pigmentary defects. Clin Genet 83:78-82

Yasuda M, Shabbeer J, Benson SD, Maire I, Burnett RM, Desnick RJ (2003) Fabry disease: characterization of alpha-galactosidase A double mutations and the D313Y plasma enzyme pseudodeficiency allele. Hum Mutat 22:486-492

Yatsenko AN, Shroyer NF, Lewis RA, Lupski JR (2001) Late-onset Stargardt disease is associated with missense mutations that map outside known functional regions of ABCR (ABCA4). Hum Genet 108:346-355

Yigit S, Karakus N, Tasliyurt T, Kaya SU, Bozkurt N, Kisacik B (2012) Significance of $M E F V$ gene R202Q polymorphism in Turkish familial Mediterranean fever patients. Gene 506:43-45

Yoshikane Y, Yoshinaga M, Hamamoto K, Hirose S (2013) A case of long QT syndrome with triple gene abnormalities: digenic mutations in $K C N H 2$ and $S C N 5 A$ and gene variant in KCNE1. Heart Rhythm 10:600-603

Zaghloul NA, Katsanis N (2009) Mechanistic insights into BardetBiedl syndrome, a model ciliopathy. J Clin Invest 119:428-437

Zaghloul NA, Liu Y, Gerdes JM, Gascue C, Oh EC, Leitch CC, Bromberg Y, Binkley J, Leibel RL, Sidow A, Badano JL, Katsanis N (2010) Functional analyses of variants reveal a significant role for dominant negative and common alleles in oligogenic Bardet-Biedl syndrome. Proc Natl Acad Sci USA 107:10602-10607

Zaks N, Shinar Y, Padeh S, Lidar M, Mor A, Tokov I, Pras M, Langevitz P, Pras E, Livneh A (2003) Analysis of the three most 
common $M E F V$ mutations in 412 patients with familial Mediterranean fever. Isr Med Assoc J 5:585-588

Zangen S, Kidron D, Gelbart T, Roy-Chowdhury N, Wang X, Kaplan M (2009) Fatal kernicterus in a girl deficient in glucose-6phosphate dehydrogenase: a paradigm of synergistic heterozygosity. J Pediatr 154:616-619

Zareba W, Moss AJ, Locati EH, Lehmann MH, Peterson DR, Hall WJ, Schwartz PJ, Vincent GM, Priori SG, Benhorin J, Towbin JA, Robinson JL, Andrews ML, Napolitano C, Timothy K, Zhang L, Medina A, International Long QT Syndrome Registry (2003) Modulating effects of age and gender on the clinical course of long QT syndrome by genotype. J Am Coll Cardiol 42:103-109

Zhang W, Bleibel WK, Roe CA, Cox NJ, Dolan ME (2007) Genderspecific differences in expression in human lymphoblastoid cell lines. Pharmacogenet Genomics 17:447-450

Zhang X, Chen S, Zhang L, Liu M, Redfearn S, Bryant RM, Oberti C, Vincent GM, Wang QK (2008) Protective effect of $\mathrm{KCNH} 2$ single nucleotide polymorphism K897T in LQTS families and identification of novel $K C N Q 1$ and $K C N H 2$ mutations. BMC Med Genet 9:87

Zhang CK, Stein PB, Liu J, Wang Z, Yang R, Cho JH, Gregersen PK, Aerts JM, Zhao H, Pastores GM, Mistry PK (2012) Genomewide association study of N370S homozygous Gaucher disease reveals the candidacy of $C L N 8$ gene as a genetic modifier contributing to extreme phenotypic variation. Am J Hematol 87:377-383

Zheng QY, Yan D, Ouyang XM, Du LL, Yu H, Chang B, Johnson KR, Liu XZ (2005) Digenic inheritance of deafness caused by mutations in genes encoding cadherin 23 and protocadherin 15 in mice and humans. Hum Mol Genet 14:103-111

Zimprich A, Grabowski M, Asmus F, Naumann M, Berg D, Bertram M, Scheidtmann K, Kern P, Winkelmann J, Müller-Myhsok B, Riedel L, Bauer M, Müller T, Castro M, Meitinger T, Strom TM, Gasser $T$ (2001) Mutations in the gene encoding epsilonsarcoglycan cause myoclonus-dystonia syndrome. Nat Genet 29:66-69

Zinman L, Liu HN, Sato C, Wakutani Y, Marvelle AF, Moreno D, Morrison KE, Mohlke KL, Bilbao J, Robertson J, Rogaeva E (2009) A mechanism for low penetrance in an ALS family with a novel SOD1 deletion. Neurology 72:1153-1159

Zlotogora J (2003) Penetrance and expressivity in the molecular age. Genet Med 5:347-352

Zlotogora J, van Baal S, Patrinos GP (2007) Documentation of inherited disorders and mutation frequencies in the different religious communities in Israel in the Israeli National Genetic Database. Hum Mutat 28:944-949

Zou Y, Wang J, Liu X, Wang Y, Chen Y, Sun K, Gao S, Zhang C, Wang Z, Zhang Y, Feng X, Song Y, Wu Y, Zhang H, Jia L, Wang H, Wang D, Yan C, Lu M, Zhou X, Song L, Hui R (2013) Multiple gene mutations, not the type of mutation, are the modifier of left ventricle hypertrophy in patients with hypertrophic cardiomyopathy. Mol Biol Rep 40:3969-3976

Zschocke J, Kohlmueller D, Quak E, Meissner T, Hoffmann GF, Mayatepek E (1999) Mild trimethylaminuria caused by common variants in FMO3 gene. Lancet 354:834-835 